\title{
Registration of Images with Varying Topology using Embedded Maps
}

\author{
Xiaoxing Li \\ Dissertation submitted to the Faculty of the \\ Virginia Polytechnic Institute and State University \\ in partial fulfillment of the requirements for the degree of
}

Doctor of Philosophy

in

Computer Engineering
Committee Members:
Christopher Wyatt
Ge Wang
Jason Xuan

A. A. (Louis) Beex

William T. Baumann

Nov. 16, 2010

Blacksburg, VA

Key words: registration, deformable, Riemannian embedding, topological change Copyright 2010, Xiaoxing Li 


\title{
Registration of Images with Varying Topology using Embedded Maps
}

\author{
Xiaoxing Li
}

(ABSTRACT)

In medical images, intensity changes caused by certain pathology can change the topology of image level-sets and are thus commonly referred to as topological changes. Topological changes cause false deformation in existing deformable registration algorithms, which in turn leads to unreliable observations in the clinical study that relies on the deformation fields, such as deformation based morphometry (DBM). In this work, we develop a new deformable registration algorithm for images with topological changes. In our proposed algorithm, 3D images are embedded as 4D surfaces in a Riemannian space. The registration is therefore conducted as a surface evolution, which is modeled by a diffusion process. Our algorithm differs from existing methods in the sense that it takes an a-priori estimation of areas with topological change as an additional input and generates dense deformation vector fields which are free of false deformation. In particular, the output of our algorithm is composed of a diffeomorphic deformation field and an intensity displacement which corrects intensity difference caused by topological changes. By conducting multiple sets of experiments, we demonstrate that our proposed algorithm is capable of accurately registering images with considerable topological changes. More importantly, the resulting deformation field is not impacted by topological changes, i.e., there is no false deformation. 


\section{Dedication}

This work is dedicated to my parents and my dear husband. 


\section{Acknowledgment}

First and foremost, I would like to thank my advisor, Dr. Christopher Wyatt, for his guidance, help and commitment throughout my study here at Virginia Tech. I have been given great encouragement and strong support during the entire PhD study. I also would like to thank all my committee members for their suggestions on my dissertation.

I extend great gratitude to my colleagues in the Bioimaging Systems Lab (BSL), many others who have together created an excellent work environment here. I would specially thank Jenny (Xiaojing) Long for all the inspiring discussions and brainstorming.

I would like to particularly thank my parents and my husband, Tao, for believing in me, and for their encouragement and greatest support. 


\section{Contents}

$\begin{array}{lll}\text { Abstract } & \text { ii }\end{array}$

Dedication $\quad$ iii

Acknowledgement $\quad$ iv

List of Figures $\quad$ viii

List of Acronyms $\quad$ XV

1 Introduction $\quad 1$

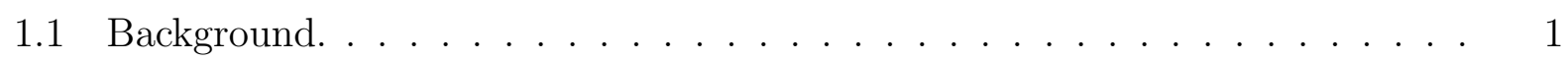

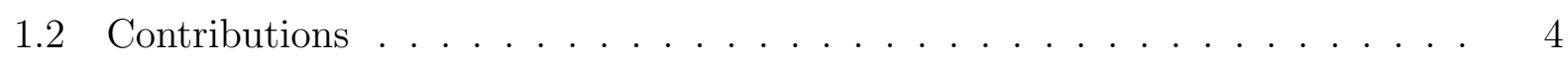

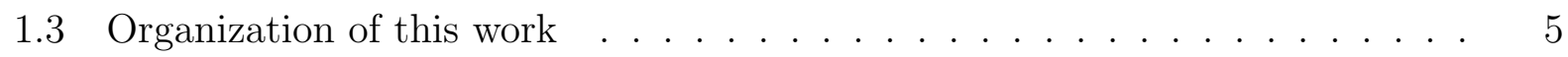

$\begin{array}{lll}2 & \text { Related work and Preliminaries } & 6\end{array}$

2.1 Deformable registration algorithms for images with topological changes . . . 7

2.2 Diffusion-based deformable registration algorithms . . . . . . . . . . . . . . 9

2.2.1 Important physical constraints . . . . . . . . . . . . . . . . . . . 9

2.2 .2 Demons algorithms . . . . . . . . . . . . . . . . . 12

2.2.3 Large Deformation Diffeomorphic Metric Mapping algorithms . . . . 15 
3.1 Impact of topological changes on deformable registration methods . . . . . . 21

3.2 A simple algorithm to suppress the impact of topological change through in-

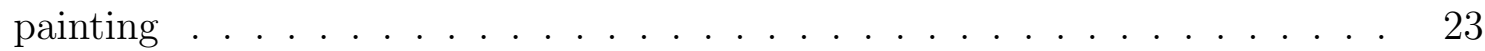

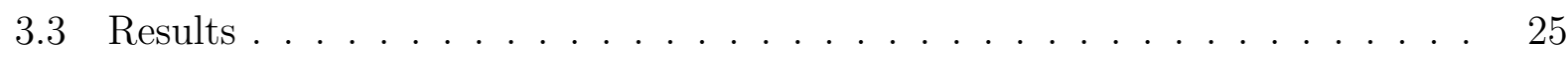

4 Registration of Images with Varying Topology using Embedded Maps 30

4.1 Preliminaries . . . . . . . . . . . . . . . . . . . 31

4.2 Registration method . . . . . . . . . . . . . . . . 33

4.2 .1 Choice of embedding . . . . . . . . . . . . . . . . 33

4.2.2 Computation of surface variation . . . . . . . . . . . . 35

4.2 .3 Diffeomorphism . . . . . . . . . . . . . . . . . . 37

4.2.4 Registration algorithm . . . . . . . . . . . . . . 38

4.3 Implementation details . . . . . . . . . . . . . . . . . . . . . . . . . . . 39

4.4 Experiments and results . . . . . . . . . . . . . . . . . . 42

4.4.1 Register template to brain MRIs with lesions. . . . . . . . . . . . 42

4.4.2 Register brain MRIs of healthy subject to that of Alzheimer's patients. 52

4.4.3 Impact of false alarm and mis-detection of lesion. . . . . . . . . . . . 54

4.4.4 Example of registering a template to a brain image with a tumor. . . 63

4.4.5 Registering images of normal topology. . . . . . . . . . . . . 67

4.4 .6 Smoothness of $\beta \ldots \ldots \ldots \ldots$. . . . . . . . . . . . . . . . . 72

4.4.7 Comparison with in-painting. . . . . . . . . . . . . . . . 72

5 Conclusion and Future Work $\quad 79$

5.1 Research summary . . . . . . . . . . . . . . . . 79

5.2 Future work . . . . . . . . . . . . . . . . . . . . 80 
B Impact of $\beta$ 


\section{List of Figures}

1.1 Examples of brain MR images with local intensity change. (a): a healthy brain. (b): a brain with MS lesion. (c): a brain with LA lesion. (d): a brain with meningioma, one type of brain tumor. . . . . . . . . . . 2

1.2 Illustration of topological changes to the level-sets of brain MRI under MS. (a) and (b): a pair of synthesized brain MR T1-weighted images with and without a simulated MS, respectively. (c) and (d): iso-contour plots of (a)

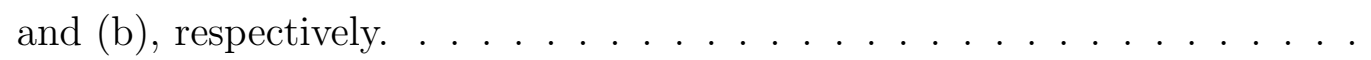

2.1 Illustration of the Diffeomorphism. (a) Original grids, $\phi=$ Id. (b) Deformed grid with a diffeomorphism. (c) Deformed grid with a non-diffeomorphism.

2.2 Illustration of the symmetry. The registration is inverse consistent when $\phi^{-1}=$

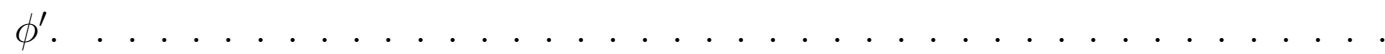

3.1 Registration of simple images with topological change. (a): moving image $I_{m}$ (b): fixed image $I_{f} ;(\mathrm{c})$ : glyph view of the deformation field generated from diffeomorphic demons; (d): zoom-in view of (c) around the region with topological change; (e): the deformation field generated from symmetric LDDMM;

(f) zoom-in view of (e) around the region with topological change. . . . . . .

3.2 Illustration of our registration method. (a): total difference Diff $t_{t}$ obtained after the coarse registration; (b): deformed $I_{1}$ into the image space of $I_{m} ;(\mathrm{c})$ : the edge map GM of (b); (d): the probability map $P$ of topological changes in $I_{m} ;(\mathrm{e})$ : binary label map of topological changes in $I_{m} ;(\mathrm{f}): I_{m}^{\prime}$ obtained after repairing; $(\mathrm{g})$ : the deformation field obtained after the fine registration; (h): the zoom-in view of $(\mathrm{g})$ around the topological change in $I_{m} . \quad \ldots$. . . 
3.3 Registration results of brain MRIs with MS, using our proposed method. (a): target image $I_{f} ;(\mathrm{b})$ : source image $I_{m}$ which contains MS and after a nonlinear deformation from $I_{f} ;(\mathrm{c})$ : probability map of topological change P; (d): binary map of detected lesion in $I_{m}$; (e): an axial slice of $I_{m} ;(\mathrm{f})$ and $(\mathrm{g})$ : the magnitude of the deformation field at the same slice as in (e), after the coarse and fine registrations, respectively. Inside the green boxes: regions with MS lesions. Regions within green boxes are enlarged at the bottom. . . . . . . .

3.4 Samples of in-painting results of lesion segmented by FreeSurfer. (a) and (b): each gives Axial, Sagittal and Coronal views of one in-painting example. In both (a) and (b), the first row shows the brain MR image with severe MS lesion. The direct in-painting repaired results are given in the second row. In addition, the third row shows the desired lesion repair results, obtained using our deformable registration algorithm presented next in Chapter 4 . . . . . .

4.1 Embedding and the length element [1]. A 2D image is embedded into a $3 \mathrm{D}$ surface, where the third dimension is the image intensity $I$. The length element $d s$ on the embedded surface is to be computed via the metric $g$. . . 32

4.2 Influence of $\beta$ on the deformation field. . . . . . . . . . . . . . . . . 34

4.3 Registration Residual for the first set of registrations. (a) Sample curves plotting the registration residual during the registration process. The solid line plots the image $L_{2}$ residual after the spatial deformation field of the current iteration is applied on the moving image, and the solid line plots the image $L_{2}$ residual when the intensity displacement is also applied. (b) Image $L_{2}$ residuals before and after registration. The blue bars shows the $L_{2}$ distance between the source and target image before deformable registration. The red bars show the $L_{2}$ residual between spatially deformed moving image and the target images. The green bars show the $L_{2}$ residual after the intensity displacement is further applied to the target image. . . . . . . . . 
4.4 Sample of registration results 1: from a template to subjects with lesion from OASIS dataset. The first row shows the template image which is affinely aligned to the target image. The second row shows the target images that containing lesions, which are marked out with blue contours. The third row shows the spatially deformed template image, and the last row gives the target image after intensity displacement is applied. The fifth and sixth rows plot the iso-contours of the moving image on top of the target image, before and after the spatial deformation is applied, respectively. The columns show the Axial, Sagittal and Coronal views, from left to right, respectively. . . . . . .

4.5 Sample of registration results 2. Subfigures are organized in the same way as in Fig. 4.4. . . . . . . . . . . . . . . . . .

4.6 Sample of registration results 3. Subfigures are organized in the same way as in Fig. 4.4. . . . . . . . . . . . . . . . . . . . .

4.7 Sample of registration results 4. Subfigures are organized in the same way as in Fig. 4.4. . . . . . . . . . . . . . . . . . . .

4.8 Samples of the resulting deformation vector fields from a template to subjects with lesion from OASIS dataset. Vector fields are shown using glyphs. Leftmost column: the template image. Second column from left: two subjects with severe lesions. Third column from left: the spatially deformed template image after registration. Right-most column: zoomed view of deformation fields on top of the target image. For the first subject (upper row), zoomed region around the first ventricle. For the second subject (lower row), zoomed region around the lateral ventricle. . . . . . . . . . . . . . .

4.9 First example of comparing our registration result with that of diffeomorphic demons. The first column from left shows the template image, the second column shows the target image. The third column gives the result of our algorithm and the right-most column gives that from diffeomorphic demons. The first and third row shows the images in Axial and Coronal views, respectively. The second and fourth row shows the glyph plot of deformation fields in Axial and Coronal views, respectively. . . . . . . . . . . . . .

4.10 Another example of comparing our registration result vs. diffeomorphic demons. Subfigures are organized in the same way as in Fig. 4.9. . . . . . . . . 
4.11 Registration residual for the second set of registration. (a): Sample curves plotting the registration residual during the registration process. The solid line plots the image $L_{2}$ residual after the spatial deformation field of current iteration is applied on the moving image, and the solid line plots the image $L_{2}$ residual when the intensity displacement is also applied. (b): Image $L_{2}$ residuals before and after registration. The blue bars shows the $L_{2}$ distance between the source and target image before deformable registration. The red bars show the $L_{2}$ residual between spatially deformed moving image and the target images. The green bars show the $L_{2}$ residual after the intensity displacement is further applied to the target image. In addition, the magenta bars in this subfigure show the $L_{2}$ residual after apply diffeomorphic demons

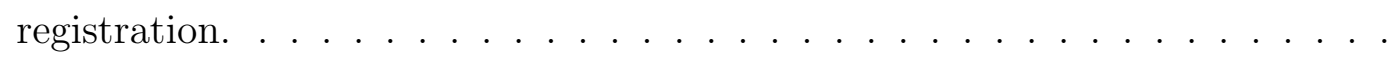

4.12 Sample of registration results 1: from a template to subjects with lesion from ADNI dataset. The first row shows the template image which is affinely aligned to the target image. The second row shows the target images that contain lesions, which are highlighted with blue contours. The third row shows the spatially deformed template image, and the last row gives the target image after intensity displacement is applied. The fifth and sixth rows plot the isocontours of the moving image on top of the target image, before and after the spatial deformation is applied, respectively. The columns show the Axial, Sagittal and Coronal views, from left to right, respectively. . . . . . . . . . .

4.13 Sample of registration results 2. Subfigures are organized in the same way as in Fig. 4.12. . . . . . . . . . . . . . . .

4.14 Sample of registration results 3. Subfigures are organized in the same way as in Fig. 4.12. . . . . . . . . . . . . . . . .

4.15 Sample of registration results 4. Subfigures are organized in the same way as in Fig. 4.12. . . . . . . . . . . . . . . . . . . .

4.16 Sample of difference images 1 . The first row shows the difference images between the moving and target images before registration. The second row gives the difference images between the deformed moving image and the target image. The third row provides the difference images between the deformed moving image and the lesion-corrected target image. . . . . . . . . . . 
4.17 Sample of difference images 2. Subfigures are organized in the same way as in Fig. 4.16. . . . . . . . . . . . . . . . . . . . . . .

4.18 Impact of imprecise segmentation of lesion. In both (a) and (b), the subfigures in the upper row, from left to right, show an axial view of the moving image, the target image, the registered moving image (after spatial deformation) using our algorithm, and the registered moving image (after spatial deformation) using diffeomorphic demons. Also, in both (a) and (b), the left most subfigure in the lower row indicate a region that we zoom in to visualize the deformation field. The second and third subfigures from the left gives the glyph view of the deformation field obtained using our method and diffeomorphic demons, respectively. . . . . . . . . . . . . . . . . . . . .

4.19 Sample of registration with imprecise segmentation. (a): lesion segmentation obtained using FreeSurfer, lesion areas are highlighted using blue contours, with part of lesions underestimated. (b): the constructed $\beta$ function. (c): result after intensity displacement applied on the target image. (d): the

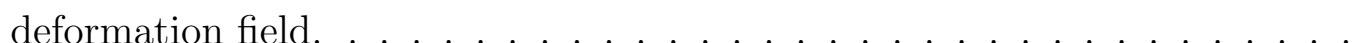

4.20 False deformation caused by the miss detection of topological change. . . . . 64

4.21 Sample registration when both moving and target image carry lesions. (a): moving image with lesion. (b): target image with lesion. (c): deformed moving image after spatial deformation. (d): the deformation field. . . . . .

4.22 Sample registration of a brain template to the MRI of a meningioma patient. First row: a brain template, the moving image. Second row: target image, MRI of a meningioma patient. Third and fourth row: checkerboard image of the target and the moving image, before and after spatial deformation, respectively. The fifth row: the intensity corrected target image after intensity displacement. The sixth row: glyph plot of the resulting deformation field. The 3 columns show the Axial, Sagittal and Coronal views of the same image. Note that the length of glyph is amplified for better visualization and is not in real pixel scale. . . . . . . . . . . . . . . . . . . . . . . 
4.23 Sample result of registering the elderly template to a young and healthy subject from OASIS. The first and second rows give the checkerboard image of the target image and the template, before and after applying spatial deformation, respectively. The third and fourth row plot the iso-contours of the template, before and after registration, respectively, on top of the target image. . . . .

4.24 Another sample of registering the elderly template to a young and healthy subject from OASIS. Subfigures are organized in the same way as in Fig. 4.23. 69

4.25 Sample result of registering the elderly template to a healthy elderly subject from OASIS. Subfigures are organized in the same way as in Fig. 4.23. . . . . 70

4.26 Sample result of registering a young and healthy subject from OASIS to another. Subfigures are organized in the same way as in Fig. 4.23. . . . . . . .

4.27 Curves plotting image $L_{2}$ residuals during the registration with $\sigma_{\text {prob }}=0$ and $\sigma_{\text {prob }}=1 \ldots \ldots \ldots \ldots \ldots \ldots \ldots \ldots \ldots \ldots \ldots \ldots \ldots \ldots \ldots \ldots$

4.28 Example showing the impact of smoothing kernel $K_{\text {prob }}$. (a): The first row shows the checkerboard image of the template and target image before registration. The second and third row show the registered template after applying spatial deformation, using $\sigma_{\text {prob }}=0$ and $\sigma_{\text {prob }}=1$, respectively. (b): the first row shows a zoom-in view of the region with lesion in the target image, where the boundary of FreeSurfer lesion segmentation was marked by blue contours. The second and third rows show the lesion-repaired target images using $\sigma_{\text {prob }}=0$ and $\sigma_{\text {prob }}=1$, respectively. . . . . . . . . . . . . . . 74

4.29 Sample registration result using our algorithm. First row: moving image. Second: target image. Third row: spatial deformed moving image. . . . . . .

4.30 On the same registration experiment as in Fig. 4.29, registration result when in-painting is used. First row: moving image after in-painting. Second: target image after in-painting. Third row: spatial deformed moving image, i.e., after apply the deformation field obtained using diffeomorphic demons on the inpainted image. . . . . . . . . . . . . . . . . . . . 
4.31 Impact of in-painting. (a) glyph view of the deformation field obtained using diffeomorphic demons after in-painting (left) and our algorithm (right). (b) upper row: result when the deformation field obtained using in-painting is applied on the original moving image; lower row: result when the deformation field obtained using our algorithm is applied on the original moving image. .

5.1 Classification on dataset using whole-brain distances. Quick shift classification result in the embedded space [2]. 


\section{List of Acronyms}

$\begin{array}{ll}\text { MRI } & \text { magnetic resonance imaging } \\ \text { DTI } & \text { diffusion tensor imaging } \\ \text { MS } & \text { multiple sclerosis } \\ \text { LA } & \text { leukoaraiosis } \\ \text { DBM } & \text { deformation based morphomertry } \\ \text { WM } & \text { white matter } \\ \text { GM } & \text { gray matter } \\ \text { CSF } & \text { cerebrospinal fluid } \\ \text { LDDMM } & \text { large deformation diffeomorphic metric mapping algorithms } \\ \text { PDE } & \text { parital differential equation } \\ \text { HDW } & \text { high dimensional warping }\end{array}$




\section{Chapter 1}

\section{Introduction}

\subsection{Background.}

Deformable registration algorithms use dense vector fields to capture detailed structural differences between anatomical brain images from magnetic resonance imaging (MRI). The resulting deformation fields are extremely informative in subsequent clinical studies of brain shapes, such as deformation based morphometry (DBM), both in terms of cross-subject variation and disease progression. Therefore, the design of robust deformable registration algorithms is regarded as an important research topic and has attracted significant research interest $[3,4,5,6,7,8]$.

However, many brain diseases or even natural aging will result in intensity changes in certain regions of anatomical brain images. For example, white matter multiple sclerosis (MS) lesions appear to be dark spots in T1-weighted MR images, which are referred to as hypointensity lesions. Leukoaraiosis (LA) lesions appear to be brighter than normal white matter in T2-weighted images, and are referred to as hyperintensity white matter lesions. Tumors can have different appearances in MR images; depending upon which type they belong to. Nevertheless, they usually will appear to be much brighter than normal brain tissue when imaged with contrast agents. In Fig. 1.1, we provide some examples of MR images with these diseases to illustrate how they cause some local intensity changes to certain regions of the brain image.

Ideally, medical images with the same field of view of healthy individuals contain the same anatomical objects, whose level-sets should have the same topology, up to minor differences 


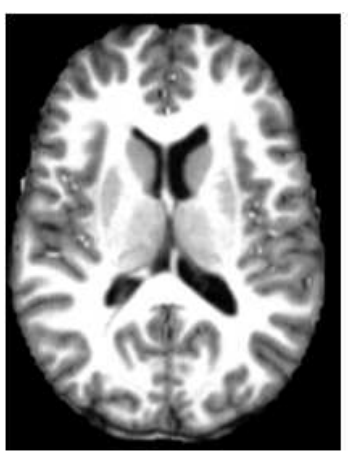

(a)

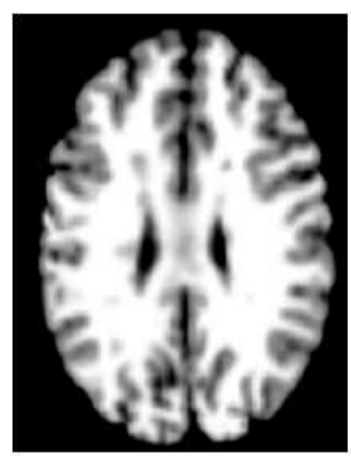

(b)

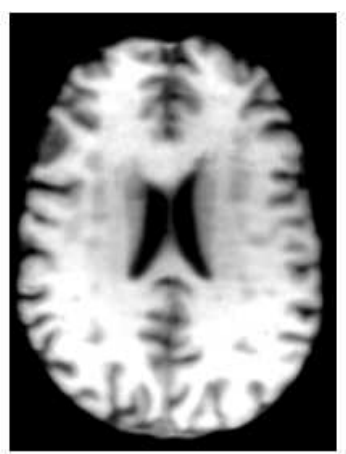

(c)

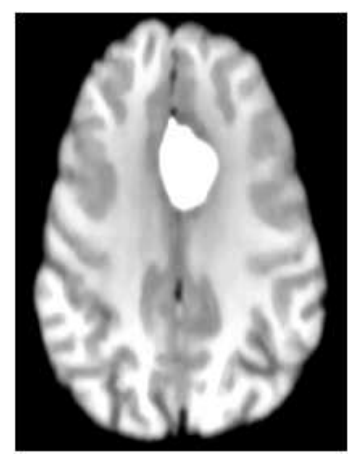

(d)

Figure 1.1: Examples of brain MR images with local intensity change. (a): a healthy brain. (b): a brain with MS lesion. (c): a brain with LA lesion. (d): a brain with meningioma, one type of brain tumor.

caused by noise. However, in the presence of intensity change caused by brain diseases, the topology of the image level-sets will be changed (which is what we will refer to as topological changes throughout this work.). Fig. 1.2 gives an example of such topological change, where (a) and (b) show a pair of synthesized brain MR T1-weighted images with and without a simulated MS, respectively, while (c) and (d) give the iso-contours of the two brain images in (a) and (b), respectively. Note that topological changes to their respective level-sets are clearly visible, as pointed out by the arrows in the figure.

Such topological changes can cause false deformation (refer to Sec. 3.1) in the resulting dense vector fields from existing deformable registration algorithms [9]. For the subsequent analysis, e.g., DBM, that relies on the dense vector fields, false deformation will be wrongly associated with local growth or shrinkage [10]. In existing literature, the issue of suppressing the impact of topological changes on deformable registration has been approached in three different ways:

- Pre-processing: the lesion-affected regions are removed and the remaining regions are filled via in-painting. Deformable registration is then performed on the repaired images.

- Blend-into-registration: the registration algorithm can be particularly designed to behave differently when lesion is encountered. Ideally, identified lesions shall not cause any spatial deformation.

- Post-processing: regular deformable registration algorithms are directly applied on 


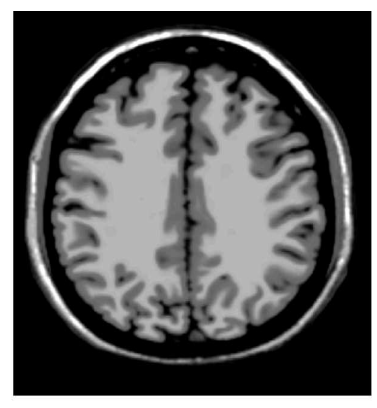

(a)

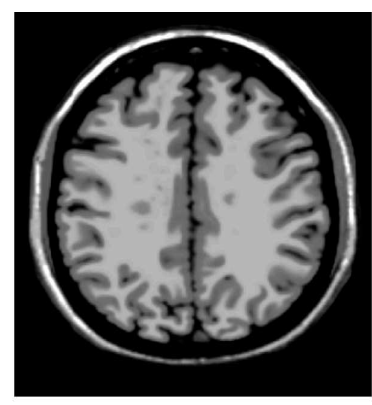

(b)

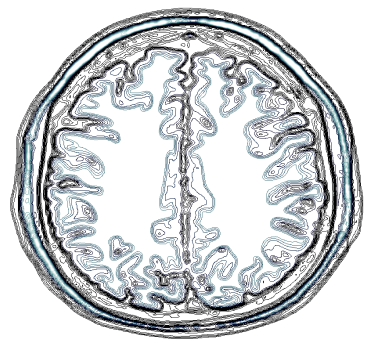

(c)

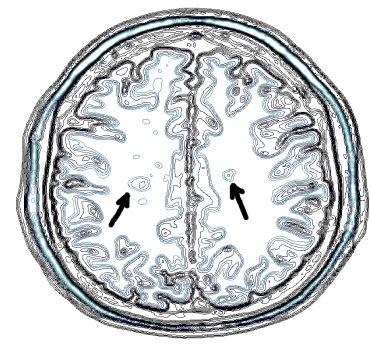

(d)

Figure 1.2: Illustration of topological changes to the level-sets of brain MRI under MS. (a) and (b): a pair of synthesized brain MR T1-weighted images with and without a simulated MS, respectively. (c) and (d): iso-contour plots of (a) and (b), respectively.

images with topological changes. False deformation is to be removed afterwards.

Of the three ways of handling topological changes, post-processing is regarded as the most difficult for the following two reasons. First, deformation fields that are useful in clinical analysis need to satisfy certain constraints, e.g., diffeomorphic (refer to Sec. 2.2.1). After modifying part of the deformation field in the false-deformation-removal step following deformable registration, it is difficult to guarantee that such constraints are still satisfied. Second, constraints such as diffeomorphism need to be ensured by smoothness constraints. As a result, the impact of lesion on the deformation field is not focal, even when the lesion appears to be focal, and can spread over a large region, depending on the image contrast. Hence, identifying the boundary of a false deformation is very difficult, if not impossible. Due to these concerns, the majority of methods handle false deformation for deformable registration using the first and second categories, i.e., preprocessing and blend-into-registration. In this work, we take the second approach and design a registration algorithm that handles topological changes naturally during the registration process. Compared with the pre-processing methods based on in-painting, our algorithm has the following advantages:

- First, in-painting cannot repair lesions which cover regions of non-uniform intensity profiles, such as part of both white matter (WM) and gray matter (GM). Our algorithm, on the other hand, can copy the texture from the registered moving image. As a result, our algorithm can also be used to register images with tumors and after resection, which cannot be handled by in-painting based methods. 
- Second, in-painting is an additional pre-processing step, requiring a sophisticated algorithm with high computational cost. On the other hand, our algorithm is an integrated process which corrects the topological change (as a by-product) during registration.

\subsection{Contributions}

Considering the state-of-the-art of deformable registration algorithms, our contributions in this work are summarized as follows.

First, using two state-of-the-art deformable registration algorithms, namely, diffeomorphic demons and large deformation diffeomorphic metric mapping algorithms (LDDMM), we specifically show the impact of topological changes on their performance and thus demonstrate the necessity of handling topological changes. In particular, we use several simple cases to demonstrate the false deformation issue associated with the two algorithms and design a simple in-painting-based tool to show the possibility of removing false deformation in the targeted brain regions. However, it is demonstrated that this simple method is not robust enough to handle real-world data and thus not practical for clinical applications.

Second, we design a new deformable registration algorithm based on embedded maps that is capable of accurately registering images with considerable structural differences while suppressing the impact of topological changes on the deformable registration process. In our proposed algorithm, 3D images are embedded as 4D surfaces in a Riemannian space. The registration is therefore conducted as a surface evolution, which is modeled by a diffusion process. Our algorithm differs from existing methods in the sense that it takes a priori estimation of areas with topological change as an additional input and generates dense deformation vector fields which are free of false deformation. In particular, the output of our algorithm consists of a diffeomorphic deformation field and an intensity displacement which corrects intensity difference caused by topological changes. By conducting extensive experiments, we demonstrate that our proposed algorithm generates accurate registration results for images with considerable topological changes caused by different pathologies. More importantly, the resulting deformation field is not impacted by topological changes, i.e., there is no false deformation. 


\subsection{Organization of this work}

The remainder of this work is organized as follows. In Chapter 2, we give a review on existing deformable registration algorithms. We first review the currently available registration algorithms that focus on handling topological changes. Then, we review some of the diffusion-based deformable registration algorithms that confine the output of the registration algorithm to be a diffeomorphic dense vector field. These works use similar physical models to our algorithm and thus are used for comparison purposes. In Chapter 3, we use experiments to deonstrate the the false deformation that caused by topological changes using existing deformable registration algorithms and investigate the cause of the false deformation. We designed a simple in-painting-like tool to show the possibility of removing false deformation for the targeted regions with topological changes. In Chapter 4, we present our new deformable registration algorithm based on embedded maps. The proposed algorithm is extensively tested on multiple sets of experiments. The registration results are compared to other algorithms where topological changes are not specifically handled. Chapter 5 concludes the dissertation and propose some future work. 


\section{Chapter 2}

\section{Related work and Preliminaries}

Registration refers to the process of determining a spatial transform that maps points in one image - source (moving) image - to their homologous points of the same object in another image - target (fixed) image [11]. Among the vast number of registration methods, such as rigid registration and affine registration, deformable registration allows an image to be warped with high degree of freedom. Deformable registration has been found to pick up detailed structural differences among imaged subjects, and thus has triggered great research interest $[12,13]$. Deformable registration can be modeled in many different ways, which lead to distinct optimization processes and result in deformation fields of different degrees of elasticity. Over the past four decades or so, a huge number of deformable registration methods have been proposed and investigated, many of which have further evolved into different versions. In the past, several algorithms have been proposed to perform registration via surface labeling or marker matching [14, 15, 16], and demonstrated encouraging registration performance. Especially, FreeSurfer [17] is a well designed surface-registrationbased pipeline, which is composed of bias-correction, skull stripping, non-linear smoothing, gray/white matter segmentation, surface unfolding/flattening and morphing. A similar approach is High-Dimensional Image Warping (HDW) [18], where the deformation field is parameterized as piecewise affine transformations within a finite element mesh. Incorporating smoothing constraints, the registration is modeled as a process of finding the maximum a posterior (MAP) estimate of the parameters.

The transformations in spline-based deformable registration methods are defined by a linear combination of a set of basis functions, including thin-plate spline (TPS), elastic body splines (EBS), or B-splines. Among them, B-spline is the most commonly used one [19, 20, 21]. For 
B-spline-based methods, the degree of flexibility is confined by the spacing of the grid of control points. Compared with algorithms using non-parametric transformations, which rely on a function defined on certain physical properties of images to guide the registration process, B-spline-based methods have only limited degrees of freedom, and certain constraints, e.g., smoothness, can be easily imposed. However, as a trade-off, the elasticity of the deformation is also limited and thus it may not be able to represent subtle structural changes that are finer than the B-spline basis grid.

Aware of many different deformable registration algorithms, in this chapter, however, we only focus our review on the methods that are closly related to our work, i.e., either using a similar diffusion model, or trying to handle a similar problem. For a complete review on deformable registration algorithms, we refer interested readers to survey papers $[3,4,5,6,7,8]$.

\subsection{Deformable registration algorithms for images with topological changes}

As introduced in Chapter 1 , it is very common to see medical images with topological changes in clinical studies. The most frequently seen brain diseases that relate to topological changes are multiple sclerosis (MS), leukoaraiosis, Alzheimer's, meningioma etc. All these diseases are currently attracting intense research efforts in clinical studies. Algorithms such as deformation-based morphometry (DBM) that rely on the dense vector fields are widely used in many large scale studies, which, however, is shown to be affected by lesion induced false deformation [10]. Specifically, when the topology of the moving and the fixed images do not agree, the diffusion process will attempt to shrink or expand a region with topological change, depending on the intensity contrast, in order to minimize the intensity residual as modeled by the objective function. However, topological changes present in brain MRI can be caused by different pathologies. For example, the growth of tumors pushes the surrounding tissue aside. MS and Leukoaraiosis usually cause density changes of white matter (and density change in gray matter as well in the case of stroke), which in fact, is independent of brain structural change. Consequently, during deformable registration, the spatial deformation that is generated to shrink or expand the topological changes is incorrect. As a result, we refer to the topological-change-induced deformation as false deformation. In addition, despite the shrinked area, regions with topological change will not be completely eliminated by spatially deforming the region. Instead, the shirnking region will persist with 
its mass condensed during the entire registration process. Thus, sources that are driving false deformation will persist throughout the diffusion process. As a result, the energy caused by false deformation is correlated to the length of the diffusion. The longer the diffusion process is, the stronger the false deformation is.

In the past, in-painting was a commonly used method in DBM to remove false deformation [10, 22]. However, as we discussed in Chapter 1, these works require a reasonably accurate segmentation of lesions and a possibly sophisticated in-painting algorithm.

A notable algorithm that models image intensity evolution is metamorphosis, proposed in [23]. A metamorphosis is a Riemannian metric defined on the space of images to account for both geometric deformation and intensity changes. It resembles an image morphing process and is a pair of curves corresponding to a spatial deformation and a template evolution, respectively. Using the concept of metamorphosis, topological changes can be accommodated by template evolution, i.e., intensity displacement, which leads to a smooth diffusion even in the presence of severe topological changes. Therefore, it has been used in facial morphing, medical image averaging [24], and brain atlas estimation [25]. However, in metamorphosis, input images are set to be the base points of the deformation. Topological changes will impact both spatial deformation and intensity displacement in this registration process. As a result, false deformation is still expected to be present in the deformation, i.e., metamorphosis is not specially designed to solve the false deformation problem.

Alternatively, some registration algorithms use pre-computed pathology models to handle topological changes [26]. However, the pre-computed model has limited application to specific pathology types, e.g., a certain type of tumor. Note that brain resections or the presence of neurosurgical instruments can also be regarded as topological changes in general. Several works are developed to register brain images with resections by matching subvolumes [27, 28], landmarks [29] or segmentation surfaces [30]. In addition, the ExpectationMaximization (EM) framework has been used for a joint estimation of resection region and registration $[31,32]$. These works rely on local features, and in most cases, their registration results have limited dimensionality rather than a dense deformation field, due to the computational complexity of EM. The work by Risholm et al. [29] uses anisotropic diffusion instead of Gaussian smoothing in a registration framework that generates a deformation field that is free of the impact from resections. In their work, the anisotropic diffusion is regulated by diffusion sinks, where it is permitted into the area (resection area) but not out. In general, compared to resection, other topological changes can occur in many regions of a brain image. For instance, brain images of MS patients can contain many small regions with 
hypointensity. Each region then needs to be modeled as a separate diffusion sink, which will induce heavy computational load to the registration process. The registration algorithm by Cuzol et al. [33] models the topological change using a brightness variation model. However, with the additional luminance change function, the registration needs to be solved with a generalized conjugated gradient process, which is not as stable as a strict gradient descent process, and does not guarantee convergence to a true local minimum.

We need to point out that, there are other commonly seen intensity non-uniformities on medical images, such as bias and noise. Bias is typically of low frequency and spread over the entire image. Noise, on the other hand, is high frequency and possibly non-stationary. Improved performance in many medical image processing tasks, including registration, is linked to improved bias and noise correction. As a result, bias and noise has been studied in previous work $[34,35]$ and can be included in the preprocessing of clinical data. In our work, unless segmented in the label map, bias and noise are not regarded as topological changes. In fact, this segmentation will be very hard, especially for bias. However, as will be introduced in Chapter 4, during the registration process, if the moving and target images are well aligned and the diffusion is not terminated, the intensity difference caused by bias and noise will be corrected to a certain extent.

\subsection{Diffusion-based deformable registration algorithms}

In this section, we introduce some important constraints in deformable registration, i.e., diffeomorphism and symmetry. Their physical meaning and mathematical forms are explained in detail. Then we study two families of state-of-the-art deformable registration algorithms, i.e., diffeomorphic demons and LDDMM.

\subsubsection{Important physical constraints}

In deformable registration, only a subgroup of all deformation fields is regarded as realistic and useful. In other words, certain constraints are crucial and need to be satisfied in the deformation fields obtained from deformable registration algorithms. The first one is Diffeomorphism. Mathematically, a diffeomorphism has the following definition [36, 37, 38]: 
Diffeomorphism: given two manifolds $M$ and $N$, a bijective map $f$ from $M$ to $N$ is called a diffeomorphism if both

$$
f: M \rightarrow N
$$

and its inverse

$$
f^{-1}: N \rightarrow M
$$

are differentiable (if these functions are $r$ times continuously differentiable, $f$ is called a $C^{r}$-diffeomorphism).

Two manifolds $M$ and $N$ are diffeomorphic if there is a smooth bijective map $f$ from $M$ to $N$ with a smooth inverse. They are $C^{r}$ diffeomorphic if there is an $r$ times continuously differentiable bijective map between them whose inverse is also $r$ times continuously differentiable.

Diffeomorphism is an important physical constraint in image registration. From an anatomical perspective, a diffeomorphism ensures that there is no tearing or folding of the physical space. For an image, it prevents the image from folding the grid over itself and thus destroying neighborhood structure. Also, a diffeomorphism guarantees the smoothness of the deformation field by eliminating spikes, which leads to a stable PDE evolution in diffusionbased methods. Fig. 2.1 gives an example to demonstrate the warping results of an image grid by a diffeomorphic and a non-diffeomorphic vector field. The original image grid in 2-D is given in Fig. 2.1(a). After deforming with a vector field that is diffeomorphic, although the image grid has been distorted, as shown in Fig. 2.1(b), all the pixels stay in the same order in both the horizontal and the vertical directions as in the original grid. On the other hand, as shown in Fig. 2.1(c), when the image grid is deformed with a deformation field that is not diffeomorphic, then the order of the pixels in the horizontal axis has been broken, and thus the grid folds over itself. Furthermore, for the folded area, the spatial grid and the image intensity form a one-to-many mapping, which violates the fundamental physical formation of an image.

It is well known that diffeomorphisms do not form a vector space, but only a Lie group [13]. A Lie group is a group which is also a differentiable manifold, with the property that the group operations are compatible with the smooth structure. Lie groups are smooth manifolds and are studied using differential calculus. In discrete applications, smoothing is always needed to ensure the diffeomorphism, such examples will be given later when we examine two existing algorithms in Sec. 2.2.2 and Sec. 2.2.3. 


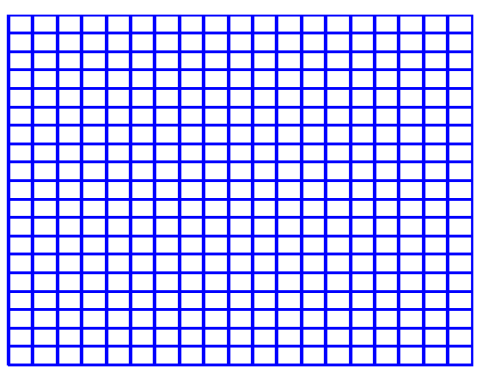

(a)

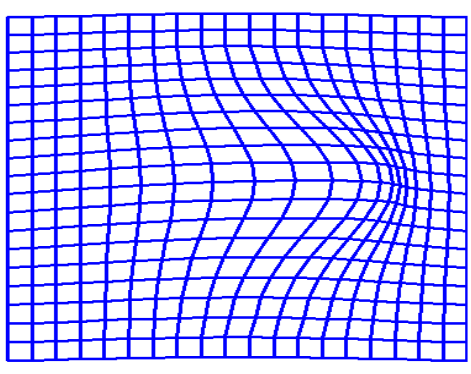

(b)

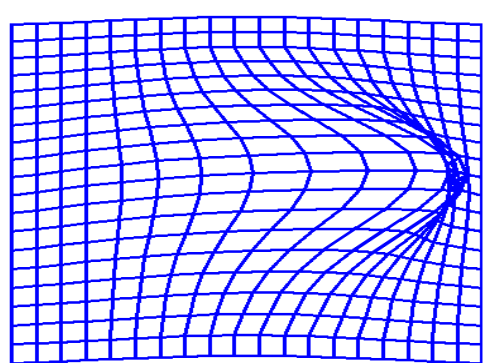

(c)

Figure 2.1: Illustration of the Diffeomorphism. (a) Original grids, $\phi=$ Id. (b) Deformed grid with a diffeomorphism. (c) Deformed grid with a non-diffeomorphism.

The second constraint is called symmetry. In deformable registration, symmetry refers to the notion that the resulting deformation remains the same when the moving and the fixed images are swapped. In terms of practical implementation, symmetry can refer to symmetric gradient or inverse consistency. Symmetric gradient is imposed on each individual updating, and does not guarantee symmetry on the final deformation field. Inverse consistency, on the other hand, ensures strictly the same output regardless of the order of the moving and the fixed image. Inverse consistency is illustrated in Fig. 2.2.

To understand Fig. 2.2, it is important to be aware of the existence of registration residual. During the registration process, ideally, if the moving and the fixed images, denoted by $I_{m}$ and $I_{f}$ respectively, only differ in terms of structural changes, the resulting spatial deformation $\phi$ is capable of perfectly aligning the two images, i.e., $\left\|I_{m}-I_{f} \circ \phi\right\|_{L^{2}}=0$. However, the intensity profile of the moving and target images are typically not identical. Even in cases where the two input images have the same intensity profile, during the registration process certain physical constraints, such as smoothness or diffeomorphism, are usually imposed on the underlying deformation fields. As a result, the spatial deformation may not be able to perfectly align them, leading to certain residuals, measured as the intensity distance between the deformed moving image and the target image. Another source of such residual is the numerical errors from the interpolation scheme used in sub-pixel level computation. Formally, image residual refers to the intensity difference between the fixed and the registered moving images: $r_{0}=\left\|I_{f}-I_{m} \circ \phi\right\|_{L^{2}}$, and $r_{1}=\left\|I_{m}-I_{f} \circ \phi^{-1}\right\|_{L^{2}}$ in Fig. 2.2.

Then we get back to the concept of the inverse consistency constraint: the order of source and target images can be flipped and still result in the same deformation field, i.e., $\phi^{\prime}=\phi^{-1}$; 


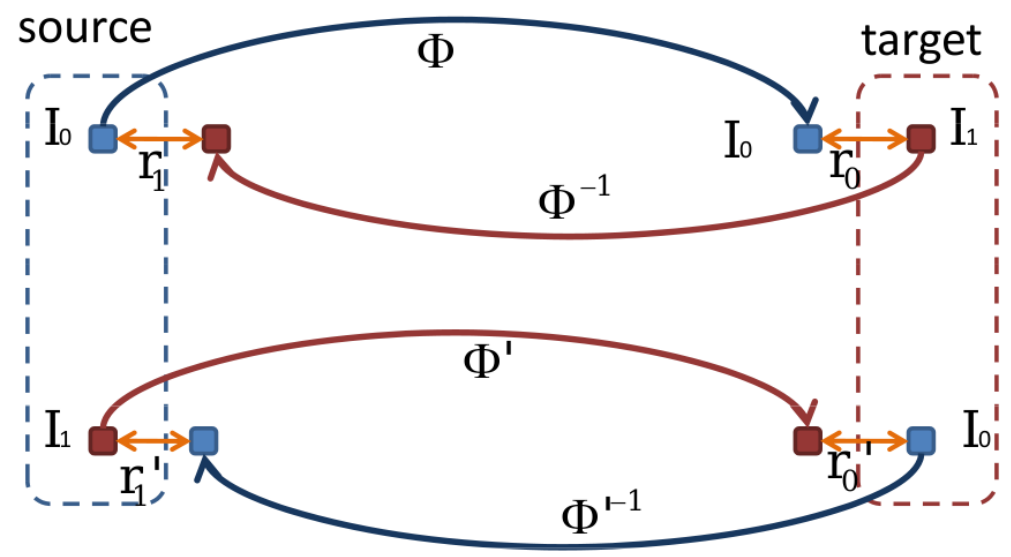

Figure 2.2: Illustration of the symmetry. The registration is inverse consistent when $\phi^{-1}=\phi^{\prime}$.

$\phi^{\prime-1}=\phi ; r_{1}=r_{0}^{\prime}$ and $r_{0}=r_{1}^{\prime}$.

Inverse consistency can be ensured by modifying the registration objective as

$$
E_{\text {inverse consistent }}=\frac{1}{2}\left(E\left(I_{f}, I_{m} \circ \phi\right)+E\left(I_{m}, I_{f} \circ \phi^{-1}\right)\right),
$$

which is important when the deformation field is used as a metric between the two images.

Despite the power of regularizing deformation in image registration, the diffeomorphism and symmetry constraints cause or enhance errors when registering images with topological changes, since there will be no diffeomorphism which can align them. First, as we discussed, smoothing of the deformation field is always required to ensure a diffeomorphism. Therefore, the impact of topological changes on the deformation field will not be confined to the region of topological change. Rather, it will spread its impact over an enlarged neighborhood. Second, when the symmetry constraint is used in registration, topological changes will cause false deformation, regardless of whether they originate from the moving or the fixed images.

\subsubsection{Demons algorithms}

Thirion's demons algorithm [39] models the image-to-image matching as a diffusion process, which is analogous to Maxwell's demons, based on local image characteristics. The resulting force for evolving the deformation has the same form as optical flow equations, and can be 
solved using gradient descent.

Given a moving image $I_{m}$ and a fixed image $I_{f}$, the demons energy is defined as

$$
E(c, s)=\frac{1}{\sigma_{i}^{2}} \operatorname{Sim}\left(I_{f}, I_{m} \circ c\right)+\frac{1}{\sigma_{x}^{2}} \operatorname{dist}(s, c)^{2}+\frac{1}{\sigma_{T}^{2}} \operatorname{Reg}(s),
$$

where $s$ represents the function of the transformation, and $c$ is a vector field on the image grid that realizes the transformation $s$. The first term $\operatorname{Sim}\left(I_{f}, I_{m} \circ c\right)$ models the similarity between the deformed moving and the fixed images, and is sometimes referred to as the faithfulness. $\operatorname{Reg}(\mathrm{s})$ is the regularization constraint that can be imposed on the transformation, which is usually used to ensure the smoothness of $s$. dist(s,c) is the term that guarantees the quality of the realization $c$. The scalars $\sigma_{i}^{2}, \sigma_{x}^{2}$ and $\sigma_{T}^{2}$ balance the relative weights of the three terms.

For medical images, the $L_{2}$ distance is the most common choice for $\operatorname{Sim}\left(I_{f}, I_{m} \circ c\right)$ between images of the same modality. Other metrics, for example, mutual information [40] can be used to register images of different modalities. Limited by the Cartesian grid of images, the actual transformation $s$ can be only computed with the fixed resolution of vector field $c$, where the sub-pixel level forces are obtained by interpolation. Moreover, under a gradient descent framework, $s$ is updated incrementally by an update field $u$ at each iteration. Thus in practice the demons optimization of the energy functional becomes the following updating rule:

$$
u=\underset{u}{\operatorname{argmin}} E_{s}=\left\|I_{f}-I_{m} \circ(s+u)\right\|^{2}+\frac{\sigma_{i}^{2}}{\sigma_{x}^{2}}\|u\|^{2} .
$$

Based on (2.3), the demons algorithm pipeline can be written as follows:

\section{Algorithm: Demons registration:}

- Initialize the spatial transformation $s$ in the form of a vector field, (usually $I_{f}$ and $I_{m}$ are already affine registered, and $s$ is initialized as the identity.)

- Iteration till convergence

1. Based on current $s$, compute update field using

$$
u=-\frac{I_{f}-I_{m} \circ s}{\|J\|^{2}+\frac{\sigma_{i}^{2}}{\sigma_{x}^{2}}} J \quad(J \text { is a function of the image gradients })
$$

2. If choose fluid-like regularization, $u \leftarrow K_{1} * u$, where $K_{1}$ is a Gaussian smoothing convolution kernel.

3. $s \leftarrow s+u$ 
4. If choose diffusion-like regularization, $s \leftarrow K_{2} * s$, where $K_{2}$ is a Gaussian smoothing convolution kernel.

In (2.4), if $\sigma_{i}^{2}$ is set to the estimated value of image noise, controlling $\sigma_{x}^{2}$ can regularize the maximum update step. $J$ is the image gradient that indicates the direction of optical flow. In the original Thirion's rule, it is only related to the gradient of the fixed image:

$$
J=-\nabla I_{f}
$$

Another form of $J$ is defined in the Gauss-Newton method, where the gradient of the warped moving image is used

$$
J=-\nabla\left(I_{m} \circ s\right) .
$$

The concept of demons registration provides a mathematical framework for non-parametric registrations, and has quickly become popular. Over the past decade, researchers have modified the demons algorithm by imposing various constraints to render it anatomically more realistic and mathematically more stable [13,41]. Such constraints include symmetry and diffeomorphism.

As discussed in Sec. 2.2.1, there is no straightforward method to ensure diffeomorphism in the normal registration formulation, since diffeomorphisms do not form a vector space, but only a Lie group [13]. As an alternative, optimization can be performed on a Lie group, which is the space of diffeomorphisms [42, 43]. Therefore, registration results are diffeomorphic when the Lie algebra is applied during the optimization, which avoids the typical routine of adding constraints into the objective. Specifically, in the diffeomorphic demons algorithm [13], an intrinsic updating step using the classic Newton-Raphson method is applied, given by,

$$
s \leftarrow s \circ \exp (u)
$$

instead of using the updating rule in (2.4). Here, $\circ$ is composition operation, and exp is the vector field exponential. The vector field exponential can be efficiently computed for diffeomorphisms through compositions [44]:

\section{Algorithm: Fast computation of Vector Field Exponentials:}

- choose $N$ such that $2^{-N} u$ is close enough to 0 , e.g., $\max \left\|2^{-N} u\right\| \leq 0.5$;

- Perform an explicit first order integration: $v(p) \leftarrow 2^{-N} u(p)$ for all pixels;

- Do $N$ recursive squaring of $v: v \leftarrow v \circ v$. 
In order to make the inverse transform directly available, which is necessary for subsequent computations and analysis in many cases, the log-domain demons algorithm [41] has been recently proposed. This algorithm adopts the Baker-Campbell-Hausdorff (BCH) formula [45] to update the transformations as the log of composed exponentials:

$$
v \leftarrow \log (\exp (v) \circ \exp (u))
$$

Using this updating rule, both the forward and the inverse transformation are immediately accessible:

$$
s=\exp (v) \quad \text { and } \quad s^{-1}=\exp (-v)
$$

To further render the log-domain demons algorithm symmetric, the demons energy function has been modified by symmetrizing the positions of the moving and the fixed images:

$$
S=\underset{s}{\operatorname{argmin}}\left(E\left(I_{f}, I_{m}, s\right)+E\left(I_{f}, I_{m}, s^{-1}\right)\right)
$$

where $E$ stands for the typical demons energy given in (2.3). To solve this optimization, a forward and a backward update, $u^{\text {forw }}$ and $u^{\text {back }}$, are computed independently, and the resulting updating rule in the symmetric log-domain demons algorithm [41] can be written as an average of the forward and backward updates:

$$
v \leftarrow \frac{1}{2} K_{2} *\left(\log \left(\exp (v) \circ \exp \left(K_{1} * u^{\text {forw }}\right)\right)-\log \left(\exp (-v) \circ \exp \left(K_{1} * u^{\text {back }}\right)\right)\right),
$$

where $K_{1}$ and $K_{2}$ are the fluid-like and diffusion-like smoothing convolution kernels, respectively, and are the same as those used in the original demons algorithm.

\subsubsection{Large Deformation Diffeomorphic Metric Mapping algorithms}

A diffeomorphism is typically ensured by smoothing the deformation vector field. However, over-smoothing can destroy the correspondence between image details and increase registration error. To theoretically regulate the amount of smoothing on the deformation field, the works by Dupuis [46] and Trouvé [47] provide a theory on the minimal amount of smoothness required to ensure the field to be continuous and at least one time differentiable, i.e., $C^{1}$-diffeomorphic. Using this theory, the Large Deformation Diffeomorphic Metric Mapping (LDDMM) method has been proposed [48], which is capable of finding the geodesic path in the space of diffeomorphisms, between images that correspond to each other via large deformation fields. 
In the work by Beg et al. [12], a complete LDDMM algorithm and its implementation has been provided. The LDDMM algorithm solves for a geodesic deformation path $\phi_{t}$ connecting the moving image $I_{m}$ and the fixed image $I_{f}$, where it starts from $I_{m}$ at time $t=0$ and arrives at $I_{f}$ when $t=1$. This path can be computed as $\phi_{1}=\phi_{0}+\int_{0}^{1} v_{t} d t$ where $v_{t}$ is the velocity vector field of $\phi_{t}$. Using this formulation, the cost function of LDDMM has the form:

$$
\hat{v}=\arg \min _{v_{t}\left(\phi_{t}\right)=\dot{\phi}_{t}} E(v) \doteq \int_{0}^{1}\left\|v_{t}\right\|_{V}^{2} d t+\frac{1}{\sigma^{2}}\left\|I_{f} \circ \phi_{1,0}^{v}-I_{m}\right\|_{L^{2}}^{2}
$$

In (2.12), the first term models the cost of the length of the deformation, in the form of an appropriate Sobolev space $V$ which ensures enough smoothness of the velocity field. This Sobolev norm can be converted into an $L_{2}$ norm using a differential operator $L=$ $(-\alpha \Delta+\gamma)^{\alpha} I_{n \times n}(\alpha>1.5$ in $3-\mathrm{D}$ space $)$ :

$$
\|f\|_{V}=\|L f\|_{L_{2}}
$$

The second term in (2.12) reflects the data faithfulness with the deformation field $\phi$, measured in the $L_{2}$ space.

The variation of (2.12) with respect to an arbitrary perturbation $h$ on the velocity deformation field is chosen to be computed in Sobolev space $V$, using Fréchet derivative for stability, giving the Euler-Lagrange equation:

$$
\partial_{h} E(\hat{v})=\int_{0}^{1}\left\langle 2 \hat{v}_{t}-K\left(\frac{2}{\sigma^{2}}\left|D \phi_{t, 1}^{\hat{v}}\right| \nabla J_{t}^{0}\left(J_{t}^{0}-J_{t}^{1}\right)\right), h_{t}\right\rangle_{V} d t=0
$$

where $K$ is the self-adjoint operator: $K\left(L^{\dagger} L\right)=\mathrm{Id}$, and $|D \phi|$ is the Jacobian determinant of deformation field $\phi$ :

$$
|D \phi|=\left|\begin{array}{cccc}
\frac{\partial \phi_{1}}{\partial x_{1}} & \frac{\partial \phi_{1}}{\partial x_{2}} & \ldots & \frac{\partial \phi_{1}}{\partial x_{n}} \\
\frac{\partial \phi_{2}}{\partial x_{1}} & \frac{\partial \phi_{2}}{\partial x_{2}} & \ldots & \frac{\partial \phi_{2}}{\partial x_{n}} \\
& \ldots & \\
\frac{\partial \phi_{m}}{\partial x_{1}} & \frac{\partial \phi_{m}}{\partial x_{2}} & \ldots & \frac{\partial \phi_{m}}{\partial x_{n}}
\end{array}\right| .
$$

Since $h$ is a random perturbation, the unique solution to (2.15) is given by

$$
\left(\nabla_{\hat{v}} E_{t}\right)_{V}=2 \hat{v}_{t}-K\left(\frac{2}{\sigma^{2}}\left|D \phi_{t, 1}^{\hat{v}}\right| \nabla J_{t}^{0}\left(J_{t}^{0}-J_{t}^{1}\right)\right)
$$

The LDDMM algorithm can be summarized as follows:

Algorithm LDDMM: Initialize at iteration $k=0$ : $($ when $N=20, \delta t=1 / N=1 / 20)$ 
- $v_{t_{j}}^{k}=0$;

- $\nabla_{v_{t_{j}}^{k}} E_{t_{j}}=0$;

- $\phi_{t_{j}, 0}=I d$

- $\phi_{t_{j}, T}=I d$

- $K=0$.

Iteration $k+1$.

1. Calculate the new estimate of velocity at each time interval:

$$
v_{t_{j}}^{k+1}=v_{t_{j}}^{k}-\epsilon \nabla_{v_{t_{j}}^{k}} E_{t_{j}}
$$

2. Reparametrize the velocity field to be a constant speed after every 10 iterations

$$
\begin{array}{r}
s_{t}=\frac{T}{\int_{0}^{T}\left\|v_{t}\right\|_{V} d t} \int_{0}^{t}\left\|v_{t}\right\|_{V} d t \\
\dot{h_{t}}=\frac{1}{\dot{s}_{h_{t}}}, \tilde{v}_{t}=\dot{h}_{t} v_{h_{t}}
\end{array}
$$

* an additional step: Compute all $K$.

3. Calculate for $j=N-1$ to $j=0$ the mapping $\phi_{t_{j+1}, T}^{k+1}(y)$ using:

$$
\phi^{v_{t_{j}, T}^{k+1}}(y)=\phi_{t_{j+1}, T}^{v^{k+1}}(y+\kappa)
$$

4. Calculate for $j=0$ to $j=N-1$ the mapping $\phi_{t_{j+1}, 0}^{k+1}(y)$ using:

$$
\phi^{v_{t_{j}, 0}^{k+1}}(y)=\phi_{t_{j-1}, 0}^{v^{k+1}}(y-\kappa)
$$

5. Calculate for $j=N-1$ to $j=0$ the image $J_{t_{j}}^{0}=I_{m} \circ \phi_{t_{j}, 0}^{k+1}$.

6. Calculate for $j=0$ to $j=N-1$ the image $J_{t_{j}}^{1}=I_{f} \circ \phi_{t_{j}, T}^{k+1}$.

7. Calculate for $j=0$ to $j=N-1$ the gradient of the image $\nabla J_{t_{j}}^{0}=\left[\frac{\partial J^{0}}{\partial x} \frac{\partial J^{0}}{\partial y}\right]^{\prime}$.

8. Calculate for $j=0$ to $j=N-1$ the Jacobian of the transformation $\left|D \phi_{t_{j}}\right|$. 
9. Calculate for $j=0$ to $j=N-1$ the gradient $\nabla_{v^{k+1}} E$ for $v^{k+1}$ using

$$
\left(\nabla_{v^{k+1}} E_{t}\right)_{V}=2 v_{t}^{k+1}-K\left(\frac{2}{\sigma^{2}}\left|D \phi_{t, 1}^{v^{k+1}}\right| \nabla J_{t}^{0}\left(J_{t}^{0}-J_{t}^{1}\right)\right)
$$

10. Calculate the norm of the new gradient ||$\nabla_{v^{k+1}} E \|$, Stop if below the threshold.

$$
\left\|\nabla_{v^{k+1}} E\right\|=\sum_{j=1}^{N-1}\left\|\nabla_{v_{j}^{k+1}} E\right\|
$$

11. Calculate the new energy using

$$
E\left(v^{k+1}\right)=\sum_{j=0}^{N-1}\left\|v_{t_{j}}^{k+1}\right\|_{V}^{2} \delta t+\frac{1}{N_{1} N_{2} N_{3}} \sum_{y \in \Omega}\left|J_{T}^{0}(y)-J_{T}^{T}(y)\right|^{2} .
$$

12. Re-iterate $k=k+1$, until maximum iteration reached.

Post Processing and gather results:

1. Compute the final velocity field $\hat{v}$ :

$$
\hat{v}=\arg \inf _{v \in L^{2}([0,1], V)} E(v) \doteq \int_{0}^{1}\left\|v_{t}\right\|_{V}^{2} d t+\frac{1}{\sigma^{2}}\left\|I_{m} \circ \phi_{1,0}^{v}-I_{f}\right\|_{L^{2}}^{2}
$$

2. Calculate length of the path on the manifold (geodesic distance):

$$
\operatorname{Length}\left(I d, \phi_{T}^{v^{k}}\right)=\sum_{j=0}^{N-1}\left\|v_{t_{j}}^{k}\right\|_{V} \delta t
$$

Note that in the LDDMM algorithm, the operator $L$ requires special attention to ensure a diffeomorphism, which is also chosen to be implemented in the Fourier domain for easy computation. It is given by

$$
L=-\alpha \nabla^{2}+\gamma I ; \text { where } \nabla^{2}=\frac{\partial^{2}}{\partial^{2} x}+\frac{\partial^{2}}{\partial^{2} y} .
$$


For image registration, $L$, the self-adjoint operator chosen to be of the Cauchy-Navier type and implemented as a discrete kernel:

$$
L=-\alpha\left[\begin{array}{ccc}
0 & 1 & 0 \\
1 & -4 & 1 \\
0 & 1 & 0
\end{array}\right]+\gamma\left[\begin{array}{lll}
0 & 0 & 0 \\
0 & 1 & 0 \\
0 & 0 & 0
\end{array}\right]=\left[\begin{array}{ccc}
0 & -\alpha & 0 \\
-\alpha & 4+\gamma & -\alpha \\
0 & -\alpha & 0
\end{array}\right]
$$

Thus, the kernel $K$ can be constructed as: $K=\left(L^{\dagger} L\right)^{-1}$. Since $L=L^{\dagger}, K=\left(L^{2}\right)^{-1}$. The Fourier transform can then be taken on both sides in: $A^{2}(K) F(K)=G(K)$. As a result, $F(K)=G(K) / A^{2}(K)$, where

- $G(K)$ is the Fast Fourier transform (FFT) of $g$, which is a complex conjugate image;

- $A(K)$ is the FFT of $L$. We first create a zero real image $\tilde{L}$ of size $a \times b$, then tile the kernel form of $L$ at the center of $\tilde{L}$. Take FFT of $\tilde{L}$ and obtain $A(K)$, which is a complex conjugate image;

- $F(K)$ is the Fourier transform of $f . F(K)$ is obtained by complex number division of $F(K)_{x, y}=\frac{G(K)_{x, y}}{A(K)_{x, y} \times A(K)_{x, y}} . F(K)$ is a complex conjugate image;

- $f$ is obtained by taking iFFT of $F(K)$, which is a real image.

Although demonstrating a solid mathematical foundation, the original LDDMM algorithm in [12] is proven to be highly space- and time-consuming, when the discretized time variable $t$ is sufficiently fine (usually around 20 intervals). To deal with this problem, the stationary parameterization framework [44] has been proposed to modify the original LDDMM algorithm into stationary LDDMM [49, 50], where the LDDMM registration algorithm has been greatly accelerated, without compromising its performance. Note that certain versions of LDDMM algorithm require prior information on the anatomy of the imaged object. For instance, DARTEL [50] requires segmentation of the entire brain as part of the inputs. 


\section{Chapter 3}

\section{Impact of topological changes on deformable registration algorithms}

In order to register images with topological changes, it is important to take two facts into consideration. First, given a pair of source and target images, if they differ from each other in terms of both structural and topological changes, the two types of differences will cause deformation simultaneously. Second, since the space of images and the space of their possible deformations are both of extremely high dimension, diffusion-based methods have been the most commonly used method in searching for the optimal registration [39, 12, 13, 41]. Due to these two facts, if not handled properly, all discrepancies between the pair of images, including topological changes, will drive the gradient descent process when searching for the registration solution. If this is the case, the resulting registration will be a mixture of deformations caused by image structural differences and intensity differences. In this chapter, we take the diffeomorphic demons and the LDDMM algorithms as examples to demonstrate the impact of topological changes on deformable registration. We also design a simple inpainting-based tool as a pilot study to show the possibility of removing false deformation in targeted regions. 


\subsection{Impact of topological changes on deformable reg- istration methods}

Among state-of-the-art registration algorithms, e.g., demons [13, 41] and LDDMM [12, 49], the evolution of the deformation field is achieved by the minimization of an objective energy function, as described in Chapter 2. This minimization is performed using gradient descent, an iterative process that is driven by a PDE. In these works, inverse consistency (the resulting deformation field will remain the same if the source and the target images are swapped) and diffeomorphism (both the deformation field and its inverse field are continuous and differentiable) are the two commonly imposed constraints. With these two constraints, the energy function of demons is defined as [51],

$$
E_{\text {demons }}=\operatorname{Sim}\left(I_{f}, I_{m}, \phi\right)+\frac{1}{\sigma_{T}^{2}} \operatorname{Reg}(\phi)
$$

and that of LDDMM is given by [51],

$$
E_{\mathrm{LDDMM}}=\operatorname{Sim}_{L^{2}}\left(I_{f}, I_{m}, \phi\right)+\int_{0}^{1}\left\|v_{t}\right\|_{V}^{2} d t
$$

Note that, in both equations, the first term is a symmetric image similarity measure, given by $\operatorname{Sim}\left(I_{f}, I_{m}, \phi\right)=\frac{1}{2}\left(\operatorname{Sim}\left(I_{f}, I_{m} \circ \phi^{-1}\right)+\operatorname{Sim}\left(I_{m}, I_{f} \circ \phi\right)\right)$, which ensures the inverse consistency. The second term is a regularization term to ensure the diffeomorphism. In these two works, diffeomorphism is realized differently. Specifically, in demons, a Gaussian kernel is used on the deformation field or the update of the deformation field, resulting in diffusion-like or fluid-like regularization, respectively, and a deformation field composition is used in the updating rule. In LDDMM, diffeomorphism is achieved by confining the deformation within a Lie group of diffeomorphisms using a differential operator $L$.

When registering images of healthy subjects, both inverse consistency and diffeomorphism are important constraints to ensure that the results of the registration algorithm are mathematically stable and physically realistic. However, both of them interact with topological changes and thus cause problems during the registration process. Fig. 3.1 gives a simple example to illustrate the problem. Suppose a pair of 2-D images, $I_{m}$ (Fig. 3.1(a)) and $I_{f}$ (Fig. 3.1(b)), are to be co-registered. Clearly, $I_{m}$ carries a topological change with much lower intensity. The glyph views of the registration results using diffeomorphic demons ${ }^{1}$ are

\footnotetext{
${ }^{1}$ The implementation is from http://www.insight-journal.org/browse/ publication/644, by Vercauteren et al..
} 


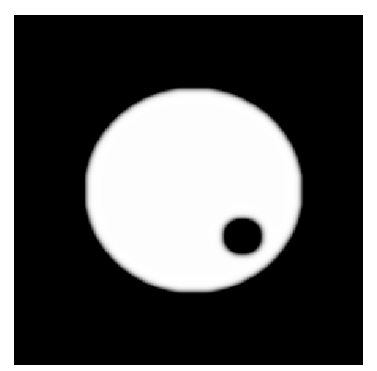

(a)

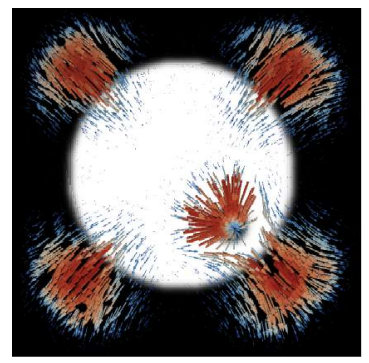

(c)

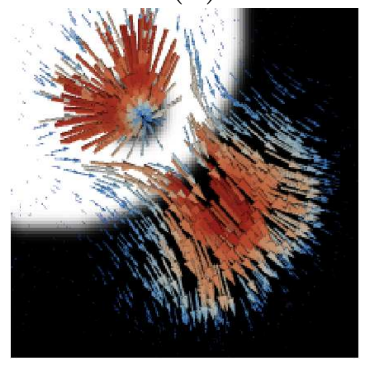

(d)

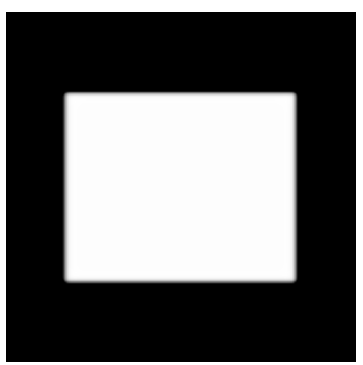

(b)

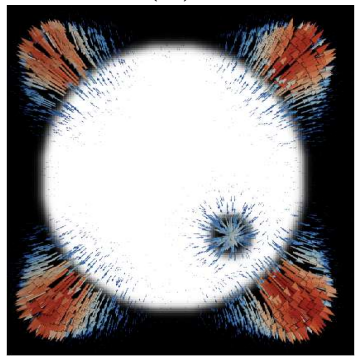

(e)

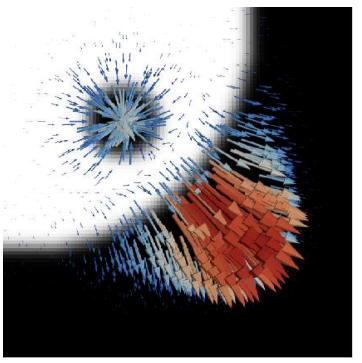

(f)

Figure 3.1: Registration of simple images with topological change. (a): moving image $I_{m}(\mathrm{~b})$ : fixed image $I_{f} ;(\mathrm{c})$ : glyph view of the deformation field generated from diffeomorphic demons; (d): zoom-in view of (c) around the region with topological change; (e): the deformation field generated from symmetric LDDMM; (f) zoom-in view of (e) around the region with topological change.

shown in Fig. 3.1(c) and (d), and those using symmetric $\mathrm{LDDMM}^{2}$ are given in Fig. 3.1(e) and (f). Note that a similar experiment has been conducted with HDW, which also demonstrates the same problem as discovered here: "pathology is ... case where the validity of the registration is compromised" [18]. These results demonstrate that with the symmetric intensity similarity measure, the intensity difference caused by topological changes forces the local neighborhood to deform in both algorithms. Note that these local deformations are toward the inside of the associated topological change, following the direction of the gradient. Therefore, they are not related to the pathological nature of the topological change, i.e., false deformations. Furthermore, given a diffeomorphism, the smoothing operations generally spread false deformation over an enlarged neighborhood.

\footnotetext{
${ }^{2}$ This is our implementation of the algorithm by Beg et al.[12], with the symmetric image similarity measure in $(3.2)$.
} 


\subsection{A simple algorithm to suppress the impact of topo- logical change through in-painting}

As discussed earlier, it is not practical to model the growth of a topological change without the prior knowledge of its pathological nature. Instead, a more general approach is to separate the regions affected by topological changes, and perform the registration without the impact from these regions. Therefore, the result of a registration algorithm designed in such a fashion reveals only structural changes, while a segmentation label map, as a by-product, is used to isolate the possible locations of the topological changes for further clinical study. To achieve this, we proposed a two-round registration method that can be incorporated onto any currently available iterative PDE-driven registration methods. In the following, we take symmetric LDDMM as an example to illustrate our proposed method. The proposed method does not interfere with the execution of the registration process, so using other registration algorithms, e.g., diffeomorphic demons, will not change the conclusion we draw here. In addition, because the registration algorithm we use is inverse consistent, we assume the source image $I_{m}$ is the one that contains topological change in the following discussion. In other words, the same operation can be performed on the target image $I_{f}$, if it is the one that contains topological change, by simply exchanging the forward and backward deformations.

We use simple 2-D images $I_{m}$ and $I_{f}$ shown in Fig. 3.1 (a) and (b), respectively, as inputs to explain the above algorithm. The first round of registration takes the original input images. Thus, due to the presence of topological changes, we expect that the registration algorithm will not converge to the point where the energy gradient decreases to a sufficiently small value. Instead, we force the registration to terminate when the energy gradient stops decreasing. Because of this, we refer to this round of registration as coarse registration. We can examine how image dissimilarity drops over iterations to model the evolution of the coarse registration process. Specifically, we compute an absolute image residual Diff $k$ at iteration $k$, and sum up these image residuals from all the iterations during the coarse registration process to obtain a total difference Diff $t$ as shown in Fig. 3.2(a). Note that, all the absolute image residuals are in the image space of the target image $I_{f}$, we thus need to back propagate them into the image space of $I_{m}$ by applying their corresponding deformation $\phi_{k}$. From Fig. 3.2(a), we can clearly observe that regions with intensity disagreement between $I_{m}$ and $I_{f}$, which are due to structural differences, gradually shrink as the registration process proceeds. Hence, these regions appear only in some of the Diff ${ }_{k} \mathrm{~s}$ and consequently have a low 


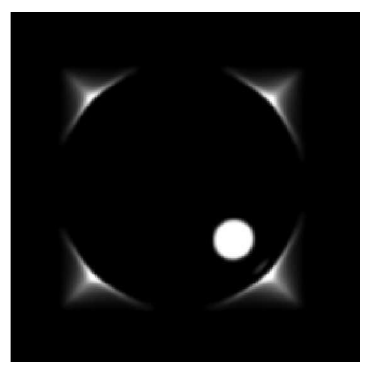

(a)

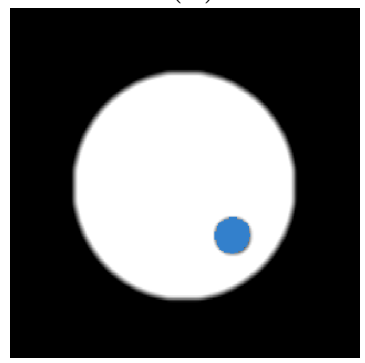

(e)

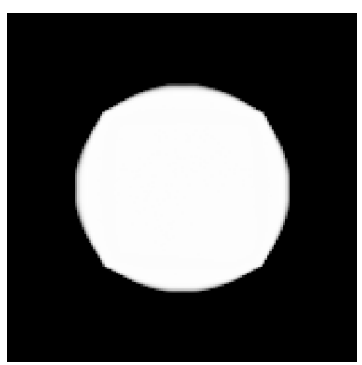

(b)

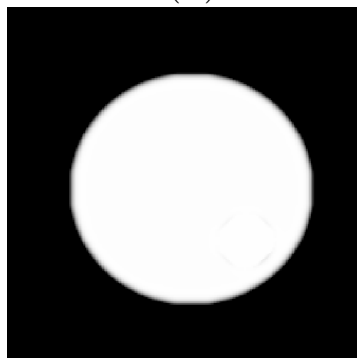

(f)

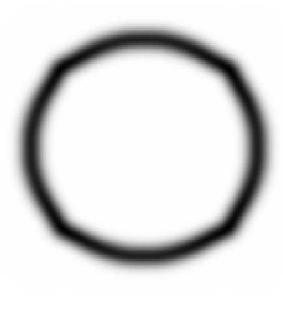

(c)

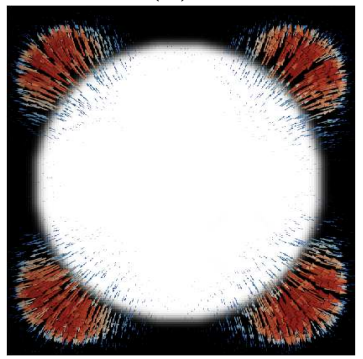

$(\mathrm{g})$

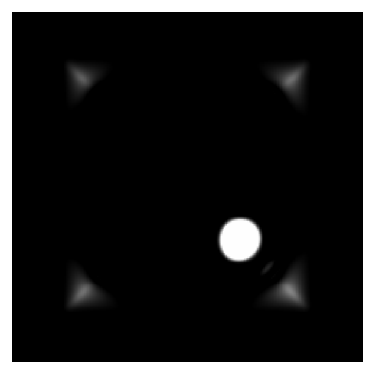

(d)

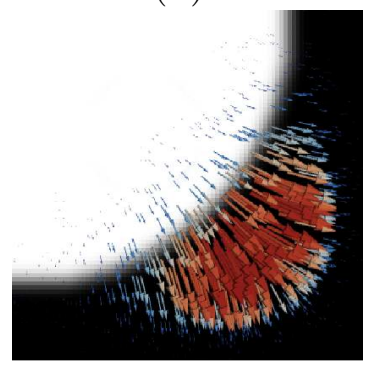

(h)

Figure 3.2: Illustration of our registration method. (a): total difference Diff $t$ obtained after the coarse registration; (b): deformed $I_{1}$ into the image space of $I_{m} ;(\mathrm{c})$ : the edge map GM of (b); (d): the probability map $P$ of topological changes in $I_{m} ;($ e): binary label map of topological changes in $I_{m}$; (f): $I_{m}^{\prime}$ obtained after repairing; (g): the deformation field obtained after the fine registration; (h): the zoom-in view of (g) around the topological change in $I_{m}$.

intensity in Diff. On the other hand, those intensity disagreements caused by topological changes persist throughout the deformation process and thus accumulate in Diff $t$. Other sources of image disagreement that also get accumulated during the registration process include the interpolation error and local mismatch caused by smoothness constraints. These disagreements happen to the regions along object edges in $I_{f}$. To eliminate them, we deform $I_{f}$ into the image space of $I_{m}$ in Fig. 3.2(b) and compute its edge map GM $=1-\nabla\left(I_{f} \circ \phi_{n}\right) * K$ given in Fig. 3.2(c), where $K$ is a Gaussian smoothing kernel with a variance that is enough to spread the edge over the mismatched band. By performing a pixel-wise multiplication $\otimes$ between the normalized Diff $t$ and GM, we obtain a probability map P shown in Fig.3.2(d) of the topological changes in $I_{m}$. We threshold P (a threshold of 0.5 is used in both our 2-D and 3-D registration examples), followed by 1 to 2 rounds of 8-neighborhood dilation, to obtain a binary label map (blue region in Fig. 3.2(e)) which indicates the topological change in $I_{0}$. The region corresponding to the topological change is then replaced by an interpolation 
of the intensity of pixels in its neighborhood. This operation therefore provides us with a repaired image $I_{m}^{\prime}$ given by Fig. 3.2(f). $I_{m}^{\prime}$ and $I_{f}$ are then taken as inputs to the deformable registration algorithm with normal stopping criteria, which generates the final deformation fields $\phi$ and $\phi^{-1}$. The resulting $\phi$ is shown in Fig. 3.2(g) and (h). By comparing these results to those in Fig. 3.1(c)-(e), we observe that the false deformation caused by the topological change has been eliminated.

\subsection{Results}

Our proposed method can be summarized in the following algorithm:

\section{Algorithm: repairing lesion in deformable registration:}

Initialize total difference $\operatorname{Diff}_{t}=0$

Coarse registration iteration $k$ :

- compute gradient of energy function: $\nabla E$;

- update deformation field and its inverse: $\phi_{k}$ and $\phi_{k}^{-1}$;

- compute absolute image difference:

$\operatorname{Diff}_{k}=\left|I_{m} \circ \phi_{k}^{-1}-I_{f}\right|$;

- back propagate $\operatorname{Diff}_{k}$ and update total difference:

$\operatorname{Diff}_{t}+=\operatorname{Diff}_{k} \circ \phi_{k}$

- update energy function: $E$;

Coarse registration is forced to terminate with a loose stopping criteria after $n$ iterations with the resulting deformation fields $\phi_{n}$ and $\phi_{n}^{-1}$;

Coarse registration post-processing : repair $I_{m}$

- compute gradient magnitude of deformed target image:

$\mathrm{GM}=1-\nabla\left(I_{f} \circ \phi_{n}\right) * K$

- normalize GM and Diff $t$ to $[0,1]$;

- compute probability map of topological changes: $\mathrm{P}=\operatorname{Diff}_{t} \otimes \mathrm{GM}$ 


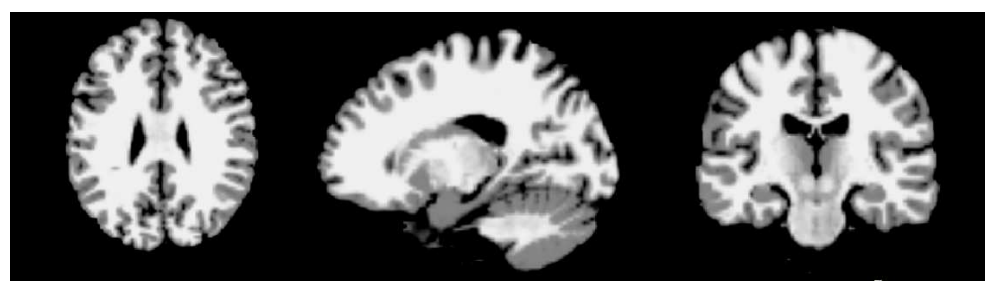

(a)

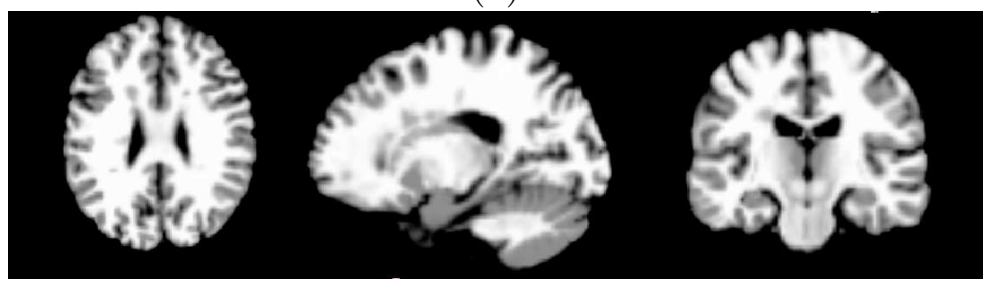

(b)

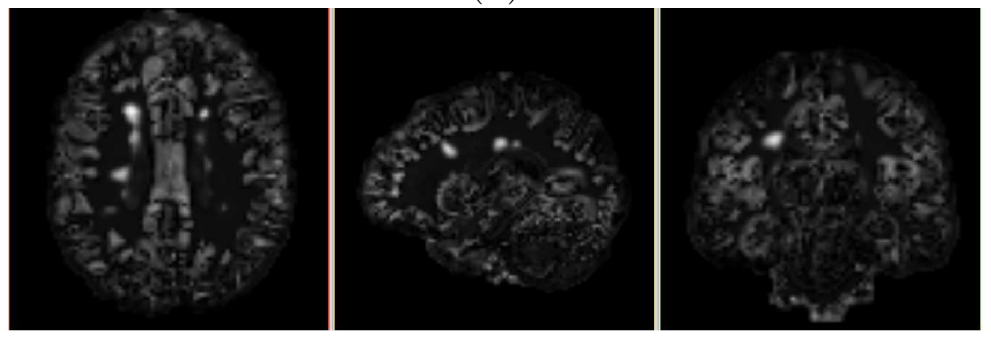

(c)

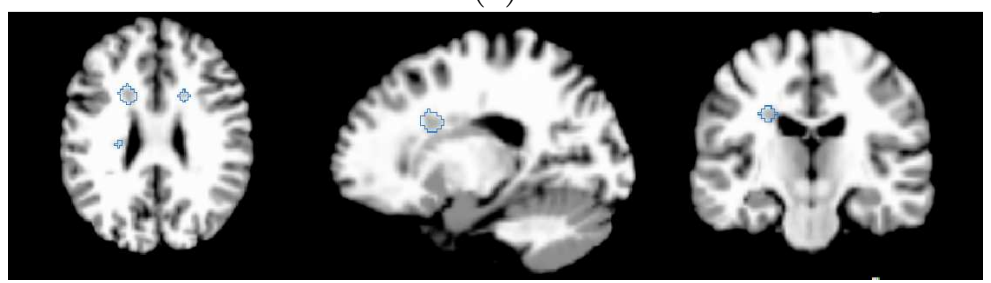

(d)

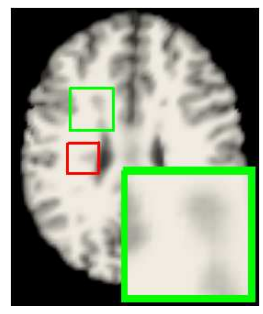

(e)

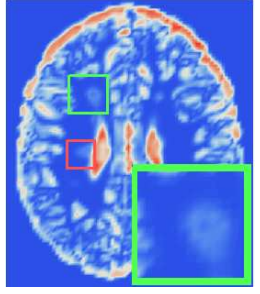

(f)

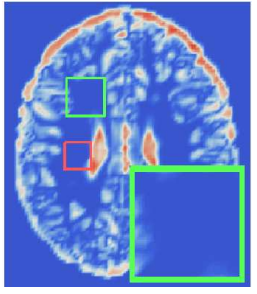

$(\mathrm{g})$

Figure 3.3: Registration results of brain MRIs with MS, using our proposed method. (a): target image $I_{f}$; (b): source image $I_{m}$ which contains MS and after a nonlinear deformation from $I_{f}$; (c): probability map of topological change P; (d): binary map of detected lesion in $I_{m}$; (e): an axial slice of $I_{m} ;(\mathrm{f})$ and $(\mathrm{g})$ : the magnitude of the deformation field at the same slice as in (e), after the coarse and fine registrations, respectively. Inside the green boxes: regions with MS lesions. Regions within green boxes are enlarged at the bottom. 
- threshold P followed by a slight dilation, to obtain a binary label map of topological changes;

- segmented topological changes are interpolated using the intensity values of their neighborhood, leading to a repaired version of the input image $I_{m}^{\prime}$.

Fine registration replace $I_{m}$ by its repaired version $I_{m}^{\prime}$ to re-perform registration with normal stopping criteria, and output deformation field $\phi$ and $\phi^{-1}$.

This in-painting-based simple method is used to register two 3-D image volumes, where one of them carries simulated MS lesions. These image volumes are obtained from the McConnell Brain Imaging Center Simulated Brain Database [52]. The Axial, Coronal and Sagittal views of the healthy image volume are shown in Fig. 3.3(a), and we use it as the target image $I_{f}$. To generate source image $I_{0}$, simulated MS lesion is introduced into $I_{f}[52]$, followed by a nonlinear $3-\mathrm{D}$ shape distortion, to simulate brain structural change. $I_{m}$ is shown in Fig. 3.3(b).

We perform a coarse registration to obtain the total difference Diff $t$, which is then multiplied by the edge map of deformed $I_{f}$ to obtain the probability map of lesion $\mathrm{P}$, as shown in Fig. 3.3(c). $\mathrm{P}$ is then thresholded at the mid-level, i.e., 0.5, and dilated for two rounds to obtain a binary label map of possible lesions in $I_{m}$. This label map is shown as blue contours in Fig. 3.3(d). From Fig. 3.3(d), we notice that our method is able to identify major MS lesions in $I_{0}$. However, several lesions, which have smaller intensity differences from the white matter, are not successfully identified (can be seen from the axial image). We repair the identified lesion regions via interpolating the intensity of their local neighborhoods, and reregister the images to obtain a final deformation field. Fig. 3.3(e)-(g) provides a comparison between the registration fields before and after lesion-repairing, where the regions within the green boxes are enlarged at the bottom-right corners for better visualization. Fig. 3.3(f) and (g) are color coded magnitude of the deformation fields after the coarse and fine registrations, respectively, and they are of the same image slice as in Fig. 3.3(e). We can observe that the regions inside the green and red boxes in Fig. 3.3(f) contain false deformation caused by the lesions, whereas these false deformations are successfully removed from the deformation field of the fine registration, as shown in Fig. $3.3(\mathrm{~g})$.

This simple method, as well as its registration results, demonstrates that it is necessary to specially handle topological changes in deformable registration algorithms. Despite the efficacy of our proposed method, two major aspects require some improvement. First, the simple in-painting used above requires a relatively accurate segmentation of lesion. However, 
clinical data usually have poorly defined lesion boundaries, where a precise segmentation can be difficult to obtain. Second, in practical situations, lesion can cover anatomical regions with different intensity, for example, MS lesion usually covers areas where white matter appears to be darker and joins the ventricle. In these cases, in-painting will cause a noticeable artifact. These sharp boundaries and artifacts are essentially the same as topological changes, which will cause false deformation in the registration. To illustrate this, two samples of simple in-painting results of lesion segmented by FreeSurfer are given in Fig. 3.4 (a) and (b). In both subfigures, the first row shows the brain MR image with a severe lesion. The direct in-painting repaired result is given in the second row, where the artifacts are noticeable alone the lesion segmentation boundaries. In addition, merely for comparison purposes, in the third row we include the desired lesion repair results, which are obtained using our deformable registration algorithm that will be discussed in Chapter 4. From these figures, the lesion repair result is apparently improved by using our deformable registration algorithm. 

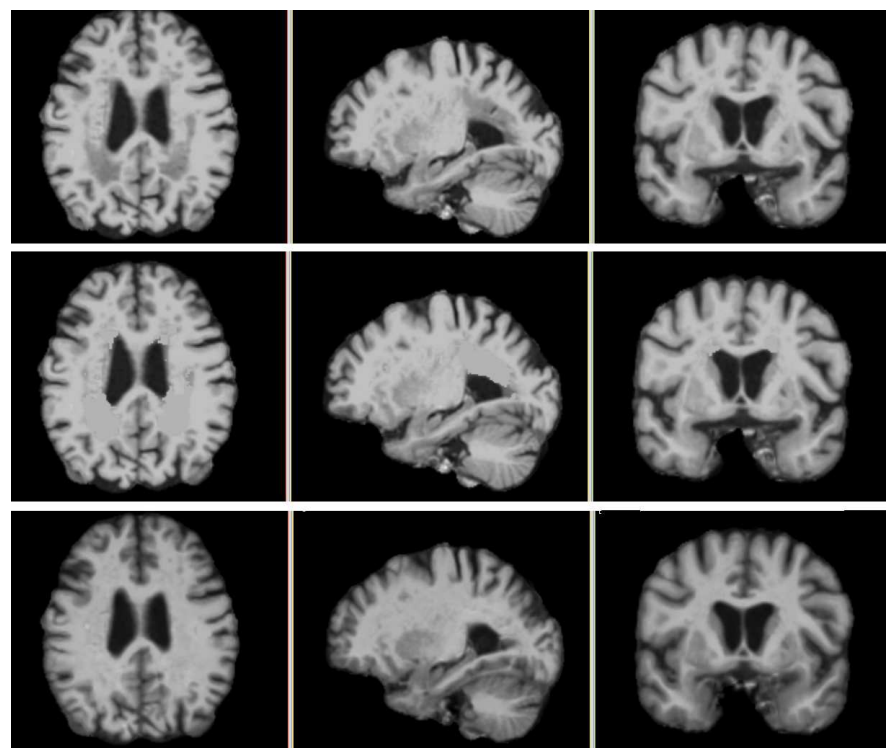

(a)
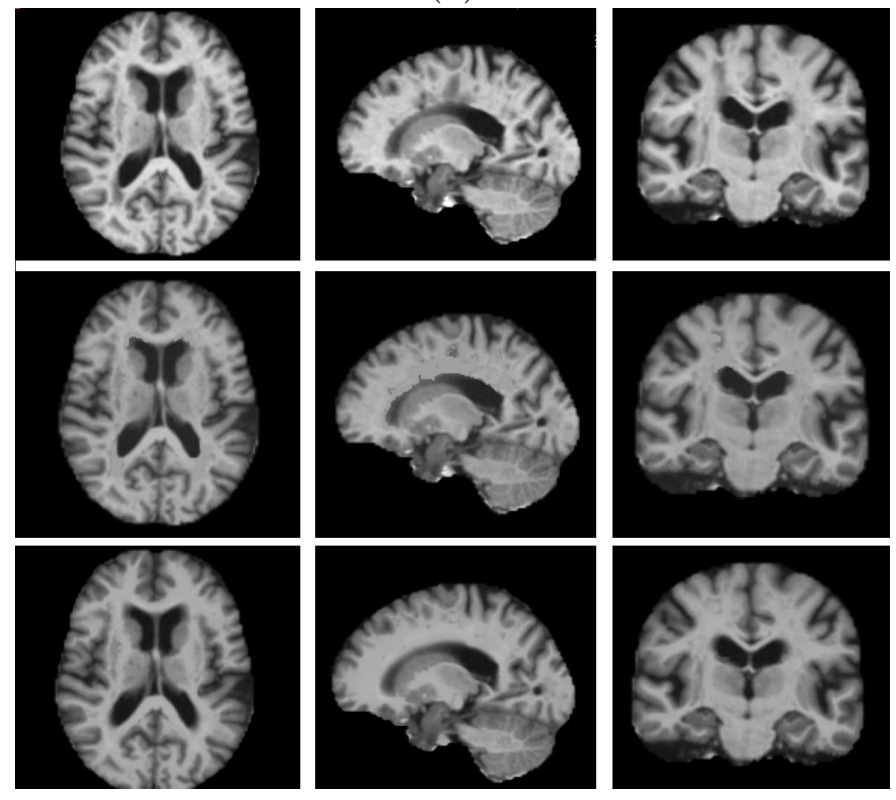

(b)

Figure 3.4: Samples of in-painting results of lesion segmented by FreeSurfer. (a) and (b): each gives Axial, Sagittal and Coronal views of one in-painting example. In both (a) and (b), the first row shows the brain MR image with severe MS lesion. The direct in-painting repaired results are given in the second row. In addition, the third row shows the desired lesion repair results, obtained using our deformable registration algorithm presented next in Chapter 4. 


\section{Chapter 4}

\section{Registration of Images with Varying Topology using Embedded Maps}

In this chapter, we describe a new registration method to handle topological changes by suppressing their impact on the deformable registration process for brain MRI. Specifically, images in $\mathbb{R}^{3}$ Euclidian space are embedded as surfaces in an $\mathbb{R}^{4}$ Riemannian space. The registration process is then conducted as surface deformation, where the first three dimensions of the resulting deformation field correspond to the spatial grid deformation in the $\mathbb{R}^{3}$ Euclidian space, and the 4 th dimension corresponds to the intensity displacement. The contribution includes two aspects. First, compared with metamorphosis, our embedding models the two types of deformation in a metamorphosis, i.e., a spatial deformation and a template evolution, with a single partial differential equation (PDE) evolution in a higher dimensional space. This guarantees a smooth convergence of the diffusion to a local minimum (refer to Sec. 4.2.2). Second and more importantly, by carefully choosing a scale-space in the embedding, we are able to control the distribution of the deformation energy in a way that topological changes are mainly attributed to intensity displacement, while brain structural changes are mostly captured by spatial grid deformation. In doing so, topological changes will not impact the spatial deformation and thus false deformation is effectively suppressed (refer to Sec. 4.2.1). The resulting spatial deformation is a diffemorphism, evolved using the intrinsic update step, as proposed in [13]. 


\subsection{Preliminaries}

Our algorithm is derived from the Sochen-Kimmel-Malladi general non-linear diffusion [1], by choosing a particular embedding for our specific problem. The surface evolution follows the Euler-Lagrange equation that minimizes the surface weight measured by the Polyakov functional. Specifically, an image manifold $(\Sigma, g)$ in $\mathbb{R}^{3}$ is embedded as an $\mathbb{R}^{4}$ feature-space manifold $(M, h)$, where $g$ and $h$ are the positive definite symmetric bilinear forms defined on $\Sigma$ and $M$, respectively. In Riemannian geometry, $g$ and $h$ are called metrics, which defines an inner product on the corresponding manifold and are used to measure distances on the surfaces. In the embedding, the map $X: \Sigma \rightarrow M$ is chosen as

$$
X=[x, y, z, I(x, y, z)],
$$

where $x, y, z$ are the local image Cartesian coordinates in $\mathbb{R}^{3}$, and $I(x, y, z)$ is the intensity of the pixel at $(x, y, z)$. A exemplar embedding from a $2 \mathrm{D}$ image manifold to a $3 \mathrm{D}$ feature-space manifold is illustrated in Fig. 4.1.

We have the freedom of choosing a particular metric $h$ on the embedded surface $M$ to achieve a desired surface weight measurement (refer to Sec 4.2). Based on the chosen map $X$ and $h$, the metric $g$ on the image manifold $\Sigma$ can be uniquely constructed. This is called the pullback procedure, and is given in the following,

$$
g_{\mu \nu}=h_{i j} \partial_{\mu} X^{i} \partial_{\nu} X^{j}
$$

Note that (4.2) uses Einstein summation convention, with $\mu, \nu \in\{x, y, z\}, i, j \in\{x, y, z, I\}$ in our embedding. The summation convention will be used in all our equations from now on. In $(4.2), g_{\mu \nu}$ represents the $(\mu, \nu)$ th element of $g$ and $h_{i j}$ stands for the $(i, j)$ th element of $h$, where both $g$ and $h$ are in matrix form.

Using the above definitions, the weight of the map $X: \Sigma \rightarrow M$ is calculated as:

$$
S\left[X^{i}, g_{\mu \nu}, h_{i j}\right]=\int d^{m} \sigma \sqrt{g} g^{\mu \nu} \partial_{\mu} X^{i} \partial_{\nu} X^{j} h_{i j}
$$

where $g^{\mu \nu}$ is the $(\mu, \nu)$ element of $g^{-1}, \sqrt{g}$ is the square root of the metric $g$ 's determinant, and $m$ is the dimension of $\Sigma$. This equation is referred to as the Polyakov functional in Riemannian geometry. The variation of the Polyakov functional with respect to the embedding can be found by the Euler-Lagrange equation,

$$
-\frac{1}{2 \sqrt{g}} h^{i j} \frac{\delta S}{\delta X^{l}}=\frac{1}{\sqrt{g}} \partial_{\mu}\left(\sqrt{g} g^{\mu \nu} \partial_{\nu} X^{k}\right)+\Gamma_{i j}^{k} \partial_{\mu} X^{i} \partial_{\mu} X^{j} g^{\mu \nu}
$$




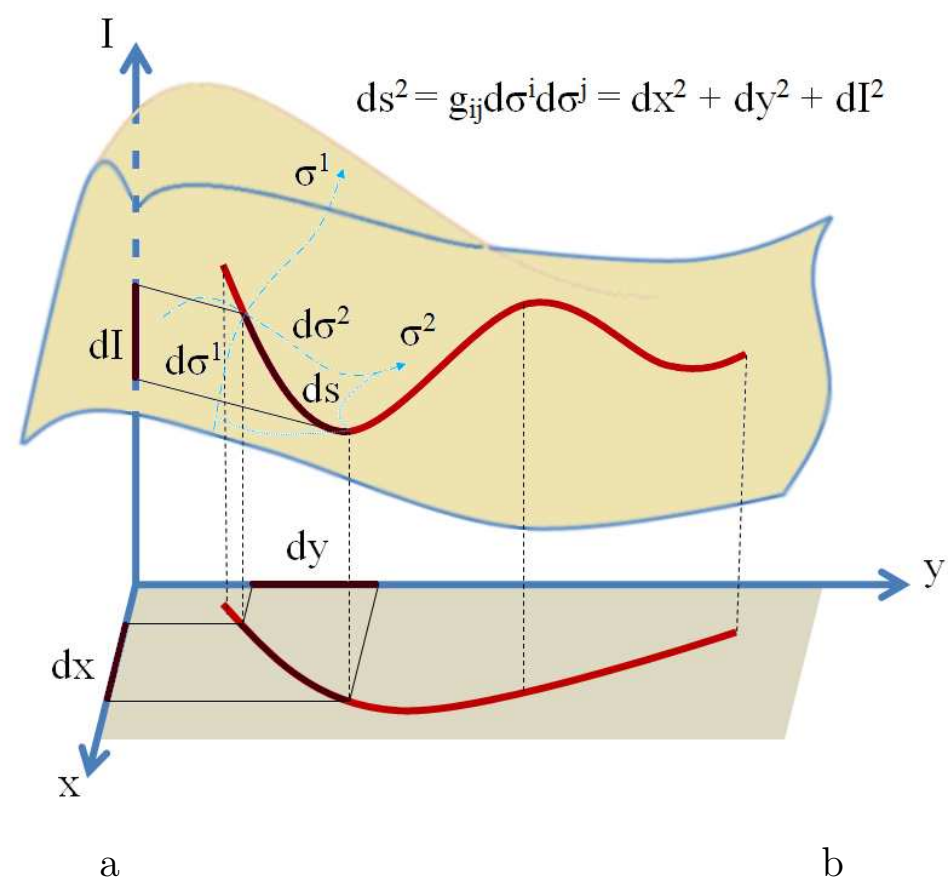

Figure 4.1: Embedding and the length element [1]. A 2D image is embedded into a 3D surface, where the third dimension is the image intensity $I$. The length element $d s$ on the embedded surface is to be computed via the metric $g$.

where $\Gamma_{i j}^{k}, k, i, j \in\{x, y, z, I\}$ are the elements in the Levi-Civita connection. In Riemannian geometry, a connection describes how the frame is changed infinitesimally on the manifold. A connection is said to be metric compatible if the covariant derivatives of the metric on the manifold vanish. Thus, the definition of the Levi-Civita connection is given by [1]:

The fundamental theorem of Riemannian geometry: on a Riemannian manifold $(M, g)$ there exists a unique symmetric connection which is compatible with the metric $g$. This connection is called the Levi-Civita Connection and the components are given by the following expression:

$$
\Gamma_{j k}^{i}=\frac{1}{2} h^{i l}\left(\partial_{j} h_{l k}+\partial_{k} h_{j l}-\partial_{l} h_{j k}\right) .
$$

Note that, in Equation (4.4) the factor multiplied before $\frac{\delta S}{\delta X^{l}}$ is to simplify the mathematical expression and does not change the minimization solution. Equation (4.4) defines the gradient descent direction that minimizes the Polyakov functional, following which the embedded surface shrinks its area most rapidly. Therefore, (4.4) is referred to as the minimal 
surface flow. It has been shown that many image processing tasks can be accomplished via minimizing the Polyakov functional, e.g., image smoothing and segmentation [1].

\subsection{Registration method}

In this chapter, we describe a new registration algorithm in the same spirit, i.e., via the minimization of the Polyakov Functional. This algorithm differs from existing methods in the sense that it uses a segmentation of the topological changes as an input and is capable of registering brain MRI, while eliminating the false deformation. The following describes the three major components of the proposed algorithm and then summarizes them into a registration algorithm.

\subsubsection{Choice of embedding}

To establish a registration objective, we define the metric $h$ to have the following form,

$$
h=\left[\begin{array}{cccc}
\phi & 0 & 0 & 0 \\
0 & \phi & 0 & 0 \\
0 & 0 & \phi & 0 \\
0 & 0 & 0 & \beta \phi
\end{array}\right],
$$

where $\phi$ is a positive function that influences the shape of a harmonic map, i.e., it changes the physical meaning of a minimal weight of the surface. $\phi$ provides us with the flexibility of adjusting the path of diffusion, similar to the geodesic surfaces [53], and enables the minimal surface flow to achieve the desired objective. $\beta$ defines the scale-space of the embedding, which is the relative magnitude between feature and space, i.e., image intensity and image spatial Cartesian grid.

Taking inspiration from geodesic active contours [54], we note that for a specific choice of metric, the Polyakov action measures the area of the surface in the space M. This gives us the freedom to use the target image of the registration to define the metric and thus influence the shape of the harmonic map. In this work, we choose $\phi=\left(I_{m}-I_{f}\right)^{2}$, where $I_{m}$ and $I_{f}$ are the moving and the fixed (target) images, respectively. The squared image difference given by $\phi$ is the most commonly used error measurement in intensity-based registration works. The difference is, in this work $\phi$ is not directly minimized; instead, $\phi$ is used to modulate the metric $h$. Intuitively, within a specific infinitesimal region on the embedded 


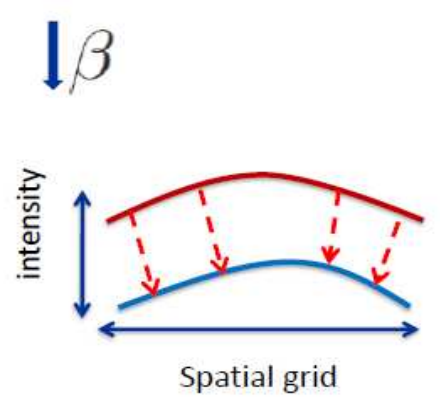

a

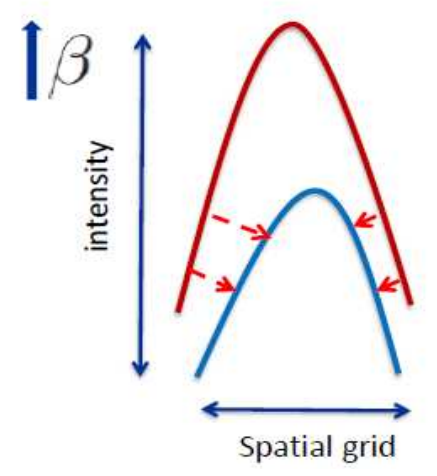

$\mathrm{b}$

Figure 4.2: Influence of $\beta$ on the deformation field.

surface, if the value of $\phi$ function decrease its value, the distance measure within this region will decrease, and the surface area measure will also decrease. Thus the physical meaning of the Polyakov function is no longer same as the commonly used surface area in the Euclidian space. Especially, when the moving and the fixed images are perfectly aligned, $\phi$ will have minimal-value everywhere and the surface area measured by the Polyakov functional will achieve its minimal value. In other words, during the diffusion, the surface area is attracted by a potential well formed by $\phi$, and our particular choice of $\phi$ transforms the Polyakov functional into a registration objective.

The factor $\beta$ defines the relative magnitude between the feature and the geometric space. The choice of $\beta$ impacts the distribution of the deformation energy. This is illustrated in Fig. 4.2, where we use red and blue curves to represent surface cut profiles of a moving and a target image, respectively and the arrows denote gradient directions of evolving from the moving image to the target image. In Fig. 4.2(a), $\beta$ is set to a small value, and thus image intensity weight less in the pullback. When the moving image is evolved to the target image, the gradient direction will be better aligned with the intensity axis, and the deformation energy will be concentrated on intensity displacement. Alternatively as shown in Fig. 4.2(b), if $\beta$ is set to a large value, the gradient direction evolving the moving image to the target image will be better aligned with the spatial grid axis, and the deformation energy will be more distributed to the spatial deformation. Based on this intuition, if we have a function $p(x, y, z)$, whose value indicates the probability that point $(x, y, z)$ lies within topological 
changes, we can construct the function $\beta$ in the following form,

$$
\beta(x, y, z)=\frac{1}{p(x, y, z)+\epsilon}
$$

where $\epsilon$ is a small positive value to avoid the ill-condition of division-by-zero. With this function, for image regions without topological change, $p(x, y, z)$ will have small values and $\beta$ will have large values accordingly. Then the deformation in these regions will mainly concentrate on spatial grid to capture the structural difference between the moving and the target images. On the other hand, for image regions that do have topological change, $p(x, y, z)$ will have large values and $\beta$ will thus have small values. Then intensity displacement will be favored during the registration process to correct the appearance of topological changes in these regions.

\subsubsection{Computation of surface variation}

Using the map given in (4.1) and the chosen $h$, we can obtain the metric $g$ through the pullback procedure,

$$
g=\phi\left[\begin{array}{ccc}
1+\beta I_{x}^{2} & \beta I_{x} I_{y} & \beta I_{x} I_{z} \\
\beta I_{x} I_{y} & 1+\beta I_{y}^{2} & \beta I_{y} I_{z} \\
\beta I_{x} I_{z} & \beta I_{x} I_{z} & 1+\beta I_{z}^{2}
\end{array}\right]
$$

Then, the gradient direction that minimizes surface area is given by the Euler-Lagrange equation (4.4). We take the gradient descent approach to obtain the equation for surface variation,

$$
\frac{\partial X}{\partial t}=\frac{\phi^{2}}{\sqrt{g}} \partial_{\mu}\left(\sqrt{g} g^{\mu \nu} \partial_{\nu} X^{k}\right)+\phi^{2} \Gamma_{i j}^{k} \partial_{\mu} X^{i} \partial_{\mu} X^{j} g^{\mu \nu}
$$

Here, $t$ is the time variable that represents a step in the diffusion process. Note that in (4.9), the Euler-Lagrange equation is multiplied by $\phi^{2}$ which is a positive function and will not affect the solution of the minimization.

Combining all the above definitions, we can obtain the registration updating rule:

$$
\frac{\partial X}{\partial t}=S_{1}\left[\begin{array}{c}
-\beta I_{x} \\
-\beta I_{y} \\
-\beta I_{z} \\
1
\end{array}\right]+S_{2}\left[\begin{array}{c}
I_{x} \\
I_{y} \\
I_{z} \\
I_{x}^{2}+I_{y}^{2}+I_{z}^{2}
\end{array}\right]+S_{3}\left[\begin{array}{c}
-\beta I_{x} \\
-\beta I_{y} \\
-\beta I_{z} \\
1
\end{array}\right]
$$


where,

$$
\begin{aligned}
S_{1}= & \frac{\phi}{\kappa^{2}}\left[\left(1+\beta I_{y}^{2}+\beta I_{z}^{2}\right) I_{x x}+\left(1+\beta I_{x}^{2}+\beta I_{z}^{2}\right) I_{y y}+\left(1+\beta I_{x}^{2}+\beta I_{y}^{2}\right) I_{z z}\right] \\
& -\frac{2 \phi \beta}{\kappa^{2}}\left(I_{x} I_{y} I_{x y}+I_{x} I_{z} I_{x z}+I_{y} I_{z} I_{y z}\right)+\frac{3}{2 \kappa}\left(\phi_{x} \beta I_{x}+\phi_{y} \beta I_{y}+\phi_{z} \beta I_{z}-\phi_{I}\right), \\
S_{2}= & -\frac{\phi_{I}}{2 \kappa}, \\
S_{3}= & -\frac{\phi}{2 \kappa \beta}\left(\beta_{x} I_{x}+\beta_{y} I_{y}+\beta_{z} I_{z}\right)(1+\kappa), \\
\kappa= & 1+\beta I_{x}^{2}+\beta I_{y}^{2}+\beta I_{z}^{2},
\end{aligned}
$$

Note that in (4.10), we intentionally assemble all the terms that are related to the partial derivatives of $\beta$ in $S_{3}$. The first three dimensions in (4.10) define the spatial grid deformation, i.e., the structural differences between the moving and the target images. The 4th dimension in (4.10) defines the intensity displacement, which is mainly concentrated on the pre-identified topological changes. The detailed derivation of (4.10) can be found in Appendix A. We want to emphasize that when using (4.10), the distribution of the deformation energy in the intensity displacement and the spatial grid deformation can be directly controlled by the function $\beta$. This is the major reason that false deformation can be eliminated from our resulting deformation field. To better understand this, if we set $\beta$ to extreme values of $+\infty$ and 0 , we can obtain the following two equations (for details refer to Appendix B),

$$
\lim _{\beta \rightarrow+\infty} \frac{\partial X}{\partial t}=\left[\begin{array}{llll}
I_{x} H & I_{y} H & I_{z} H & 0
\end{array}\right]^{\prime}
$$

where

$$
\begin{aligned}
H= & -\frac{\phi I_{x}\left[\left(I_{y}^{2}+I_{z}^{2}\right) I_{x x}+\left(I_{x}^{2}+I_{z}^{2}\right) I_{y y}+\left(I_{x}^{2}+I_{y}^{2}\right) I_{z z}-2\left(I_{x} I_{y} I_{x y}+I_{x} I_{z} I_{x z}+I_{y} I_{z} I_{y z}\right)\right]}{\left(I_{x}^{2}+I_{y}^{2}+I_{z}^{2}\right)^{2}} \\
& -\frac{3 I_{x}\left(\phi_{x} I_{x}+\phi_{y} I_{y}+\phi_{z} I_{z}\right)}{2\left(I_{x}^{2}+I_{y}^{2}+I_{z}^{2}\right)}
\end{aligned}
$$

and

$$
\lim _{\beta \rightarrow 0} \frac{\partial X}{\partial t}=\left[\begin{array}{llll}
I_{x} T & I_{y} T & I_{z} T & \infty
\end{array}\right]^{\prime}
$$

where

$$
T=\phi_{I} I_{x}-2 \phi I_{x}\left(\beta_{x} I_{x}+\beta_{y} I_{y}+\beta_{z} I_{z}\right) .
$$

It is easy to observe that (4.11) and (4.12) agree with our expectation (refer to Sec. 4.2.1) that when $\beta$ has very large values, the spatial grid deformation should contain the most 
energy, whereas when $\beta$ has very small values, the intensity displacement should dominate the deformation energy. Note that we do not require a precise lesion segmentation to form a proper $\beta$ function. It can be based on any estimated probability function $p(x, y, z)$. In real applications, $p(x, y, z)$ can be constructed from some lesion-likelihood measurements, or a smoothed version of a rough lesion segmentation, given by

$$
p \doteq K_{p r o b} * L,
$$

where $K_{\text {prob }}$ is a Gaussian kernel and $L$ is the segmentation label map for lesions. In most cases, after intensity displacement, the spatial deformation of lesion-affected areas will be filled by the interpolation of the deformation from their neighborhood, due to the smoothness constraints of the deformation field. Thus, when we construct $\beta$ from a smoothed rough lesion segmentation, slightly over- or under-segmentation is well tolerated.

\subsubsection{Diffeomorphism}

Most state-of-the-art registration algorithms constrain the resulting deformation field to be in the group of diffeomorphisms [13, 12]. As described in Chapter 2, a diffeomorphism is a Lie group of invertible and differentiable bijective mappings. A dense deformation field that is diffeomorphic indicates no tearing or folding in the physical space after deformation. Constraining the evolution of deformation field within the group of diffeomorphism also ensures a stable diffusion process by avoiding spikes when solving the associated PDE. A subtle difference in our registration is that, only the first three dimensions of the surface evolution represent spatial grid deformation, and thus are required to be diffeomorphic. The 4th dimension, however, is the intensity displacement that is expected to capture high frequency variations and should not be confined in the group of diffeomorphisms ${ }^{1}$.

Clearly, in our method, adding explicit constraints only to the first three dimensions of the deformation is mathematically difficult. Instead, as proposed in the work of diffeomorphic

\footnotetext{
${ }^{1}$ Ideally, the intensity displacement is expected to have certain smoothness to ensure a smooth PDE evolution. In the algorithm step 5, note that there is a Gaussian kernel directly applied on the update of intensity displacement. In all the experiments, a very small Gaussian kernel with $\sigma_{\text {int }}=0.4$ for $K_{\text {int }}$, was used and always results in a stable diffusion. However, diffeomorphism is too strong for this smoothness constraint. Imagine that, there are two pixels, left and right. If the input image has a smaller intensity on the left pixel, diffeomorphism essentially ensures that after intensity displacement, the left pixel cannot change to a larger intensity compared to the right pixel, i.e., maintaining the "ordering" of the intensities. Our algorithm does allow the flexibility of changing intensities and thus the diffeomorphism does not apply to intensity displacement.
} 
demons [13], spatial deformation can be directly performed in the Lie group of diffeomorphisms. Thus, the registration results are diffeomorphic when the Lie algebra is applied during the optimization, avoiding the typical routine of adding constraints into the objective. Following this idea, we adopt the intrinsic updating rule:

$$
s \leftarrow s \circ \exp (u)
$$

for the evolution of the spatial deformation field. Here, $s$ is the overall spatial deformation and $u$ contains the first three components of the surface evolution (4.10): $u=\left[\frac{\partial X^{1}}{\partial t} \frac{\partial X^{2}}{\partial t} \frac{\partial X^{3}}{\partial t}\right]^{\prime}$. The intensity displacement, on the other hand, is directly accumulated as follows

$$
\Delta \leftarrow \Delta+\delta,
$$

where $\Delta$ is the overall intensity displacement, and $\delta$ is the last component of the surface evolution (4.10): $\delta=\frac{\partial X^{4}}{\partial t}$. After applying intensity displacement $\Delta$ on the target image $I_{f}$, given by

$$
I_{f}^{t+\Delta t}=I_{f}^{t}+\Delta
$$

where $t$ is the time variable, the intensity difference caused by topological changes will be eliminated. As a result, we refer to this step as intensity correction.

\subsubsection{Registration algorithm}

To summarize, we have the following registration algorithm:

\section{Algorithm (Image Registration using Embedded Maps):}

- Initialization:

1. set the image with normal topology as moving image $I_{m}$;

2. set the image with topological change as fixed image $I_{f}$;

3. construct $\beta$ using the binary label map of topological change, $L$ (4.13) (4.7);

4. set deformation field $s=I d$ and intensity displacement $\Delta=0$.

- iteration $t$ :

1. compute the update for surface evolution (4.10): $u=\left[\frac{\partial X^{1}}{\partial t} \frac{\partial X^{2}}{\partial t} \frac{\partial X^{3}}{\partial t}\right]^{T}$ and $\delta=\frac{\partial X^{4}}{\partial t}$;

2. apply fluid regularization on $u: u \leftarrow K_{\text {fluid }} * u$; 
3. update deformation field: $s \leftarrow s \circ \exp (u)$;

4. apply diffusion regularization on $s: s \leftarrow K_{\text {diff }} * s$;

5. update intensity displacement $\Delta \leftarrow \Delta+K_{\text {int }} * \delta$;

6. apply spatial deformation $I_{m}^{t+1}=s\left(I_{m}^{t}\right)$;

7. apply intensity dispalcement $I_{f}^{t+1}=I_{f}^{t}+\Delta$;

8. compute new total error: TotalError $=\Sigma\left|I_{m}^{t+1}-I_{f}^{t+1}\right|^{2}$;

9. if (TotalError $<$ threshold $\| \mathrm{t}=$ maxIteration) break;

Note that $u$ and $\delta$ are both normalized and multiplied by a chosen step size as in typical registration methods. Following the convention in diffeomorphic demons, the step size for spatial deformation is set to a constant of 2 pixels, and the step size of intensity displacement can be set to $\frac{\text { maximum image intensity }}{\text { number of iterations }}$.

\subsection{Implementation details}

The proposed algorithm is implemented using the $\mathrm{C}++$ image processing library Insight Toolkit (ITK) [11]. The implementation includes the following list of files:

- REMRegistration.cxx

- ExponentialDeformationFieldImageFilter.h

- ExponentialDeformationFieldImageFilter.txx

- GaussianSmoothingVectorFieldFilterByD.h

- GaussianSmoothingVectorFieldFilterByD.txx

- CMakeLists.txt

After collecting all the inputs, the source and target pass through an optional smoothing step, where smoothing will result in low SNR but lose of image information to a certain extent. This smoothing step is not suggested in most applications. Then the source image is histogram matched to the target image. 
Before entering the registration loop, several intermediate variables are initialized, including $u, \delta, s, \Delta$, warped moving image and $\beta$. Then, within each iteration, we first update $\phi$ function via calling the itkSquaredDifferenceImageFilter. The first and second order partial derivatives of $I, \beta$ and $\phi$ are computed using the itkGradientRecursiveGaussianImageFilter and the itkHessianRecursiveGaussianImageFilter. The surface variation is then computed using (4.10).

The fluid regularization and the diffusion regularization are implemented using Gaussian smoothing GaussianSmoothingVectorFieldFilterByD. Then, both $u$ and $\delta$ are normalized and multiplied by the chosen step sizes. To map $u$ into the Lie group of diffeomorphisms, we used the ExponentialDeformationFieldImageFilter, which is originally included in the diffeomorphic demons package and was edited for our application. The update of the deformation field given in (4.14) is implemented by spatially warping the deformation field $s$ by $u$ using itkWarpImageFilter and then incorporate $u$ using itkAddImageFilter. The intensity displacement is applied also using itkAddImageFilter.

After the update, image $L_{2}$ residual is re-computed for the current iteration and $\phi$ function is updated. This process repeats until the maximum iteration is reached.

The arguments and their common values of our deformable registration algorithm are listed as follows:

1. $\operatorname{argv}[1]$ source image (healthy subject/template)

2. $\operatorname{argv}[2]$ target image (image with topological change)

3. $\operatorname{argv}[3]$ lesion_probability map

- binary lesion segmentation smoothed using Gaussian kernel of variance 1 works for most cases.

4. $\operatorname{argv}[4]$ Boolen: smooth_input_image

- 0 not to smooth

- 1 smooth

5. $\operatorname{argv}[5]$ float: sigma for fluid-like regularization

- 0.8 when template used as source image

- 1.2 when healthy subject is used as source image. 
6. agrv[6] float: sigma for diffusion-like regularization

- 0.8 when template used as source image

- 1.2 when healthy subject is used as source image.

7. $\operatorname{argv}[7]$ float: step Size for spatial deformation

- 2 works for almost all cases, same as in diffeomorphic demons

8. $\operatorname{argv}[8]$ float: step Size for intensity displacement

- 0 for healthy to healthy registration

- 0.05 for template to subject

- 0.01 for subject to subject.

9. agrv[9] float: epsilon 0.02

10. agrv[10] int: number of iterations

- 30 for template to subject

- 100 for subject to subject.

11. $\operatorname{argv}[11]$ output prefix

In the following, we list a set of sample arguments used to generate the results in Sec. 4.3.

- Sample arguments for Sec. 4.4.1:

./REMrederive OASISelderlyTemplate.nrrd OASIS_img0027.nrrd Oasis_0027_lesionMap.nii 00.80 .820 .050 .0230 templateToOASIS0027Reg

- Sample arguments for Sec. 4.4.2:

./REMrederive Oasissubj_0116.nrrd ADNI_brain0023.nrrd ADNI_lesionMap_0023.nrrd 01.21 .220 .010 .02100 OASIS0116ToADNI0023Reg

- Sample arguments for Sec. 4.4.5:

./REMrederive Oasis_subj_0050.nrrd Oasis_subj_0034.nrrd allZero_lesionMap.nrrd 01.21 .2200 .02 100OASIS0050ToOASIS0034Reg

./REMrederive OASISelderlyTemplate.nrrd Oasis_subj_0034.nrrd allZero_lesionMap.nrrd 00.80 .8200 .0230 templateToOASIS0034Reg 


\subsection{Experiments and results}

In this section, the registration algorithm is tested using multiple sets of experiments. The resulting deformation fields are compared with those obtained using diffeomorphic demons [13], where topological changes are not specially handled. Additional results are given to demonstrate the impact of false alarm and mis-detection of lesion in the construction of $\beta$. Experiments are also conducted for cases that are currently modeled, e.g., both moving and fixed images contain topological changes, to show the advantages as well as the limitations of the algorithm.

\subsubsection{Register template to brain MRIs with lesions.}

In the first set of experiments, we register a brain template to a set of 20 brain MR images with lesions. The template was constructed for one of our early studies [55], which is obtained by aligning and averaging a set of $130 \mathrm{MR}$ images for healthy elderly subjects (age above 58) from the OASIS dataset [56]. The testing set of 20 brain MR images with lesion were also from the OASIS dataset, but none of them were used in the template construction.

The template is affine registered to the target images before applying our deformable registration algorithm using the Slicer3 [57] Fast Affine Registration Module. Compared to MR images of individual subjects, brain templates are very smooth. As a result, in the experiments, we use small Gaussian kernels for the smoothing of deformation fields: $\sigma_{\text {fluid }}=\sigma_{\text {diff }}=0.8^{2}$ for $K_{\text {fluid }}$ and $K_{\text {diff }}$. Lesions are segmented using the FreeSurfer tool [17] (segmented with the label white matter hypointensity lesion), and $\beta$ is then constructed with $\sigma_{\text {prob }}=1$ for $K_{\text {prob }}$ in (4.13); $\epsilon=0.01$ in (4.7). Other parameters are set as follows: $\sigma_{\text {prob }}=1$ for $K_{\text {prob }}$ in (4.13); $\epsilon=0.01$ in (4.7) and $\sigma_{i n t}=0.4$ for $K_{i n t}$. In all of the 20 registration experiments, the image residual stops dropping and begins oscillating ${ }^{3}$ within 15 -25 iterations, so we terminate all the registrations at the 30th iteration. Sample curves plotting the registration residual during the registration process are shown in Fig. 4.3(a), where the solid line plots image $L_{2}$ residual after the spatial deformation field of the current iteration is applied to the moving image, and the dashed line plots image $L_{2}$ residual after the intensity displacement is additionally applied. From these curves, we observe a smooth diffusion process both on

\footnotetext{
${ }^{2}$ All the parameter settings through this chapter are in pixel scale.

${ }^{3}$ Residual oscillation is a commonly seen phenomenon in gradient descent based searching. The reason mainly attributes to the over-shooting and back-and-forth searching when the current solution is close to the true local minimum.
} 

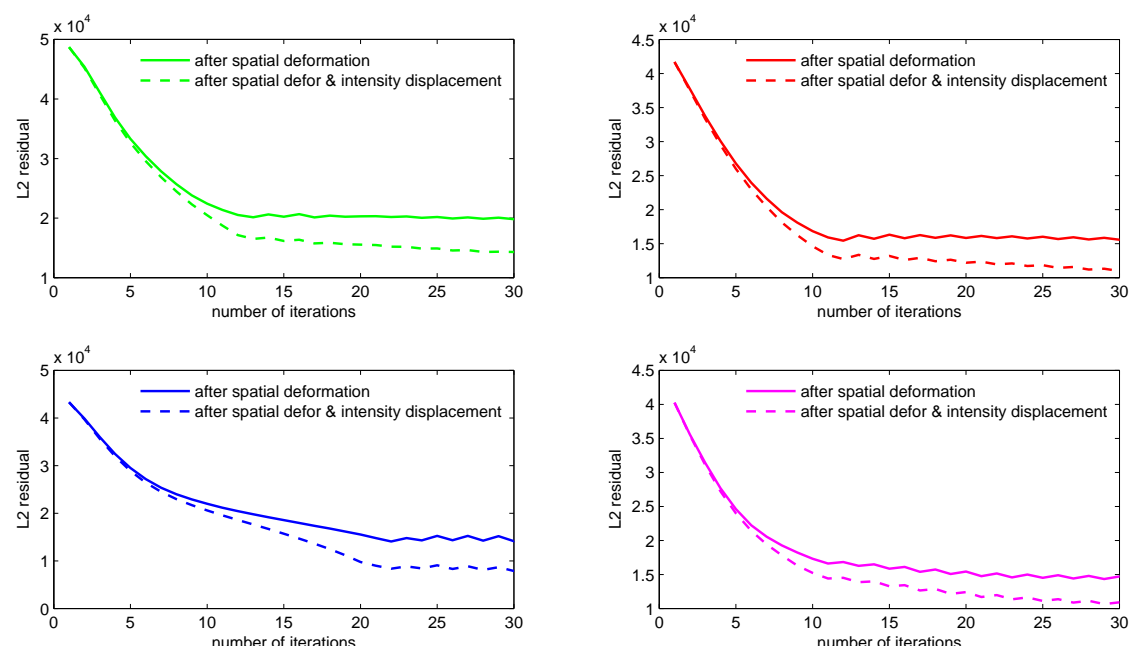

(a)

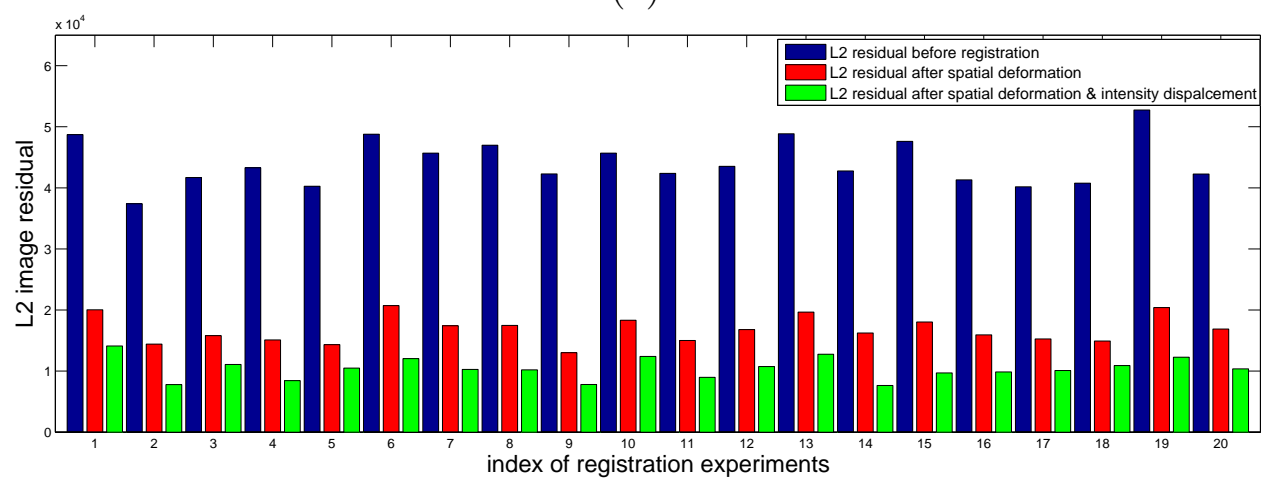

(b)

Figure 4.3: Registration Residual for the first set of registrations. (a) Sample curves plotting the registration residual during the registration process. The solid line plots the image $L_{2}$ residual after the spatial deformation field of the current iteration is applied on the moving image, and the solid line plots the image $L_{2}$ residual when the intensity displacement is also applied. (b) Image $L_{2}$ residuals before and after registration. The blue bars shows the $L_{2}$ distance between the source and target image before deformable registration. The red bars show the $L_{2}$ residual between spatially deformed moving image and the target images. The green bars show the $L_{2}$ residual after the intensity displacement is further applied to the target image. 
the evolution of spatial deformation and on that of the intensity displacement. The image $L_{2}$ residuals before and after registration for all the 20 experiments are shown in Fig. 4.3(b). The blue bar shows the $L_{2}$ residual between the moving and the target images before our deformable registration. The red bar shows the $L_{2}$ residual between the spatially-deformed moving image and the target image, where we can see a significant drop of residual values compared to the corresponding blue bar. We also observe a further drop of residual values on the green bars, which are the $L_{2}$ residuals after the intensity displacement is applied to the target image, in addition to spatial deformation. In other words, the green bar shows the registration residual after removing the contribution from the intensity difference caused by topological change.

Some samples of the registration results are given in Fig. 4.4 to Fig. 4.7. Fig. 4.4 to Fig. 4.7 each shows the result of one registration experiment, where the first row shows the checkerboard image of the template and the target image. The second row shows the checkerboard image of the target image and the registered template, i.e., after spatial deformation. The target images all contain lesions, which are segmented using the FreeSurfer tool [17] (segmented with the label white matter hypointensity lesion), and marked out using blue contours, as shown in the third rows. The fourth rows give the target images after intensity displacement is applied. The fifth and sixth rows in Fig. 4.4 to Fig. 4.7 plot the iso-contours of the moving image on top of the target image, before and after the spatial deformation is applied, respectively. These iso-contour plots help to better visualize the alignment of cortical gray matter (GM) and white matter (WM). The three columns in Fig. 4.4 to Fig. 4.7 show the Axial, Sagittal and Coronal views of each volume, respectively. For all the cases, we can see that the anatomical structures of the template are well aligned with those on the target images and the intensity within lesions in the target image is corrected to become that of healthy white matter.

Fig. 4.8 provides some samples of the resulting deformation vector fields using a glyph view $^{4}$. The template image (the left-most column) is registered to two subjects with severe lesions (the second column). After the registration, the template image is deformed to align with the subjects, as shown in the third column. In the last column, we show a zoomed view where the deformation fields are plotted as glyph fields on top of the target image. For the first subject (the upper row), we focus on the lesions around the first ventricle, and for the second subject (the lower row), we focus on the lesion around the lateral ventricle. From

\footnotetext{
${ }^{4}$ Note that the arrows in the glyph plot are only for illustration, and vector may not be length to scale, to improve visualization. This applies to all the following glyph plots.
} 

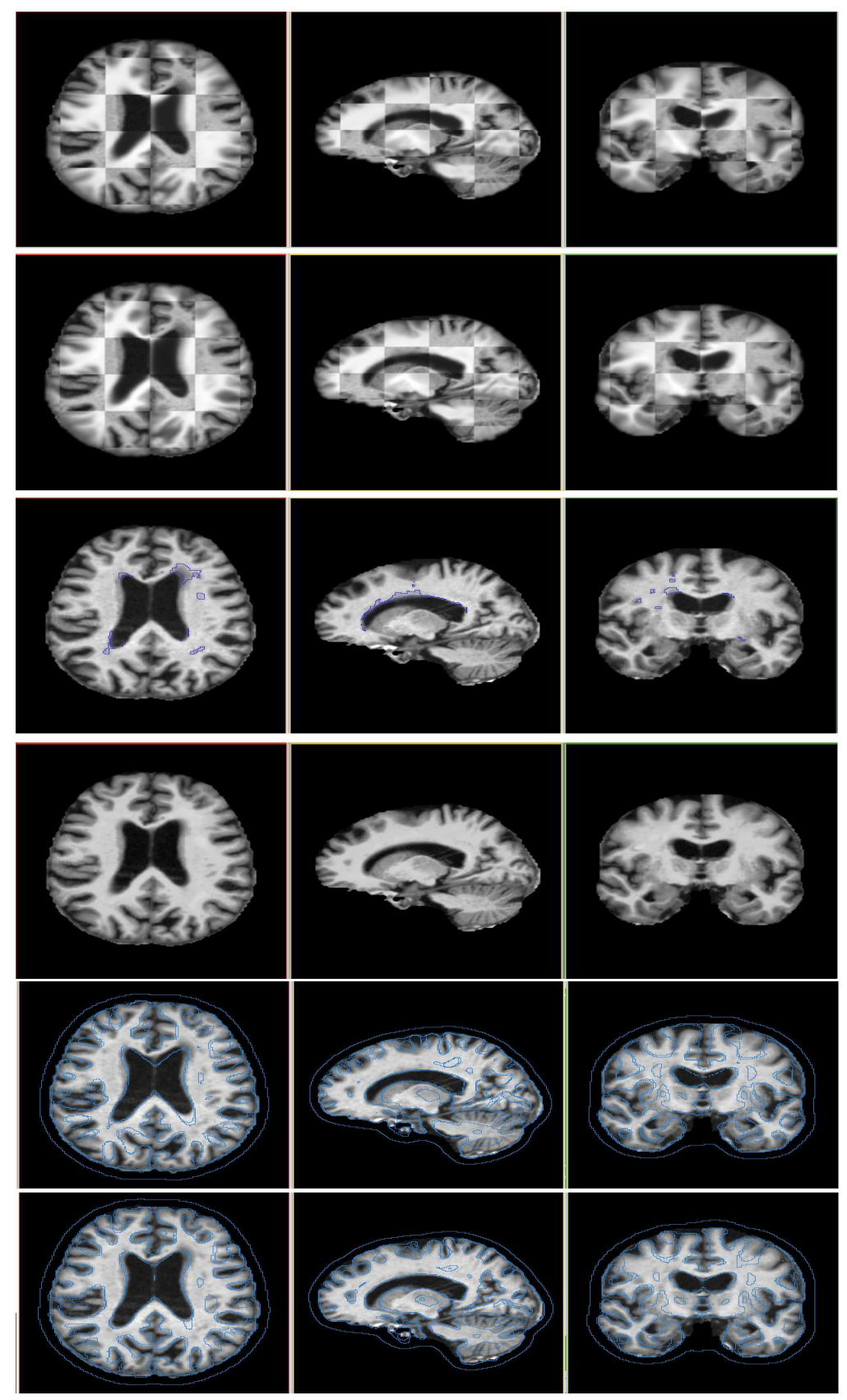

Figure 4.4: Sample of registration results 1: from a template to subjects with lesion from OASIS dataset. The first row shows the template image which is affinely aligned to the target image. The second row shows the target images that containing lesions, which are marked out with blue contours. The third row shows the spatially deformed template image, and the last row gives the target image after intensity displacement is applied. The fifth and sixth rows plot the iso-contours of the moving image on top of the target image, before and after the spatial deformation is applied, respectively. The columns show the Axial, Sagittal and Coronal views, from left to right, respectively. 

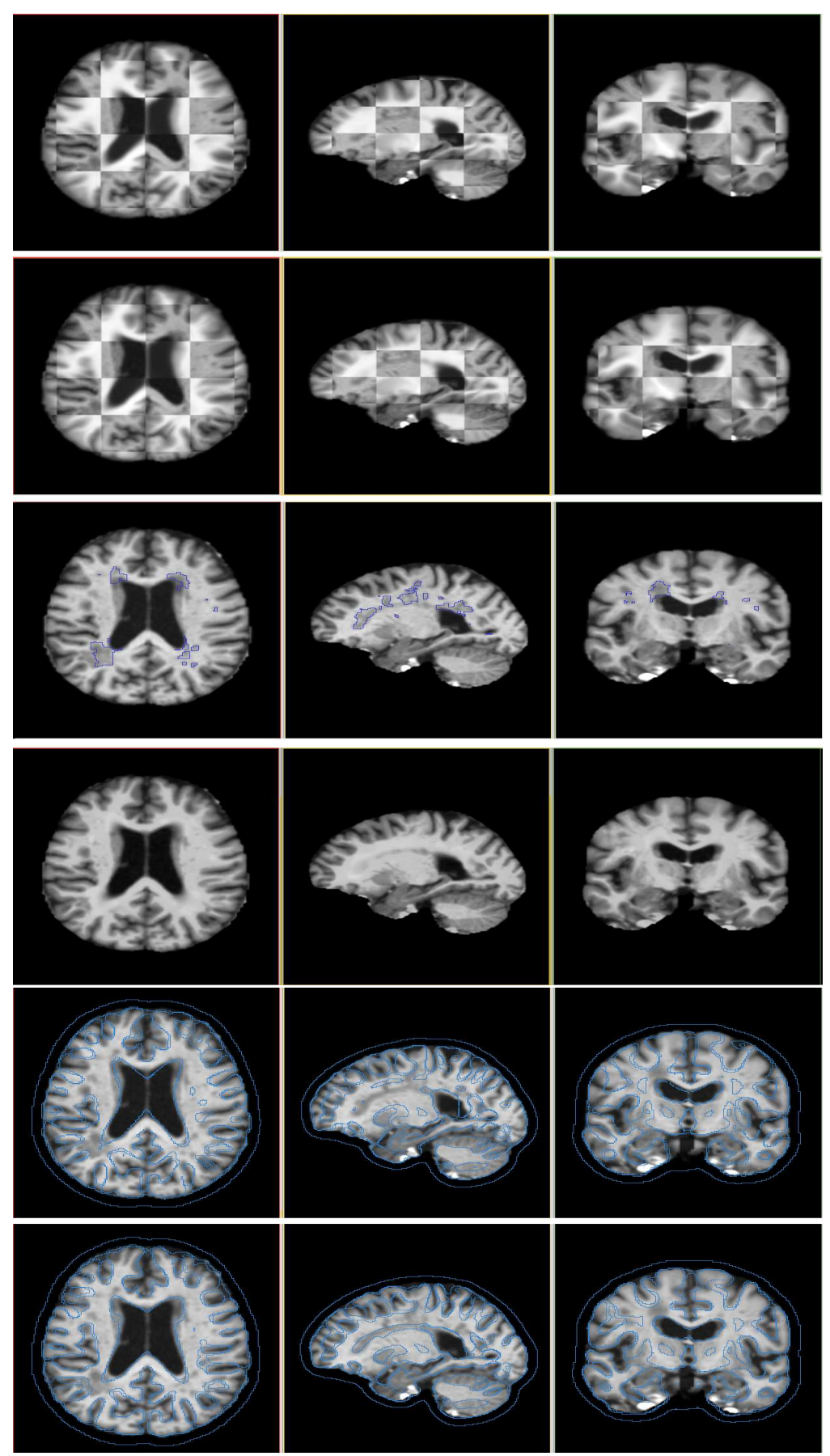

Figure 4.5: Sample of registration results 2. Subfigures are organized in the same way as in Fig. 4.4. 

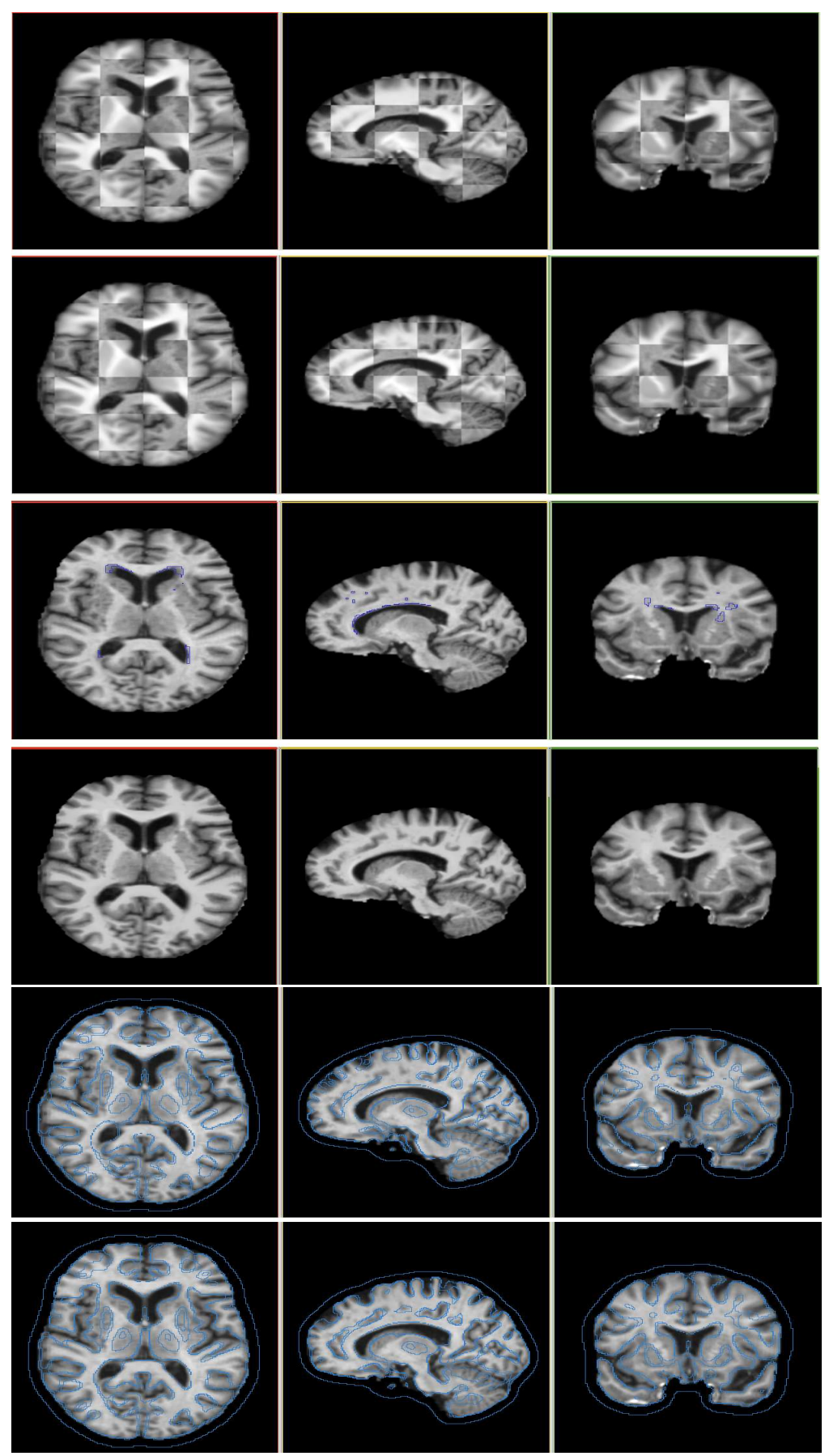

Figure 4.6: Sample of registration results 3. Subfigures are organized in the same way as in Fig. 4.4. 

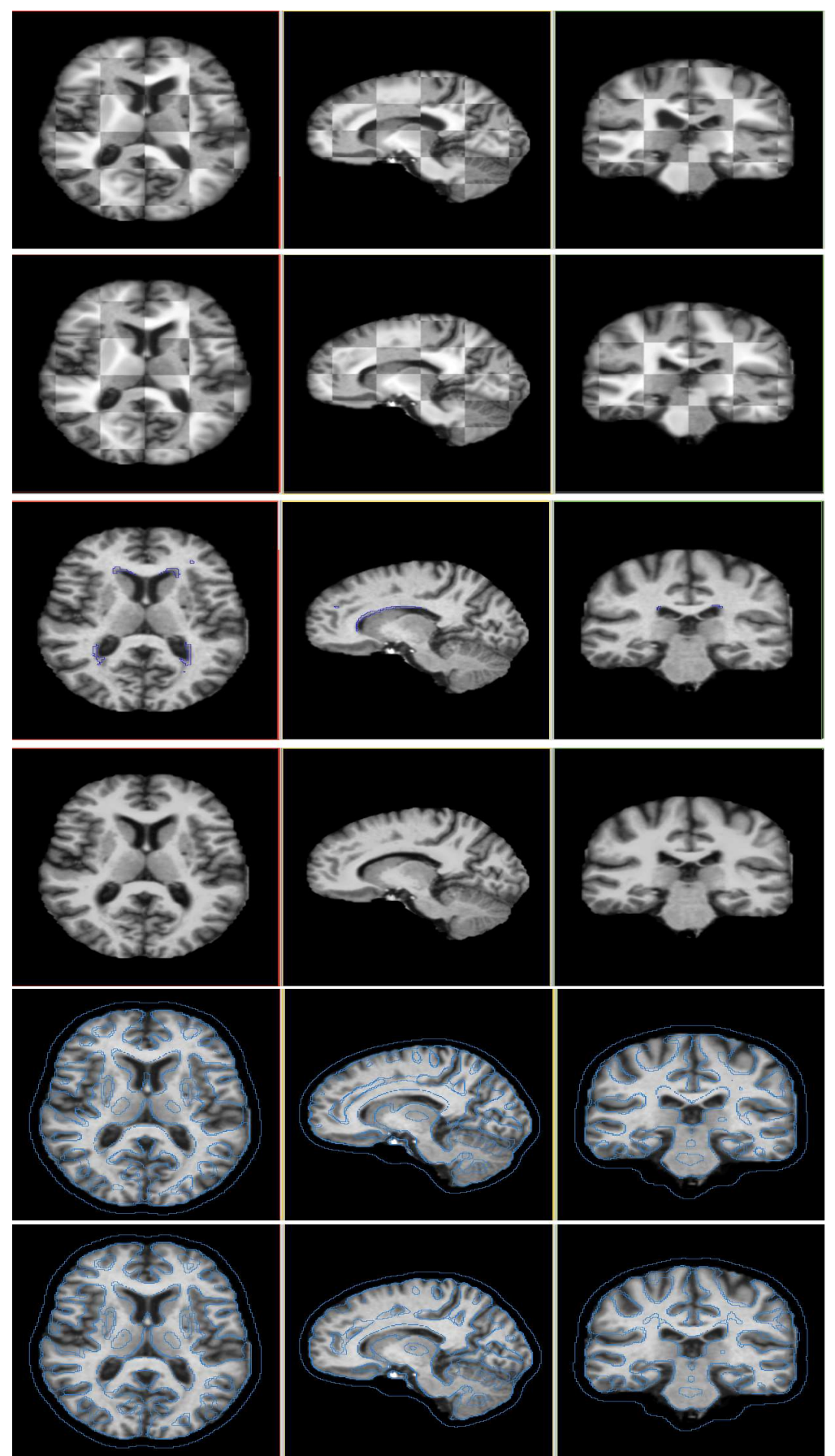

Figure 4.7: Sample of registration results 4. Subfigures are organized in the same way as in Fig. 4.4. 


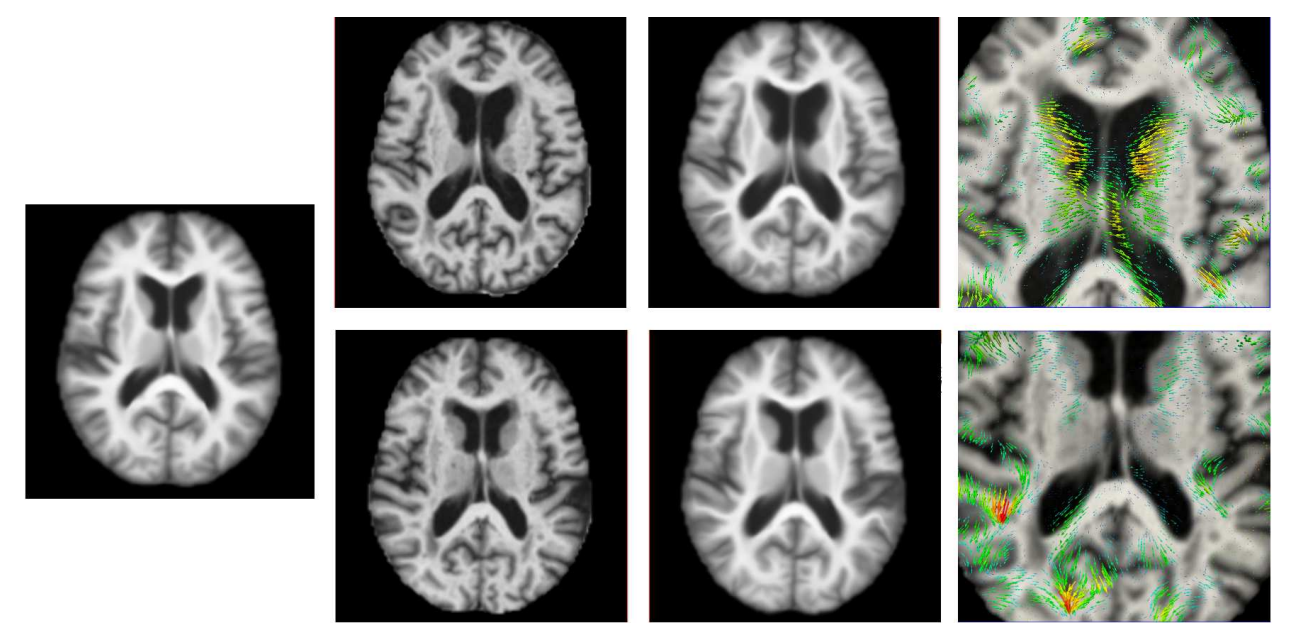

Figure 4.8: Samples of the resulting deformation vector fields from a template to subjects with lesion from OASIS dataset. Vector fields are shown using glyphs. Left-most column: the template image. Second column from left: two subjects with severe lesions. Third column from left: the spatially deformed template image after registration. Right-most column: zoomed view of deformation fields on top of the target image. For the first subject (upper row), zoomed region around the first ventricle. For the second subject (lower row), zoomed region around the lateral ventricle.

both cases, we found no false deformation around the lesion areas.

For comparison purposes, we also used the diffeomorphic demons algorithm to conduct these experiments. However, in all the experiments, diffeomorphic demons is not successful in the sense that the algorithm converges to the results that completely shrink the areas of white matter into a spike. This has been seen in our previous study when the smoothness of the moving and the target images differ significantly. In Fig. 4.9 and Fig. 4.10, we provide a comparison of the registration results when our algorithm is set to the same configuration as diffeomorphic demons: no smoothing on both the moving and the target images, $\sigma_{\text {fluid }}=$ $\sigma_{\text {diff }}=0.8$ for $K_{\text {fluid }}$ and $K_{\text {diff }}$. Three resolutions are used in the diffeomorphic demons registration, where 50, 50 and 200 iterations are performed in each resolution, respectively. In Fig. 4.9 and Fig. 4.10, the first column from left shows the template image, the second column shows the target image, the third column gives the result of our algorithm and the fourth column gives that of diffeomorphic demons. The first and third rows show the images in Axial and Coronal views, respectively. The second and fourth rows show the glyph plots of deformation fields in Axial and Coronal views, respectively. From these examples, we find that our algorithm converges to reasonable results, whereas diffeomorphic demons does not. 

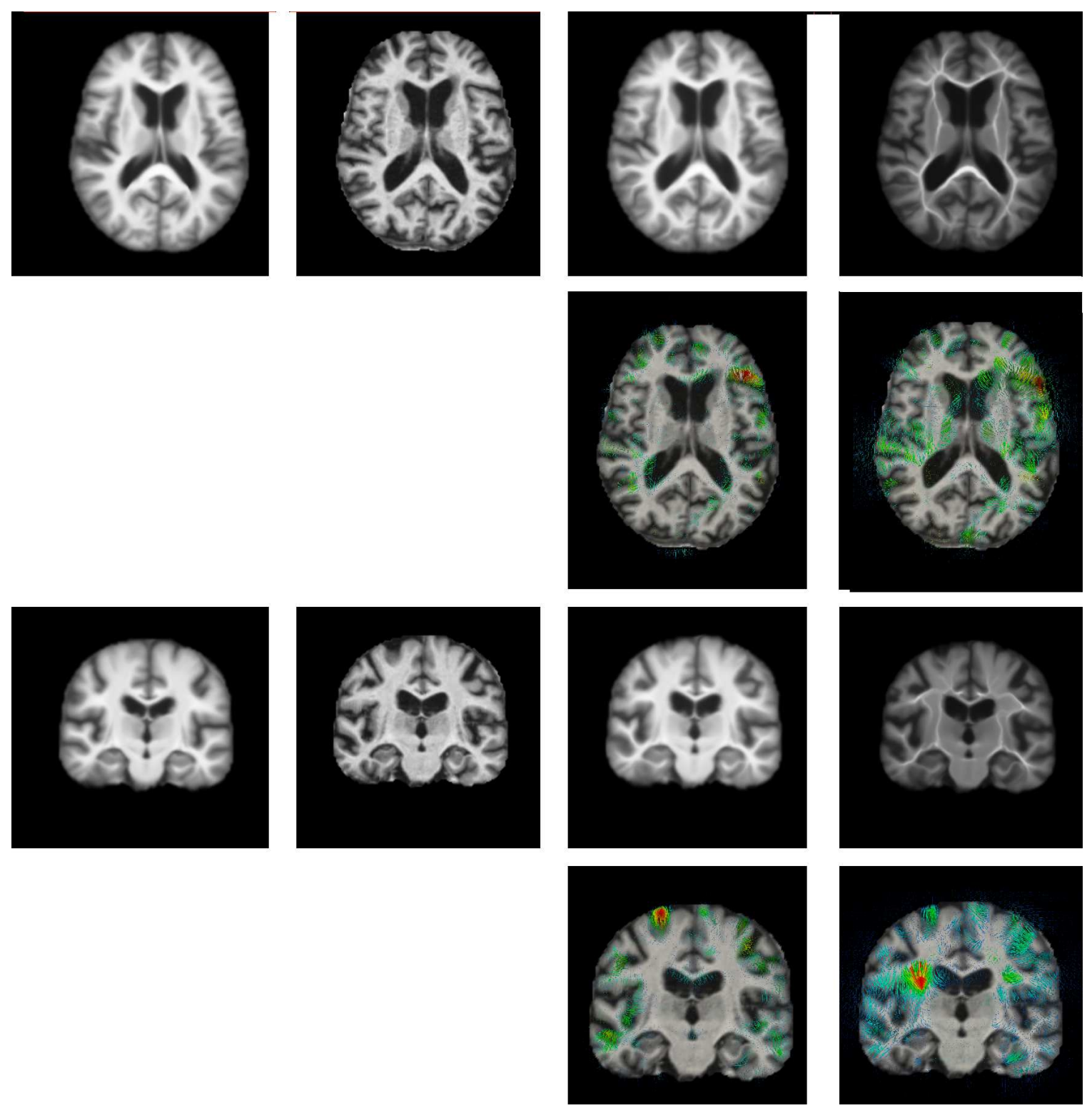

Figure 4.9: First example of comparing our registration result with that of diffeomorphic demons. The first column from left shows the template image, the second column shows the target image. The third column gives the result of our algorithm and the right-most column gives that from diffeomorphic demons. The first and third row shows the images in Axial and Coronal views, respectively. The second and fourth row shows the glyph plot of deformation fields in Axial and Coronal views, respectively. 

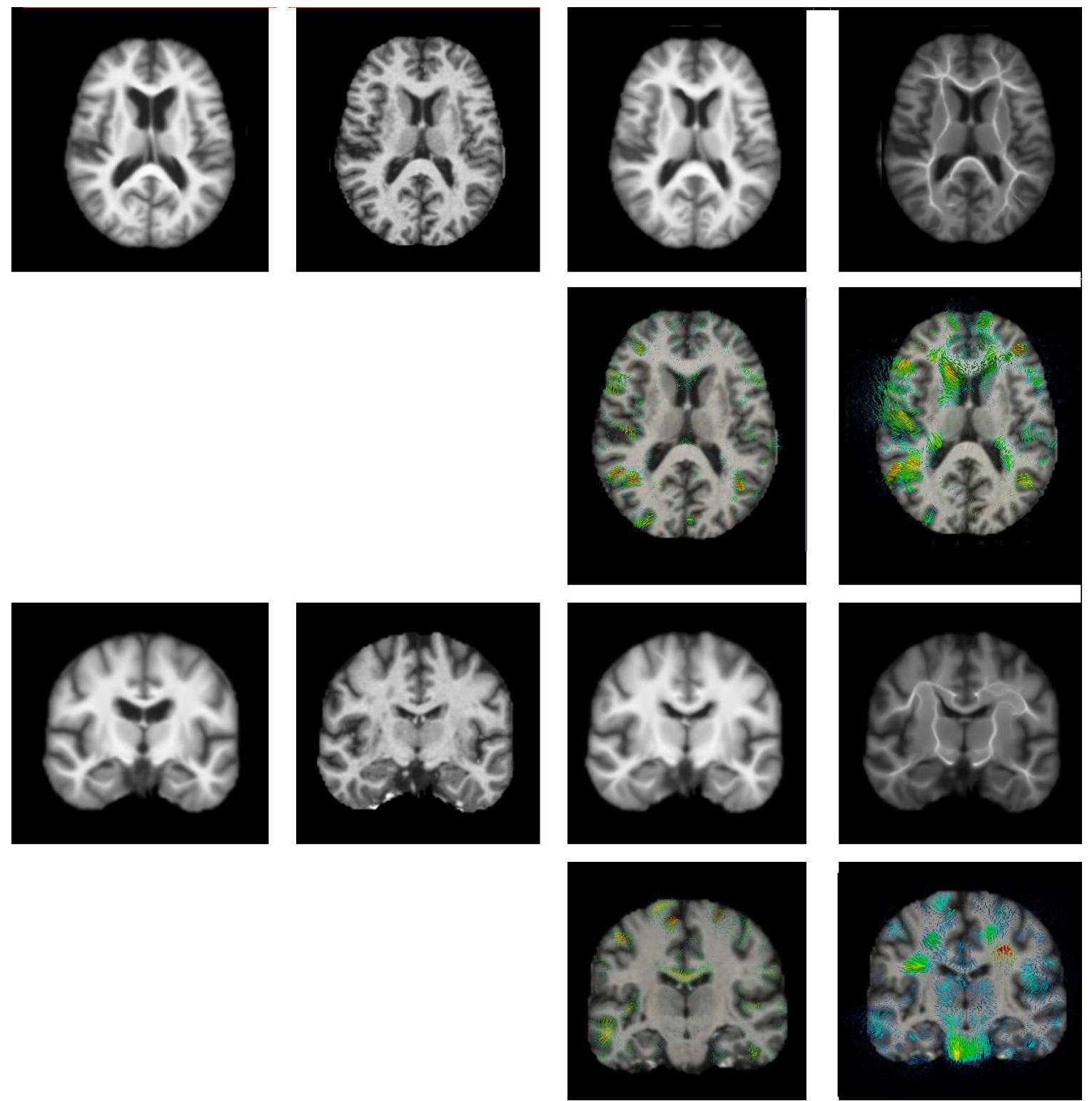

Figure 4.10: Another example of comparing our registration result vs. diffeomorphic demons. Subfigures are organized in the same way as in Fig. 4.9. 


\subsubsection{Register brain MRIs of healthy subject to that of Alzheimer's patients.}

In this set of experiments, we register the brain MR image of an age 77 healthy female from OASIS dataset to a set of 20 brain MR images with lesions in the Alzheimer's disease Neuroimaging Initiative (ADNI) dataset ${ }^{5}$. The images from the ADNI dataset are skullstripped using the FSL (BET) tool [58].

In these experiments, because we are conducting subject-to-subject registration, the moving image is noisier and sharper compared to the template image used in Sec. 4.4.1.A. As a result, we use larger Gaussian kernels for the smoothing of deformation fields, compared with those used in the previous set of experiments. Specifically, we use $\sigma_{\text {fluid }}=\sigma_{\text {diff }}=0.8$ for $K_{\text {fluid }}$ and $K_{\text {diff }}$, respectively. Other parameters are: $\sigma_{\text {prob }}=1$ for $K_{\text {prob }}$ in (4.13); $\epsilon=0.02$ in (4.7) and $\sigma_{i n t}=0.4$ for $K_{i n t}$. In the registration experiments, the moving image was first affinely registered to the target images, again using the Slicer3 [57] Fast Affine Registration Module. Then, when applying our deformable registration algorithm, the image residual stopped dropping and started oscillating after 50-80 iterations, so we terminate the registration for all experiments at the 100th iteration. Sample curves plotting the registration residual during the registration process for one of the experiments are shown in Fig. 4.11(b), with the same configuration as that used in Fig. 4.11(a). The image $L_{2}$ residuals before and after registration for all the 20 experiments are shown in Fig. 4.11(b), in the same fashion as used in Fig. 4.3(b). Samples of registration results are shown in Fig. 4.12 - Fig. 4.15. The subfigures are organized in the same fashion as in Fig. 4.4. We can see that in all the cases, the moving images are spatially deformed and well aligned with the target images, and the lesions in the target images are corrected after the intensity displacement is applied. As an alternative visualization, in Fig. 4.16 and Fig. 4.17, we provide the difference images between the moving and target images, before and after registration. The first rows show

\footnotetext{
${ }^{5}$ Data used in this chapter are selected from the Alzheimer's disease Neuroimaging Initiative (ADNI) database (http://www. loni.ucla.edu/ADNI). The ADNI was launched in 2003 by the National Institute on Aging (NIA), the National Institute of Biomedical Imaging and Bioengineering (NIBIB), the Food and Drug Administration (FDA), private pharmaceutical companies and non-profit organizations, as a $\$ 60$ million, 5-year public-private partnership. The primary goal of ADNI has been to test whether serial magnetic resonance imaging (MRI), positron emission tomography (PET), other biological markers, and clinical and neuropsychological assessment can be combined to measure the progression of mild cognitive impairment (MCI) and early Alzheimer's disease (AD). Determination of sensitive and specific markers of very early AD progression is intended to aid researchers and clinicians to develop new treatments and monitor their effectiveness, as well as lessen the time and cost of clinical trials.
} 
the difference images between the moving and target images before registration. The second rows give the difference images between the deformed moving image and the target image. The third rows provide the difference images between the deformed moving image and the lesion-corrected target image. We observe from these results, that the image differences caused by structural shape variation are removed after spatial deformation, and the image differences caused by lesion are further removed after applying intensity correction.

For comparison purposes, we again used diffeomorphic demons algorithm [13] to perform the same set of registration experiments. Three resolutions are used in the diffeomorphic demons registration, where 50, 50 and 200 iterations are performed in each resolution, respectively ${ }^{6}$. The variances used in the smoothing Gaussian kernel for fluid and diffusion regularization are both set to 1.2, the same as in our algorithm. In Fig. 4.11(b), we also use magenta bars to show the registration residual after applying diffeomorphic demons registration. We can see that in all cases, the residuals after applying diffeomorphic demons have values in between the residuals before and after intensity displacements (the red and green bars, respectively). It is intuitive to understand that, in diffeomorphic demons, part of the spatial deformation is contributed to local deformation that attempts to minimize the error caused by intensity difference of lesions. This will in turn cause false deformation.

Now we demonstrate the efficacy of our algorithm in eliminating false deformation. Fig. 4.18 (a) and (b) show the registration results using our proposed algorithm and diffeomorphic demons. In both (a) and (b), the sub-figures in the upper row, from left to right, show an axial view of the moving image, the target image, the registered moving image (after spatial deformation) using our algorithm, and the registered moving image (after spatial deformation) using diffeomorphic demons. In both cases, we find that the diffeomorphic demons algorithm tries to deform the lateral ventricle into the white matter areas with lesions, which leads to an incorrect registration result. In contrast, these errors are not seen in our registration results. Furthermore, in both (a) and (b), the left most sub-figure in the lower row shows a zoomed region in order to visualize the deformation field. The second and third sub-figures in both lower rows of (a) and (b) give the glyph view of the deformation field obtained using our method and diffeomorphic demons, respectively. For diffeomorphic demons, we observed a dense deformation energy concentration (abnormally long vectors) around the lesion-affected areas in the resulting deformation field, which is obviously false deformation. On the other hand, such false deformation is successfully removed in the

\footnotetext{
${ }^{6}$ Such multiple resolutions scheme can also be applied to our algorithm with helps to prevent convergence to local minimums. However, this scheme is currently not yet implemented.
} 
results obtained using our algorithm. We need to emphasize that, despite the advantages of our algorithm shown by these results, our algorithm does take lesion segmentation as an additional input which is important prior knowledge.

\subsubsection{Impact of false alarm and mis-detection of lesion.}

To eliminate false deformation, our registration method relies on an a-priori lesion estimation. However, the lesion segmentation obtained from automatic segmentation tools may contain errors, either poor segmentation or false alarm/min-detection. Here, we provide some examples to demonstrate the impact of these errors on our algorithm.

As explained in Sec. 4.2.2, $\beta$ is a function of a smoothed lesion segmentation. Fig. 4.19 shows such a case in our second set of experiments (Sec. 4.4.2). From Fig. 4.19 (a), we notice that FreeSurfer underestimates the lesion-affected areas (inside the blue contours), especially the lesion region to the left of the ventricle. Fig. 4.19 (b) shows the smoothing effect during the construction of the $p(x, y, z)$ function. All the lesions in the target image are successfully removed after intensity displacement, as shown in Fig. 4.19 (c). Finally, in Fig. 4.19 (d), we can see that the deformation field is not affected by the presence of a lesion, i.e., no false deformation is observed.

However, if a lesion-affected area is completely mis-detected, the registration performance will be affected. An example is shown in Fig. 4.20(a), which gives the Coronal view of a moving image, a target image, and a registered moving image after applying the spatial deformation, from left to right, respectively. We find that the skull stripping of the target image obtained from FSL (BET) is not perfect, where some extracranial tissue was kept as part of the brain. This tissue is not part of the brain, and does not have a matching anatomy structure in the moving image. Thus, it can be regarded as a topological change. In fact, such errors are commonly seen in large scale studies. Fig. 4.20(b) shows the deformation field around this area, where we observe some false deformation. Ideally, if this area was modeled in $\beta$, it would be corrected by intensity displacement and should not cause any spatial deformation.

Underestimation or even mis-detection is common in automatic lesion segmentation algorithms, due to the minor intensity differences of some lesions. However, it is relatively uncommon to see false alarms during lesion detection, since it is expected that there always exists some intensity difference. Thus, we do not have results to show the impact of false 

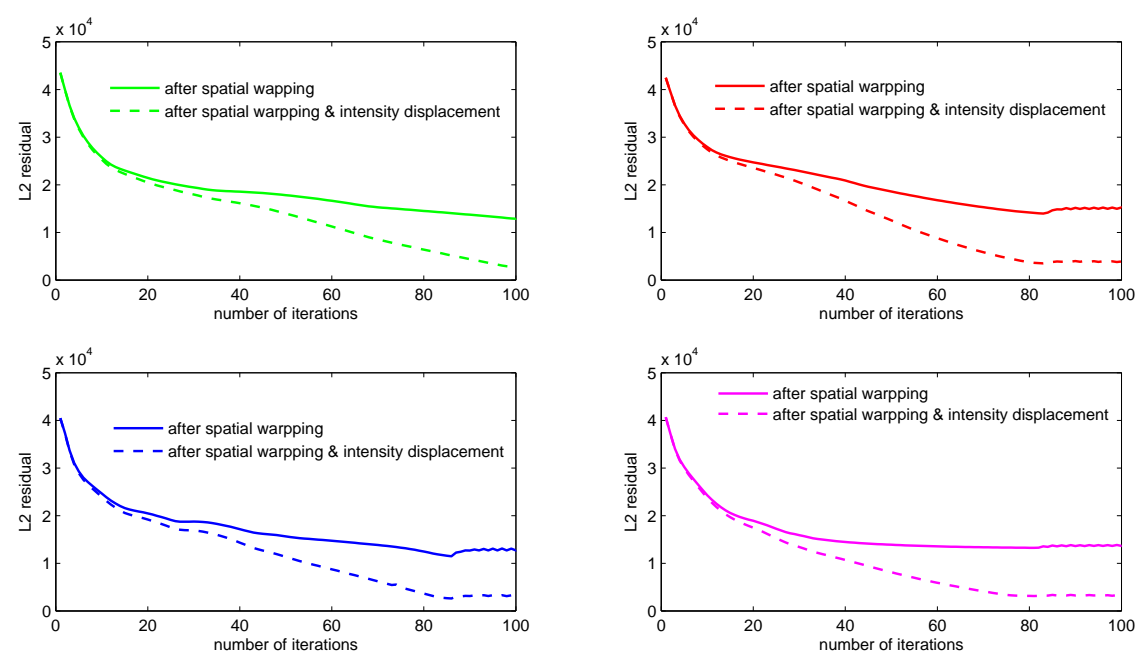

(a)

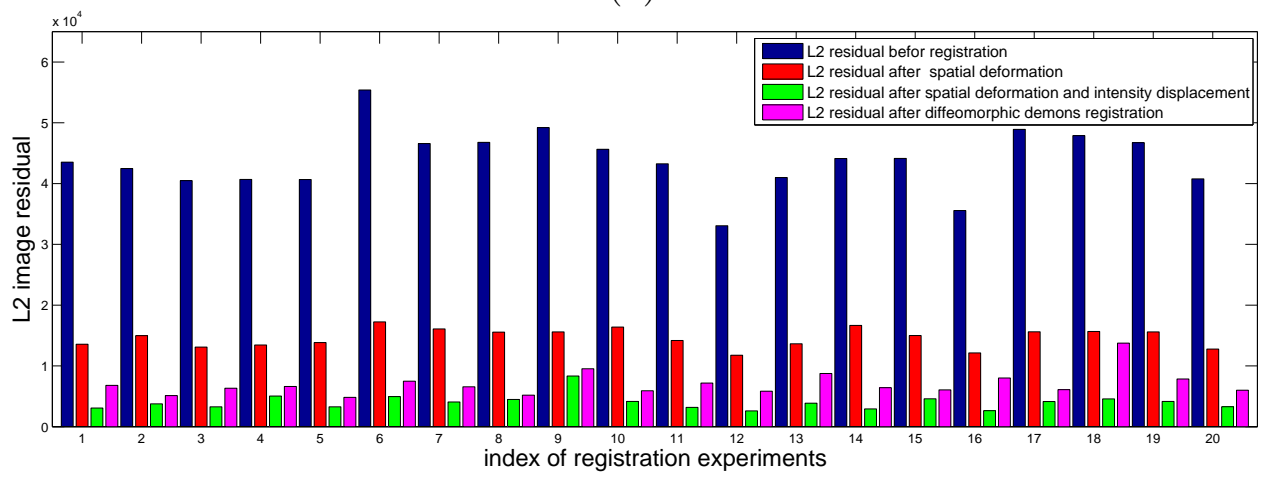

(b)

Figure 4.11: Registration residual for the second set of registration. (a): Sample curves plotting the registration residual during the registration process. The solid line plots the image $L_{2}$ residual after the spatial deformation field of current iteration is applied on the moving image, and the solid line plots the image $L_{2}$ residual when the intensity displacement is also applied. (b): Image $L_{2}$ residuals before and after registration. The blue bars shows the $L_{2}$ distance between the source and target image before deformable registration. The red bars show the $L_{2}$ residual between spatially deformed moving image and the target images. The green bars show the $L_{2}$ residual after the intensity displacement is further applied to the target image. In addition, the magenta bars in this subfigure show the $L_{2}$ residual after apply diffeomorphic demons registration. 


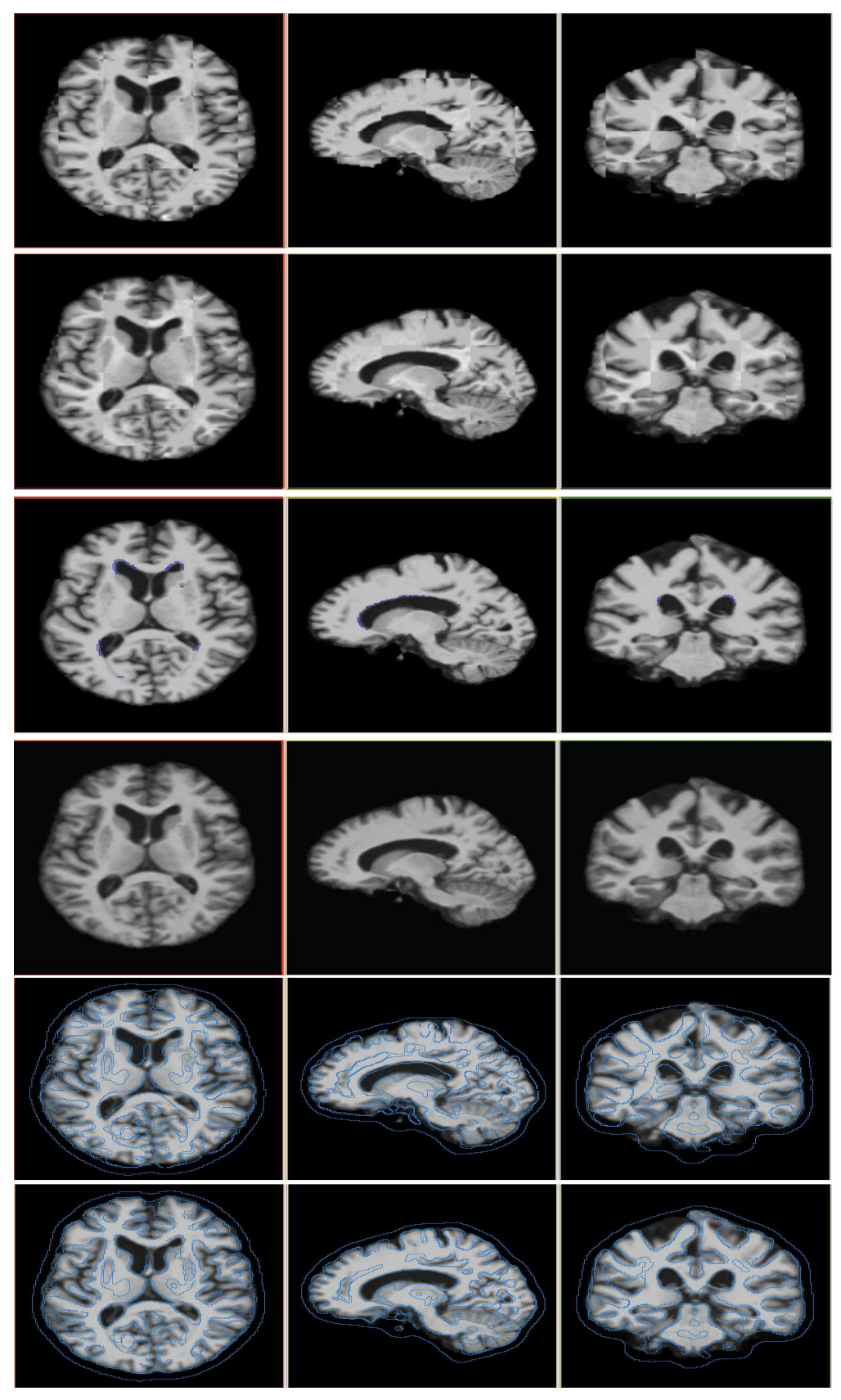

Figure 4.12: Sample of registration results 1: from a template to subjects with lesion from ADNI dataset. The first row shows the template image which is affinely aligned to the target image. The second row shows the target images that contain lesions, which are highlighted with blue contours. The third row shows the spatially deformed template image, and the last row gives the target image after intensity displacement is applied. The fifth and sixth rows plot the iso-contours of the moving image on top of the target image, before and after the spatial deformation is applied, respectively. The columns show the Axial, Sagittal and Coronal views, from left to right, respectively. 

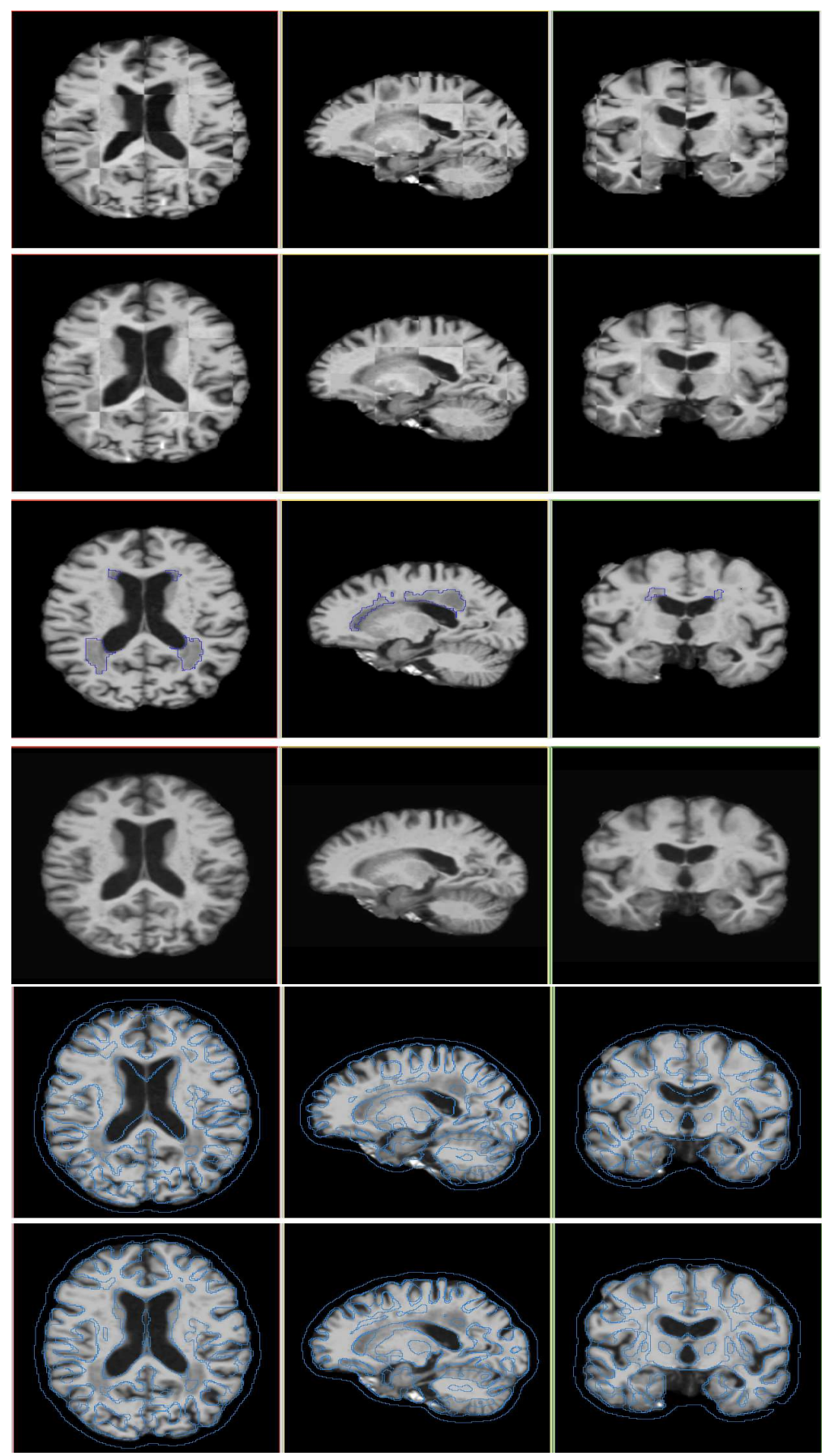

Figure 4.13: Sample of registration results 2. Subfigures are organized in the same way as in Fig. 4.12. 

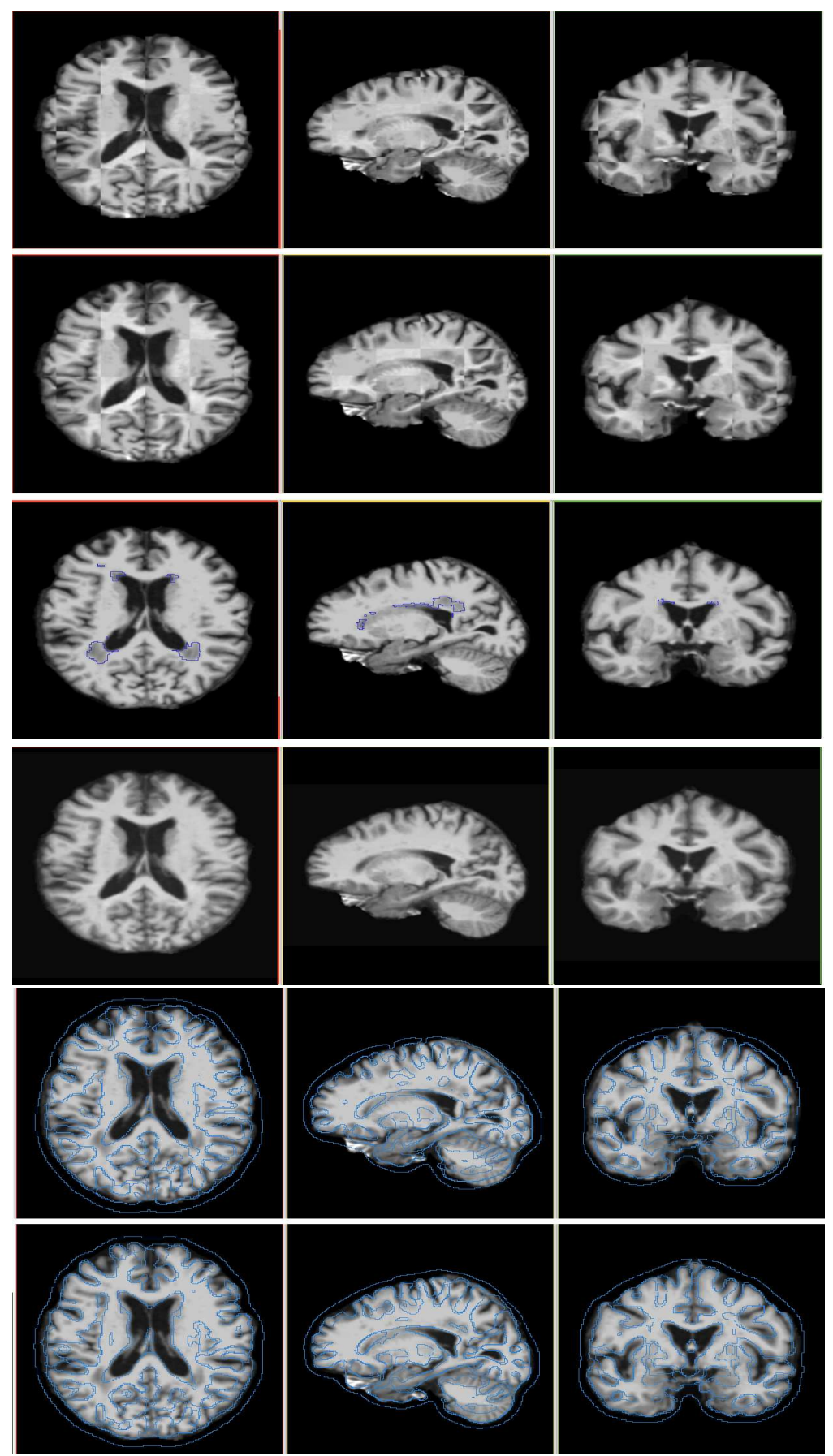

Figure 4.14: Sample of registration results 3. Subfigures are organized in the same way as in Fig. 4.12. 

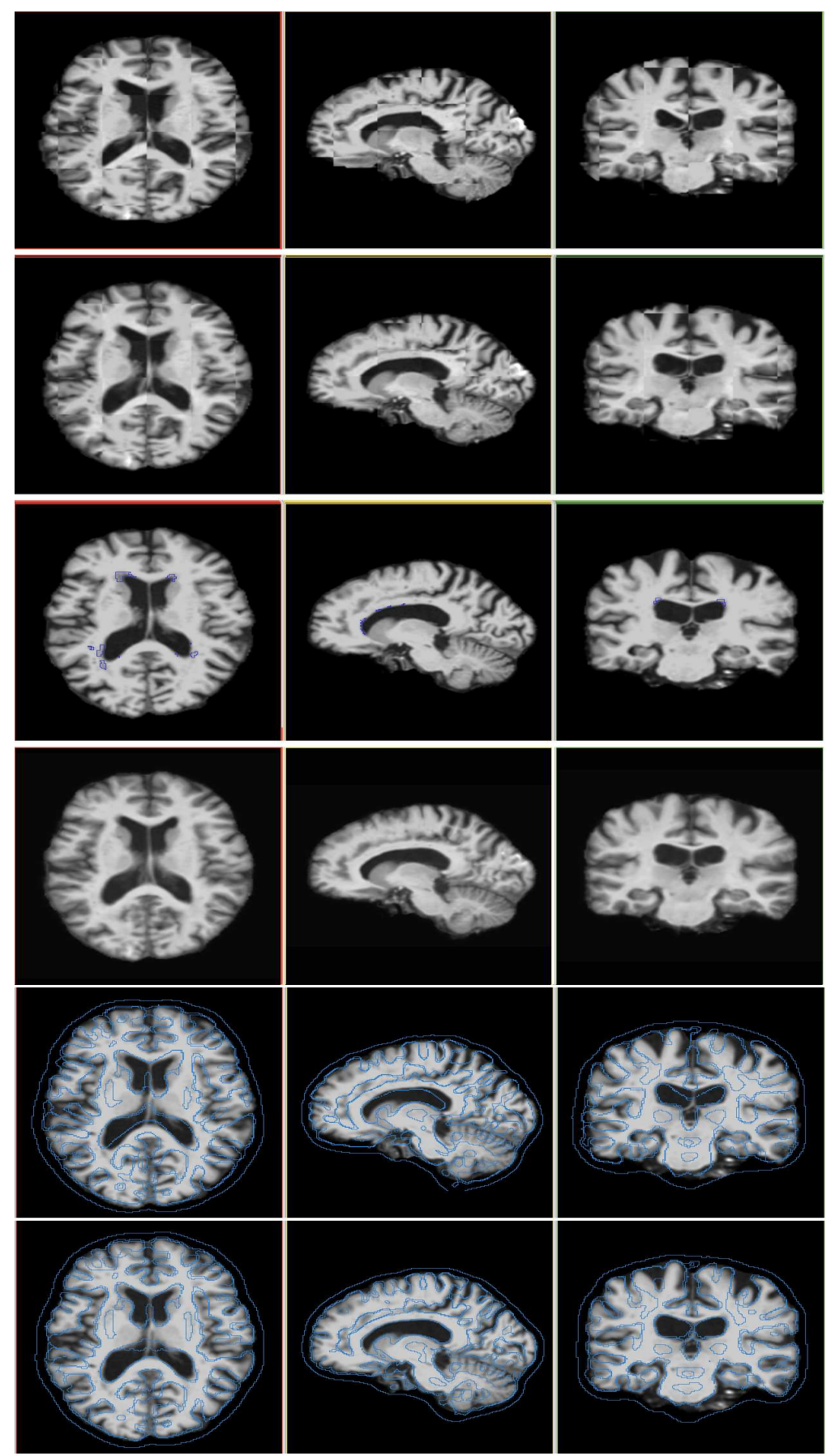

Figure 4.15: Sample of registration results 4. Subfigures are organized in the same way as in Fig. 4.12. 

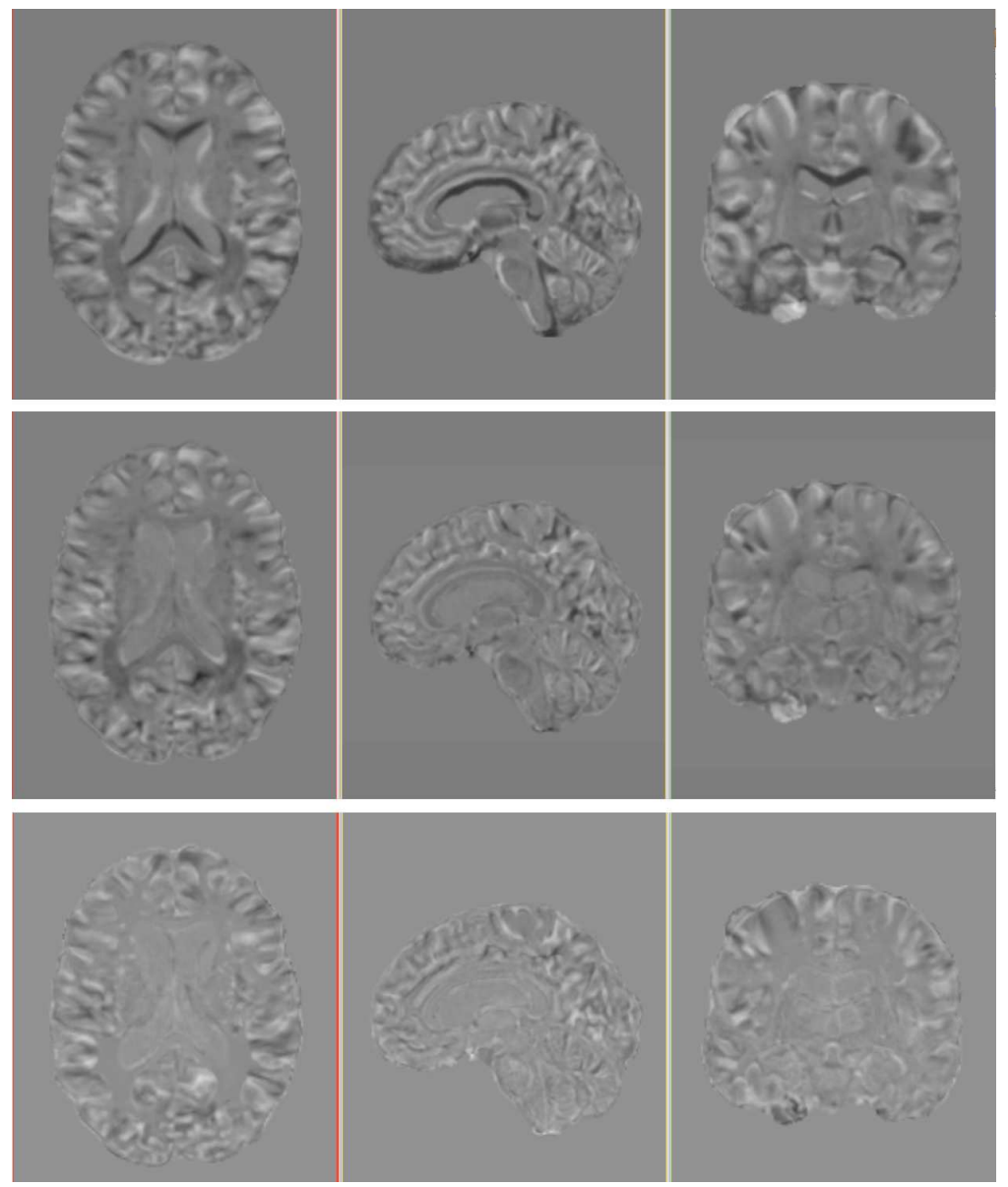

Figure 4.16: Sample of difference images 1. The first row shows the difference images between the moving and target images before registration. The second row gives the difference images between the deformed moving image and the target image. The third row provides the difference images between the deformed moving image and the lesion-corrected target image. 

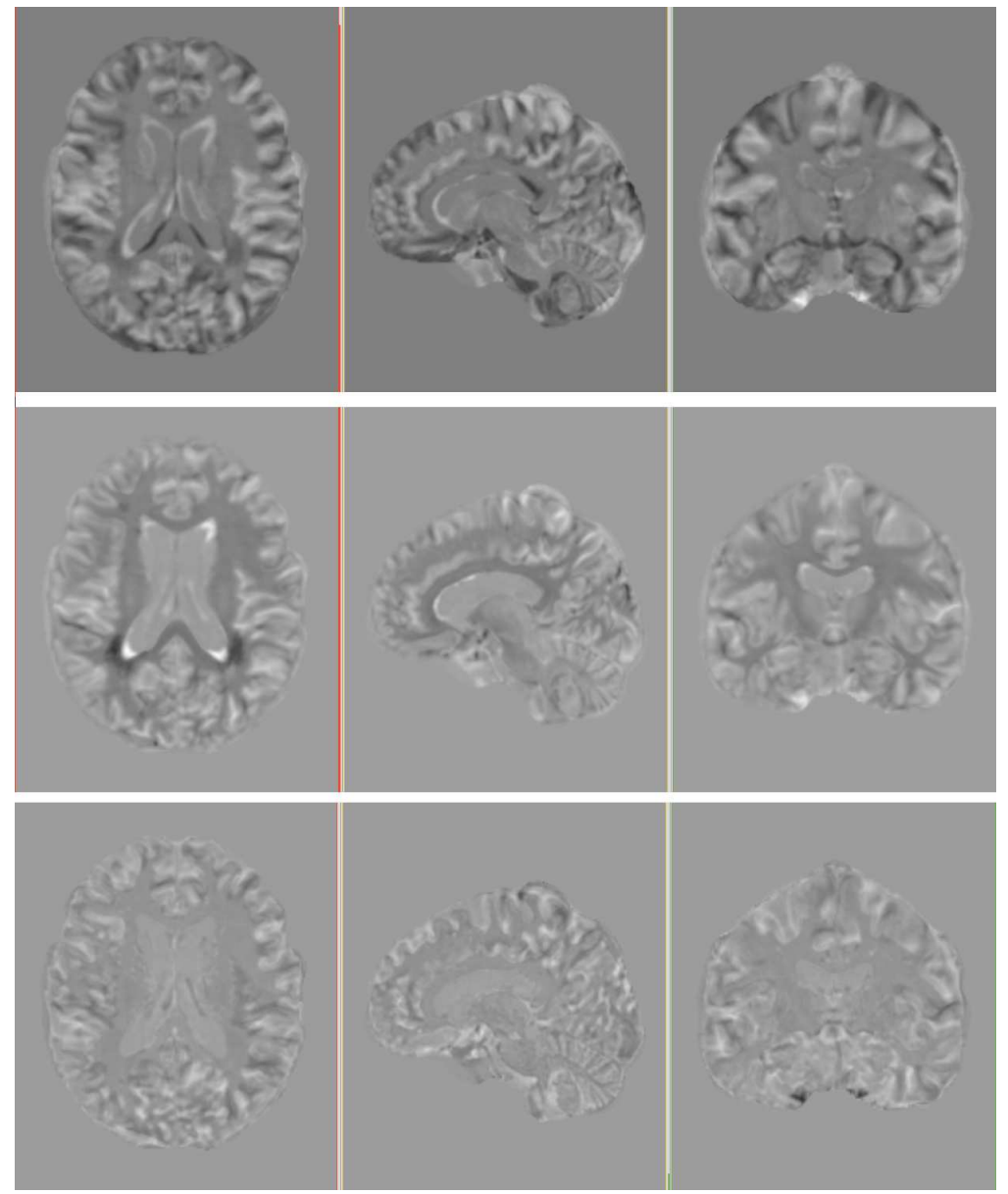

Figure 4.17: Sample of difference images 2. Subfigures are organized in the same way as in Fig. 4.16. 

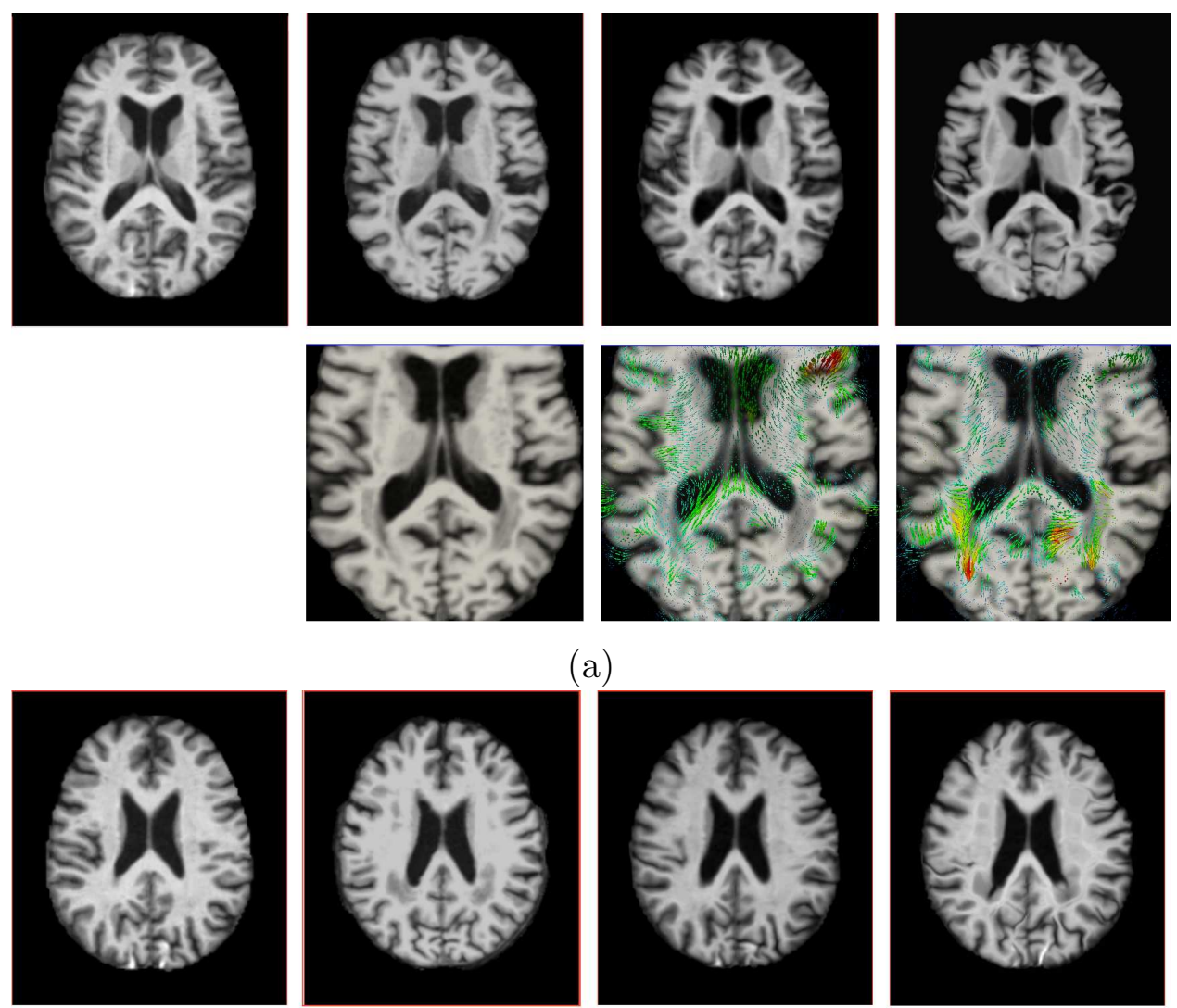

(a)
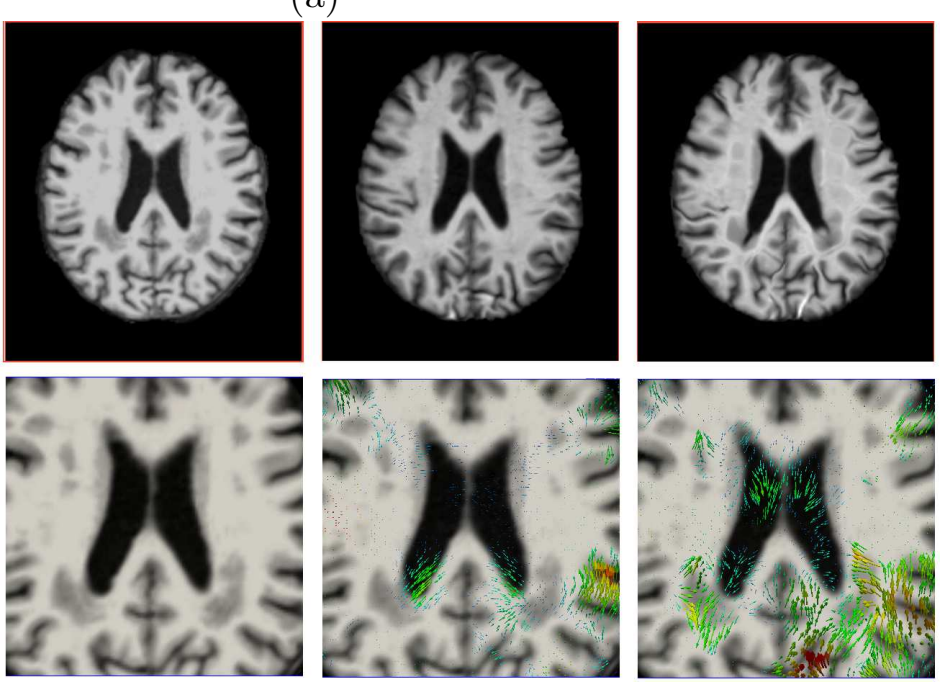

(b)

Figure 4.18: Impact of imprecise segmentation of lesion. In both (a) and (b), the subfigures in the upper row, from left to right, show an axial view of the moving image, the target image, the registered moving image (after spatial deformation) using our algorithm, and the registered moving image (after spatial deformation) using diffeomorphic demons. Also, in both (a) and (b), the left most subfigure in the lower row indicate a region that we zoom in to visualize the deformation field. The second and third subfigures from the left gives the glyph view of the deformation field obtained using our method and diffeomorphic demons, respectively. 


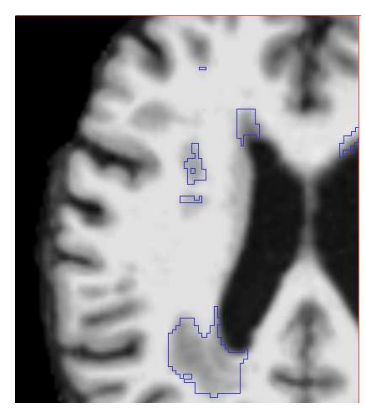

(a)

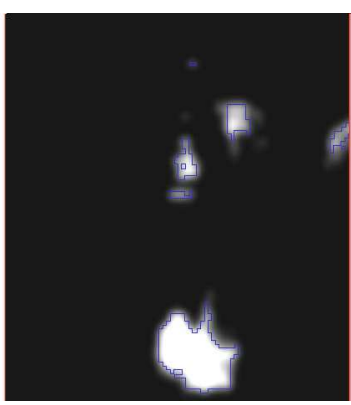

(b)

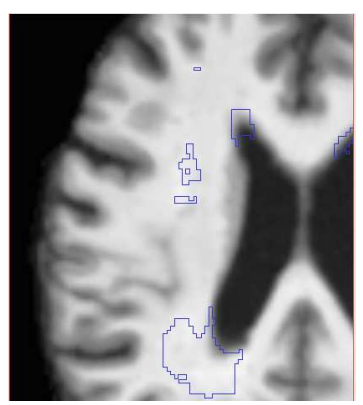

(c)

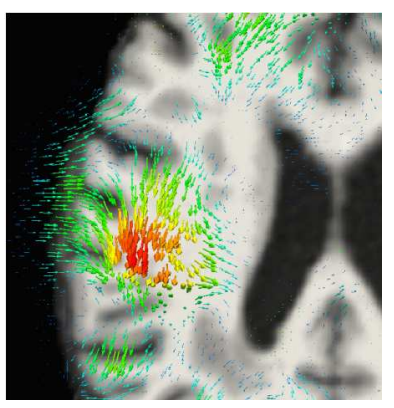

(d)

Figure 4.19: Sample of registration with imprecise segmentation. (a): lesion segmentation obtained using FreeSurfer, lesion areas are highlighted using blue contours, with part of lesions underestimated. (b): the constructed $\beta$ function. (c): result after intensity displacement applied on the target image. (d): the deformation field.

alarm of lesion detection in the two sets of experiments here. However, even with false alarm, we still expect our registration algorithm to perform normally. Imagine that if the intensities of certain region on the moving and the target images are very close, $\phi$ will have small values for these regions and no spatial deformation or intensity displacement will be exerted, even though $p(x, y, z)$ has large values.

Finally, we point out that the current version of our algorithm only deals with lesions in the target image and a normal anatomy of the moving image is expected. Fig. 4.21 shows the registration results when both the moving image (Fig. 4.21 (a)) and the target image (Fig. 4.21 (b)) have lesions (from the ADNI dataset). Only the lesion segmentation for the target image is modeled in $\beta$. We can observe that around the left side of lateral ventricle, the deformed moving image (Fig. 4.21 (c)) is not well aligned with the target image after registration, where false deformation can be observed, as shown in Fig. 4.21 (d).

\subsubsection{Example of registering a template to a brain image with a tumor.}

In this experiment, we register a brain MR image template, as shown in the first row of Fig. 4.22, to a target image that carries a brain tumor of considerable size, as shown in the second row of Fig. 4.22. The template image was constructed using 233 healthy young subjects (mean age 34.58) from OASIS dataset in our previous study [55]. The brain image with tumor was taken for a patient with a meningioma and is available in the testing data 

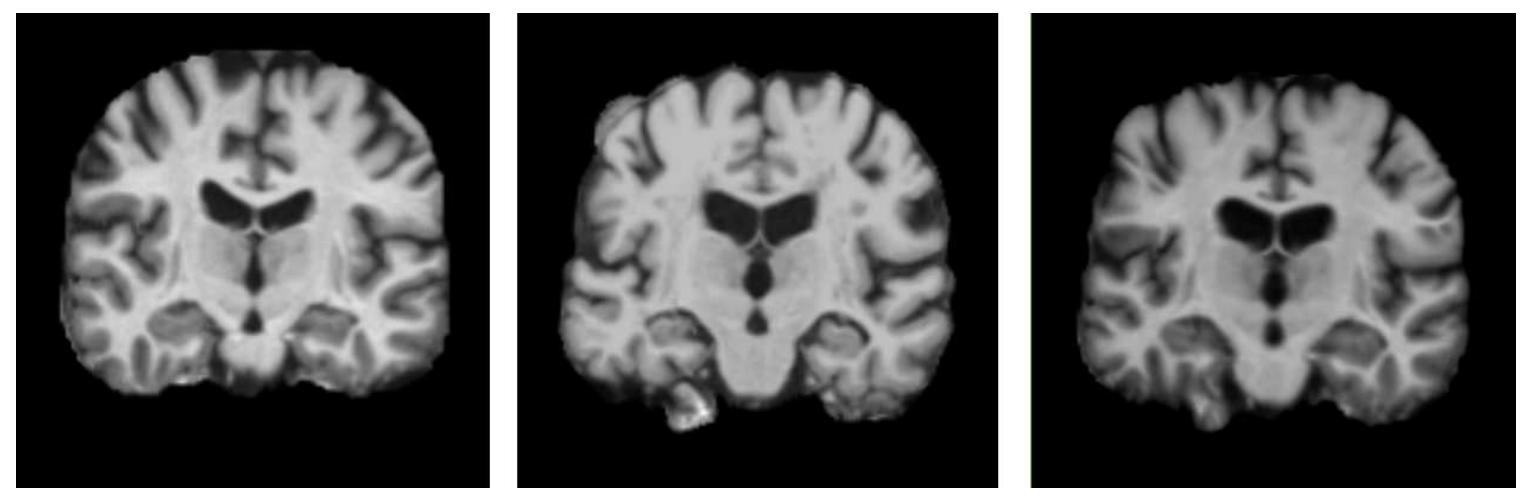

(a)

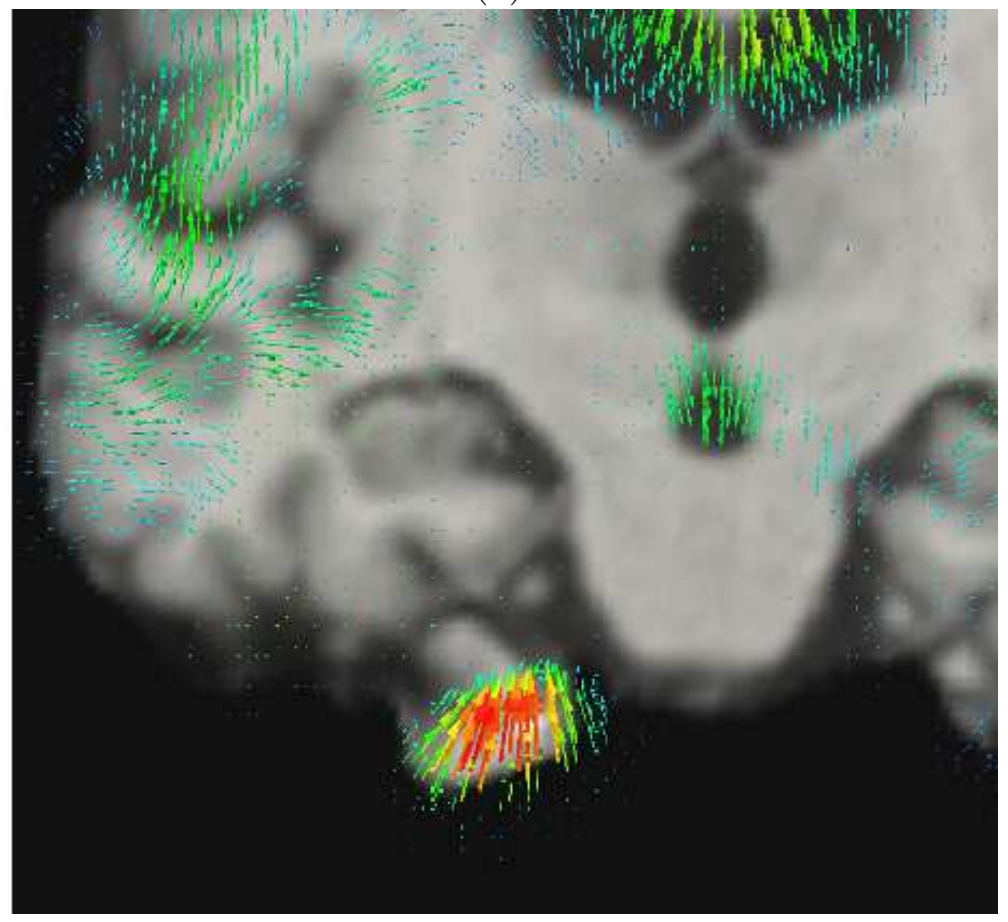

(b)

Figure 4.20: False deformation caused by the miss detection of topological change. 


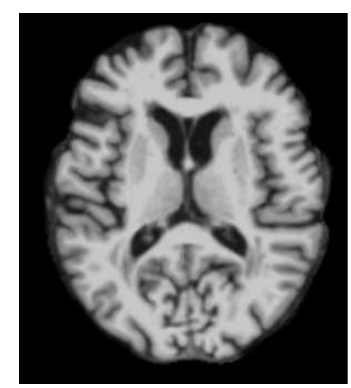

(a)

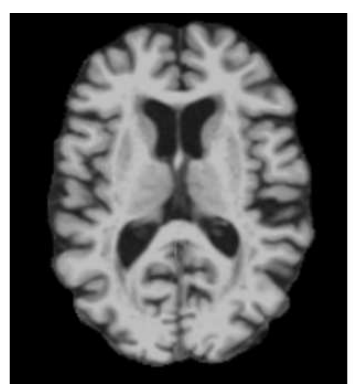

(b)

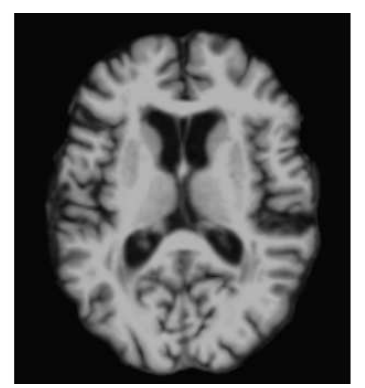

(c)

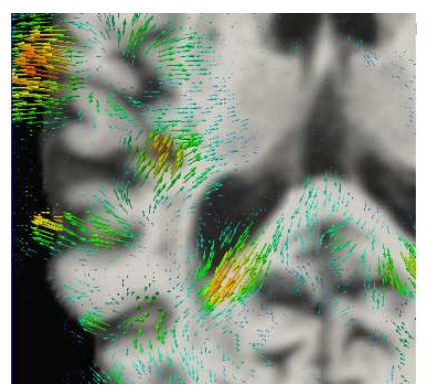

(d)

Figure 4.21: Sample registration when both moving and target image carry lesions. (a): moving image with lesion. (b): target image with lesion. (c): deformed moving image after spatial deformation. (d): the deformation field.

distributed within the Slicer3 [57] package. It was skull-stripped using the FSL (BET) tool[58]. As shown in the second row of Fig. 4.22, instead of a precise segmentation of the tumor, our interface allows a physician to draw 3 profile lines on the target image to indicate the location and size of the tumor (with orange line), which gives us an ellipsoid containing the tumor region (blue contours).

The third row of Fig. 4.22 gives the checkerboard image of the target image and the template; where the fourth row gives the checkerboard image of the target images and the registered template, after applying spatial deformation. We find that after registration, the template is well aligned with the target image. The fifth row shows the target image after applying intensity displacement, where we see the intensity of the tumor region is replaced by that of the co-aligned template image ${ }^{7}$. This step essentially provides us with a "tumor-repaired" version of the target image, which is an estimation of the brain anatomy had the tumor not existed. We notice that in the target image, the growth of tumor pushes the surrounding tissue aside and squeezes the ventricle. After intensity correction, the healthy tissue is moved back to the normal location and the ventricle is lifted. In the sixth row, we plot the resulting

\footnotetext{
${ }^{7}$ Note that in the checkerboard images in the third and fourth rows, the target image appears to be much darker compared to the template. The appearances of the target image before and after intensity correction, as shown in the second and fifth rows, are also very different. This is due to the fact that the target image has a very different intensity profile because of the bright tumor. As a result, the contrast of the whole image is not good, where CSF appears to be dark and not really visible. However, if we pay some close attention, it is easy to notice that major features, such as the separation between gray matter and white matter and the boundary of ventricle are all well aligned after the deformable registration. Also, as shown in the fifth row, after the intensity of the tumor region is repaired, brain regions other than the tumor can be visualized with a proper intensity contrast.
} 

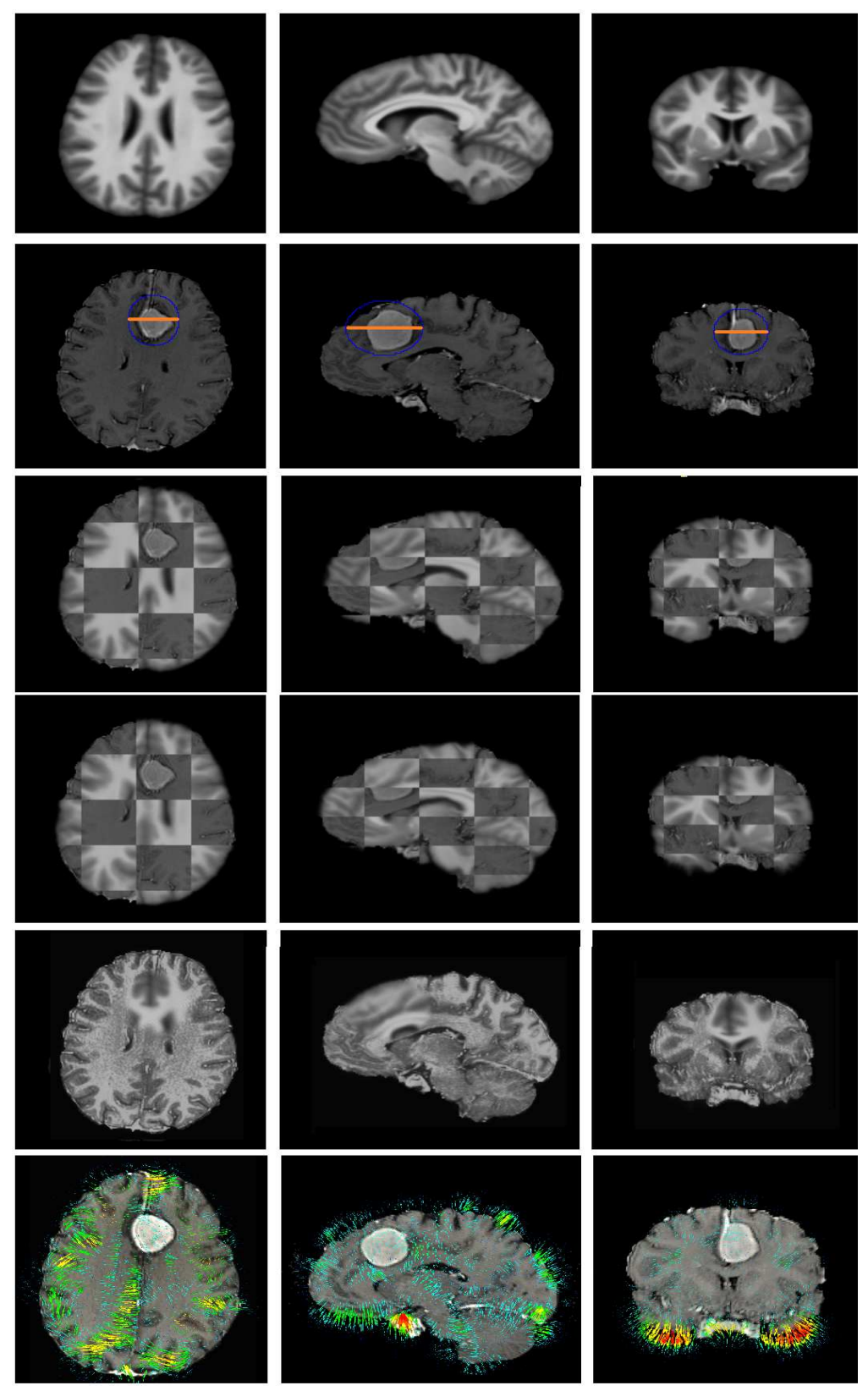

Figure 4.22: Sample registration of a brain template to the MRI of a meningioma patient. First row: a brain template, the moving image. Second row: target image, MRI of a meningioma patient. Third and fourth row: checkerboard image of the target and the moving image, before and after spatial deformation, respectively. The fifth row: the intensity corrected target image after intensity displacement. The sixth row: glyph plot of the resulting deformation field. The 3 columns show the Axial, Sagittal and Coronal views of the same image. Note that the length of glyph is amplified for better visualization and is not in real pixel scale. 
deformation field using glyphs. Clearly, the tumor does not induce any false deformation, despite the strong intensity gradient between the tumor region and the surrounding healthy tissue. In view of these results, we believe that the proposed algorithm might also be used to register images of resections as well.

\subsubsection{Registering images of normal topology.}

We need to point out that although our algorithm is motivated by the need for accurately registering images with topological changes, our algorithm can also be used to register images of normal topology, i.e., those without topological changes. The spatial deformation, in this case, contains all the deformation energy by setting the lesion probability map $p(x, y, z)$ to zero everywhere and does not allow the intensity to deform.

In Fig. 4.23 to Fig. 4.26 we give 4 examples using our registration algorithm, where both the moving and the target images have normal topology. In Fig. 4.23 to Fig. 4.26, the three columns give the Axial, Sagittal and Coronal views of the images. The first shows the checkerboard image of the target image and the template; and the second row shows the checkerboard image of the target image and the deformed template, i.e., after applying spatial deformation to the template. The third and fourth rows plot the iso-contours of the moving image before and after applying the spatial deformation, on top of the target image. Fig. 4.23 and Fig. 4.24 each shows the result of registering an elderly brain template, the same as used in Sec. 4.4.1, to a young and healthy subject from the OASIS dataset (age 27 and 21). Note that the brains of young subjects are of significant structural difference from the elderly template, which is particularly noticeable in terms of the ventricle sizes. In both cases the algorithm converges to very good alignments. Fig. 4.25 shows the results of registering the elderly brain template to an elderly healthy subject in OASIS, the same as used in Sec. 4.4.2. Fig. 4.26 shows the result of registering the image of the subject used in Fig. 4.23(b) to the subject used in Fig. 4.24(a). In all the cases, no intensity displacement was allowed, and thus the spatial deformation carries all the deformation energy.

The parameters used in template-to-subject registrations, i.e., Fig. 4.23 to Fig. 4.25 are set the same as used in Sec. 4.4.1 and those used in subject-to-subject registration, i.e., Fig. 4.26, are set the same as in Sec. 4.4.2. 

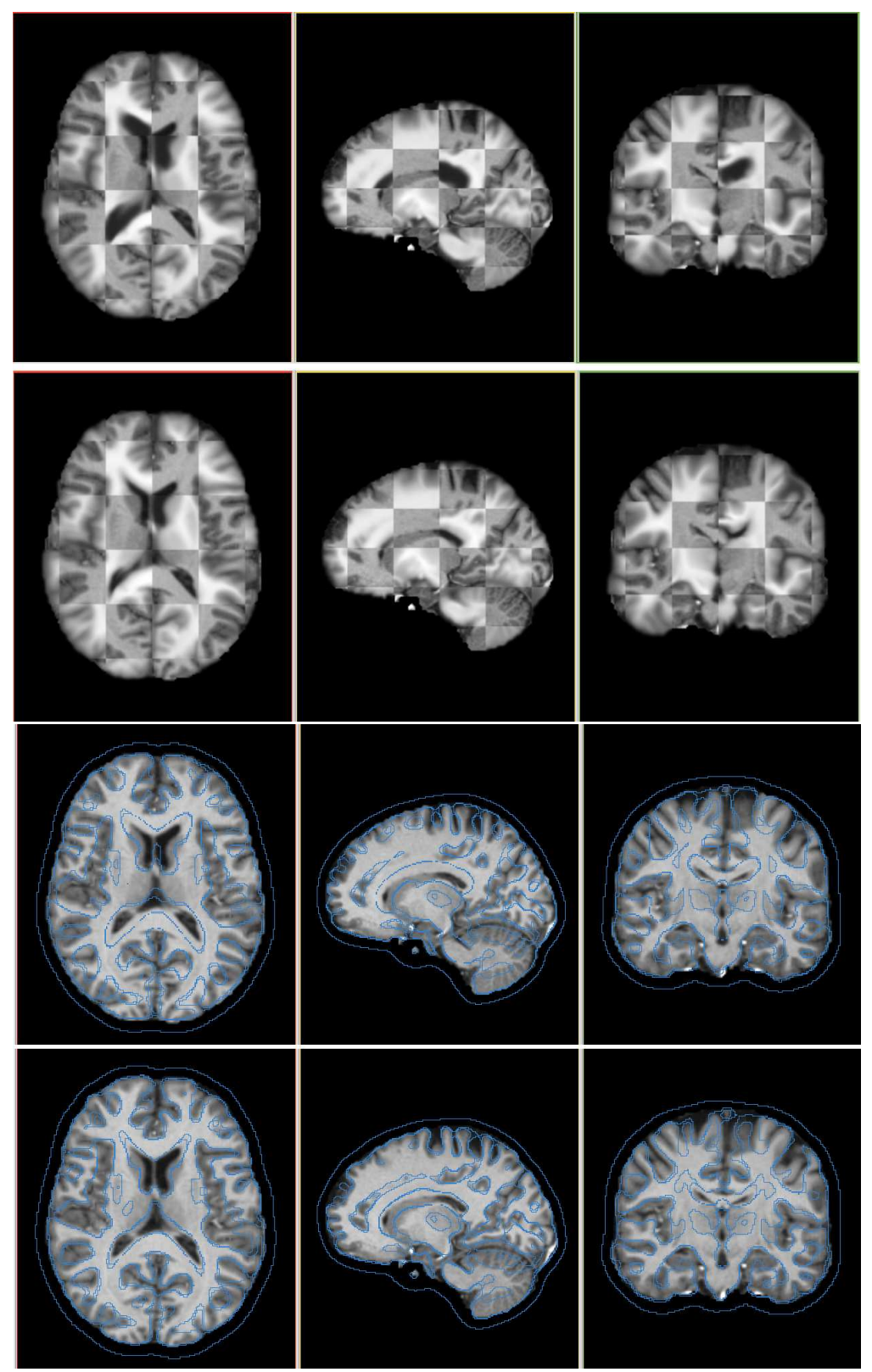

Figure 4.23: Sample result of registering the elderly template to a young and healthy subject from OASIS. The first and second rows give the checkerboard image of the target image and the template, before and after applying spatial deformation, respectively. The third and fourth row plot the iso-contours of the template, before and after registration, respectively, on top of the target image. 

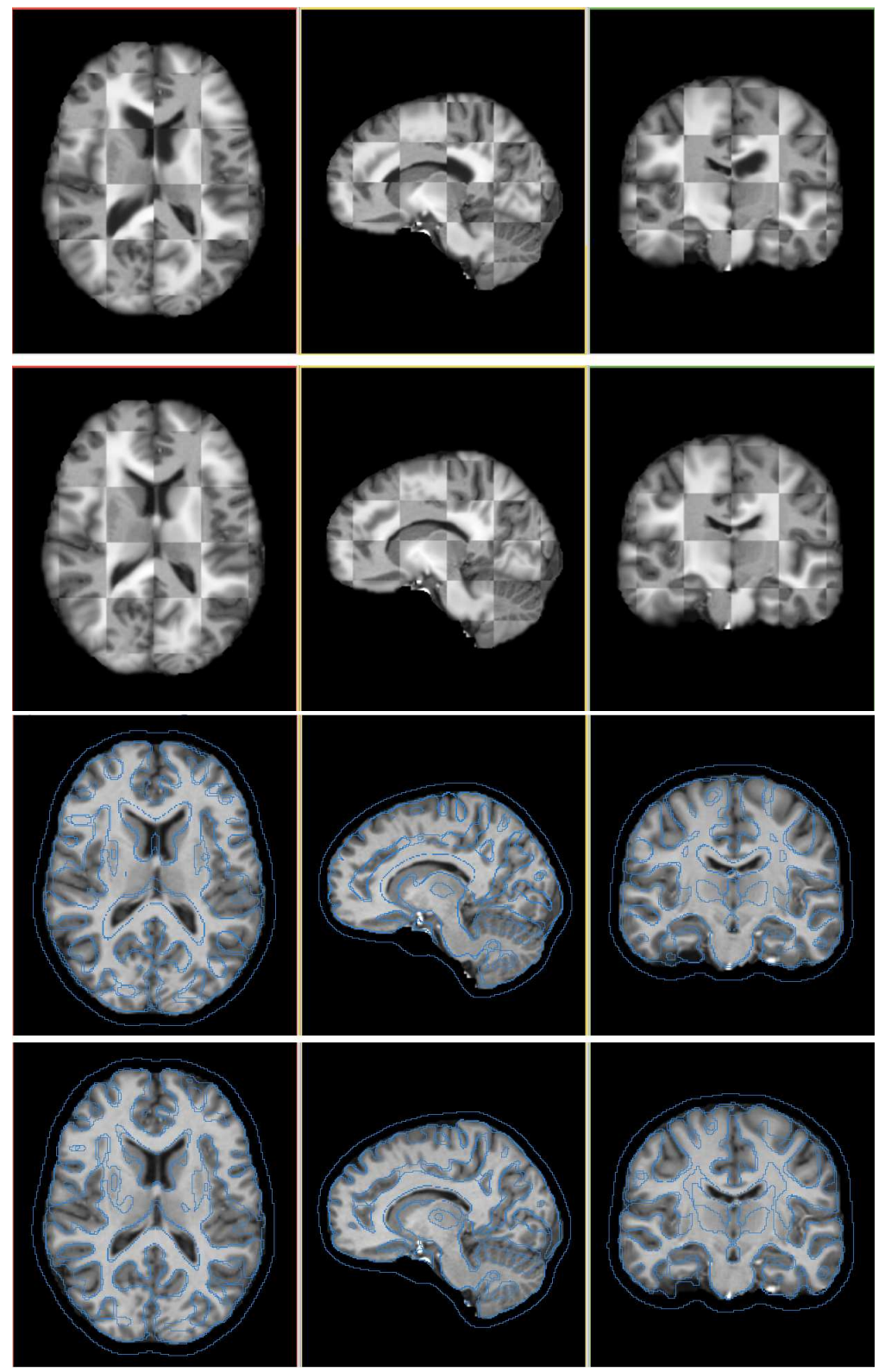

Figure 4.24: Another sample of registering the elderly template to a young and healthy subject from OASIS. Subfigures are organized in the same way as in Fig. 4.23. 

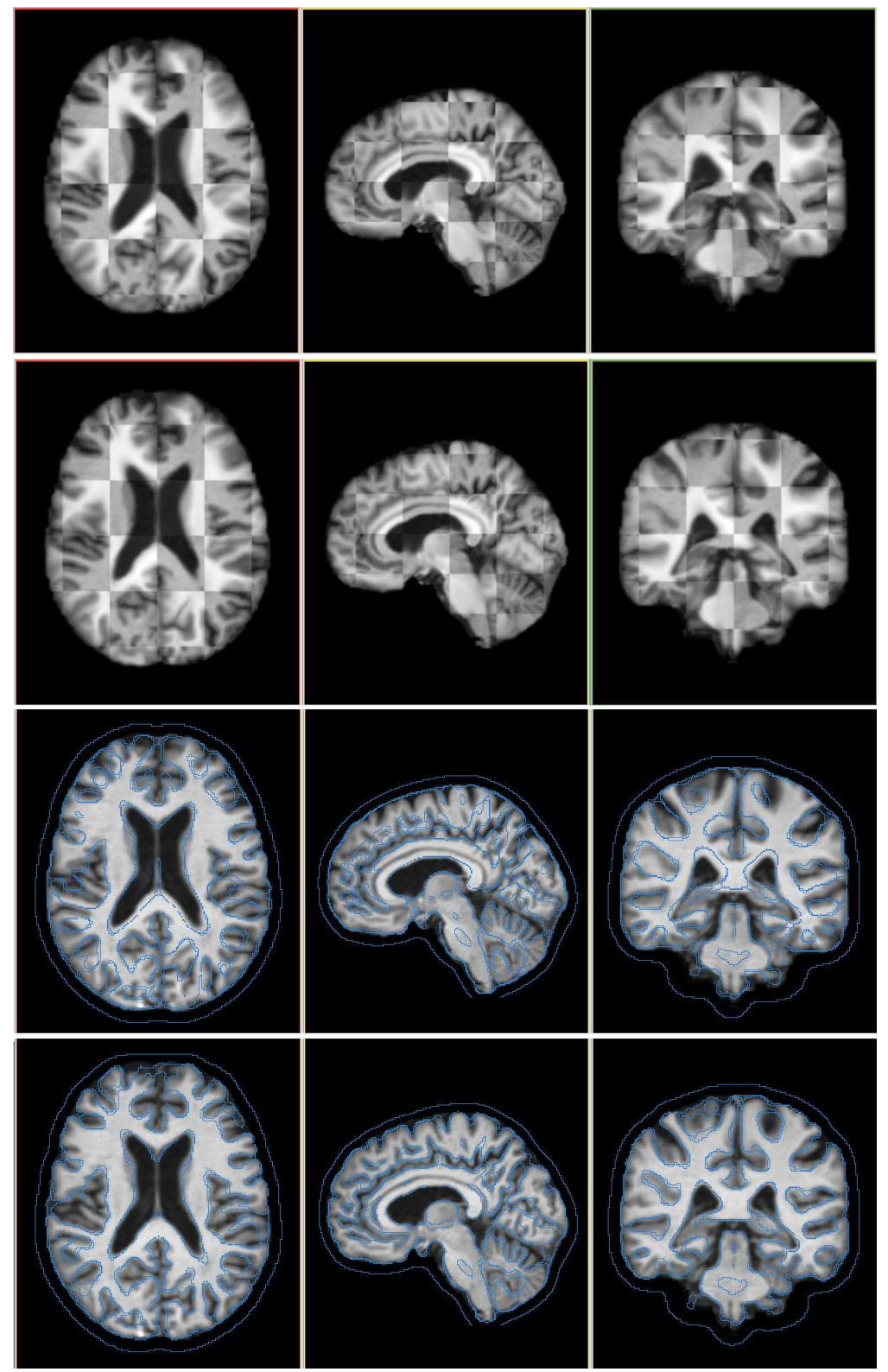

Figure 4.25: Sample result of registering the elderly template to a healthy elderly subject from OASIS. Subfigures are organized in the same way as in Fig. 4.23. 


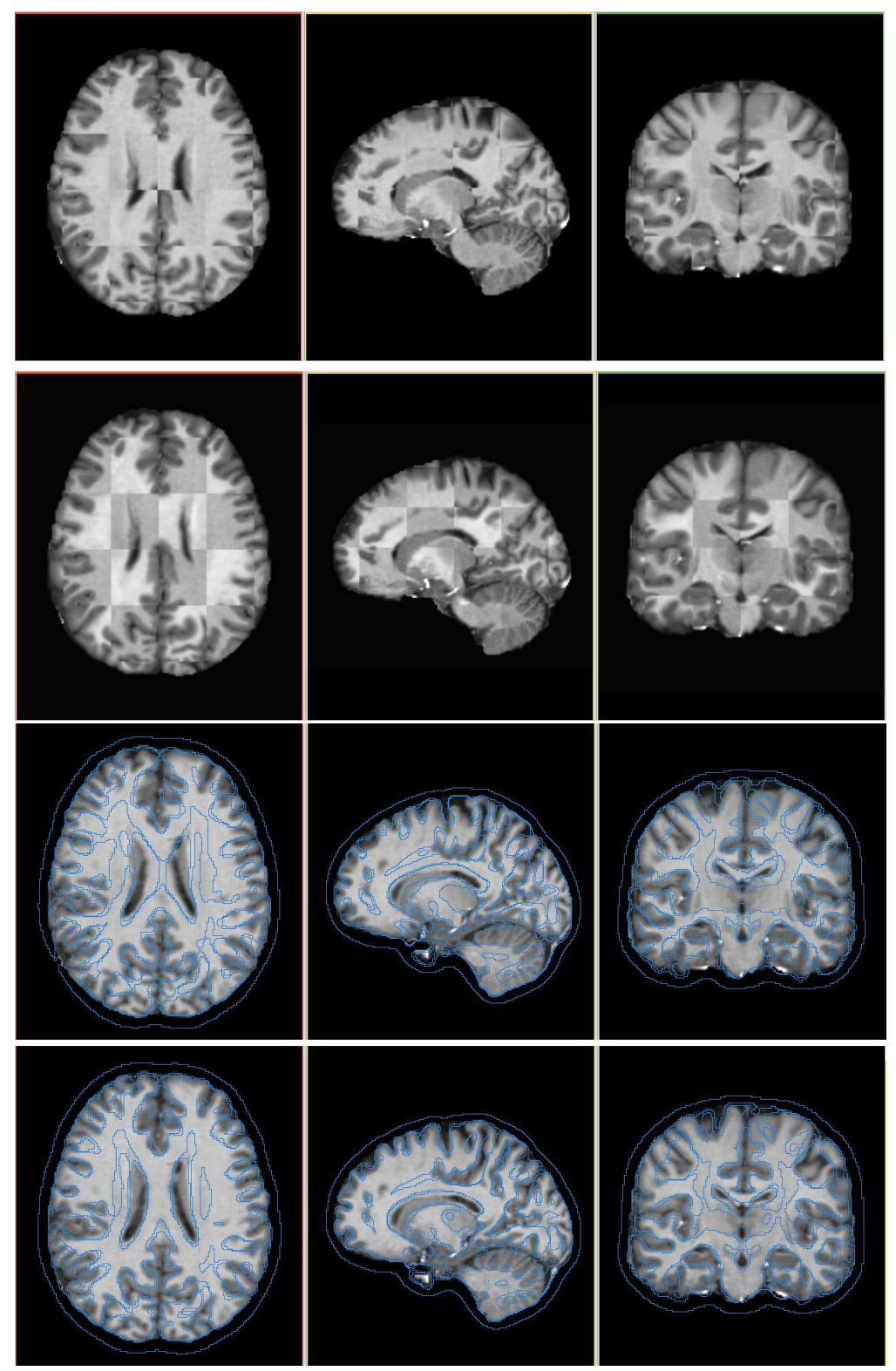

Figure 4.26: Sample result of registering a young and healthy subject from OASIS to another. Subfigures are organized in the same way as in Fig. 4.23. 


\subsubsection{Smoothness of $\beta$.}

In (4.13), we used a Gaussian kernel to construct the probability map by slightly blurring the boundary of the lesion segmentation. The purpose of this smoothing operation is to provide a smooth transition between the intensity corrected areas and their neighborhood. It also helps mitigate the adverse effect of imprecise segmentation, as discussed in Sec. 4.4.3. In clinical applications, this smoothing kernel should be selected based on the type of lesion, i.e., if the lesion of interest has a clear boundary for a precise segmentation, a small smoothing kernel can be used. On the other hand, for lesions that do not have a clear boundary, a larger smoothing kernel should be used. Fig. 4.28 provides an example showing the impact of the smoothing kernel. In particular, we conduct two experiments, where the elderly template, the same as used in Sec. 4.4.1, is registered to a brain image with lesions in the OASIS dataset, where $\sigma_{\text {prob }}$, for $K_{\text {prob }}$, is set to be 0 and 1 in the two experiments, respectively. The first row in Fig. 4.28(a) shows the checkerboard image of the template and the target image before registration. The second and third rows show the checkerboard images of the target image and the registered template after applying spatial deformation, using $\sigma_{\text {prob }}=0$ and $\sigma_{\text {prob }}=1$, respectively. All other parameters are kept the same as those used in Sec. 4.4.1. From Fig. 4.28, we can see the registration algorithm converges to very similar spatial deformation in the two experiments. However, the intensity displacement was affected by the choice of $\sigma_{\text {prob. }}$. This can be visualized in Fig. 4.28(b). In particular, the first row of Fig. 4.28(b) shows a zoomed view of the region with lesion in the target image, where the boundary of FreeSurfer lesion segmentation was marked by blue contours. The second and third rows show the lesion-repaired target images using $\sigma_{\text {prob }}=0$ and $\sigma_{\text {prob }}=1$, respectively. From the second row, we observe that there exists an artifact resulting from lesion-repair along the boundary of the lesion segmentation. This artifact, however, is mitigated by smoothing the label map, i.e., $\sigma_{\text {prob }}=1$, as can be seen in the third row. Also, even in the case of $\sigma_{\text {prob }}=0$, the sharp boundary in $p(x, y, z)$ does not show a strong impact on the diffusion process. This can be observed in Fig. 4.27, which shows the image $L_{2}$ residual during the two experiments. We see that image residuals in the two experiments decrease in a very similar fashion.

\subsubsection{Comparison with in-painting.}

As we discussed in Chapter 1, many current DBM methods perform a additional step of in-

painting prior to the deformable registration. Specifically, the brain MRI is first segmented into multiple classes. The classes corresponding to lesions are merged into proper anatomical 


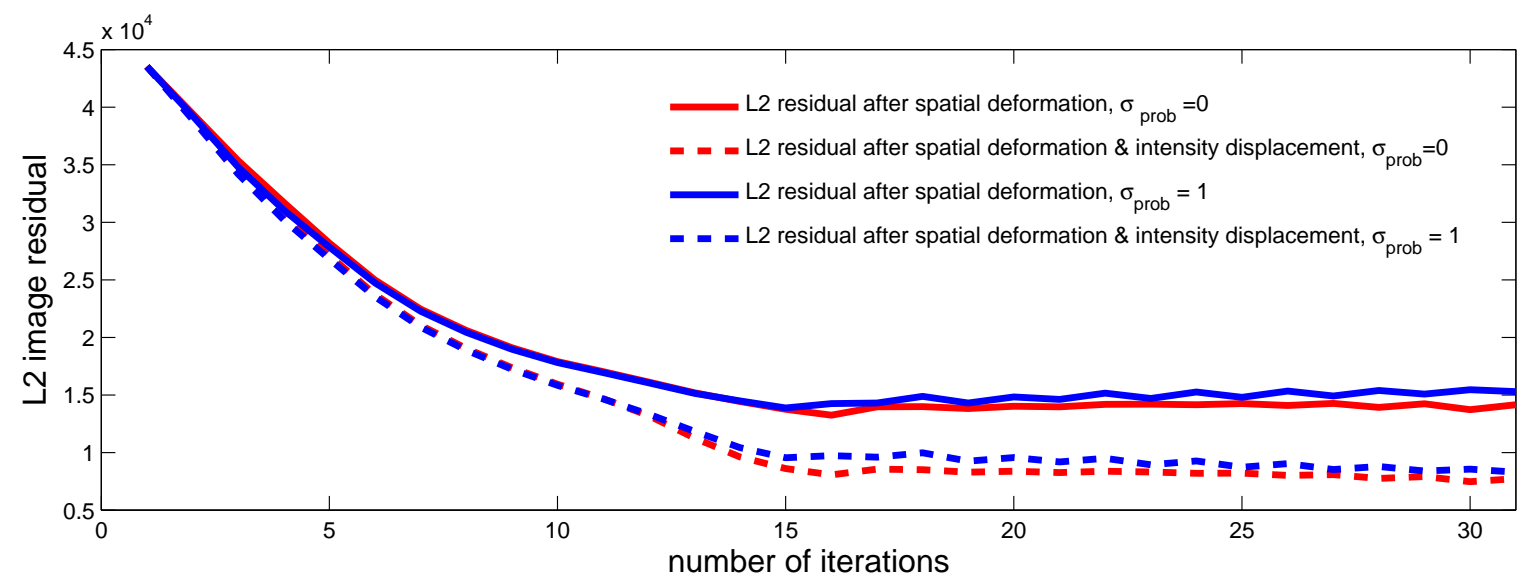

Figure 4.27: Curves plotting image $L_{2}$ residuals during the registration with $\sigma_{\text {prob }}=0$ and $\sigma_{\text {prob }}=1$.

structures, e.g., white matter lesions will be merged into white matter. Then, the segments of each class will be in-painted by the average intensity on the entire class.

In order to compare with the performance of in-painting, we tried to register a young and healthy subject, as shown in the first row in Fig. 4.29, to a elderly subject with lesion, as shown in the second row of Fig. 4.29, where both images are from the ADNI dataset. The moving image is first registered to the target image using our algorithm, where the deformed moving image is shown in the third row of Fig. 4.29. Then, we perform in-painting on both the moving and the target image based on the segmentation results obtained using the FreeSurfer tool. These in-painting results are shown in the first and second rows in Fig. 4.30. The moving and target images after in-painting are then registered using diffeomorphic demons, where the deformed moving image is shown in the third row of Fig. 4.30. Comparing the corresponding rows in Fig. 4.29 and Fig. 4.30, we find that the deformable registration algorithms converge to reasonable results in both cases.

In Fig. 4.31(a), we show the glyph views of the deformation fields obtained through diffeomorphic demons after in-painting (left) and our registration algorithm (right). We find that in both cases, the lesions around the lateral ventricle did not induce false deformation. However, when we apply the deformation field obtained through diffeomorphic demons after in-painting on the original moving image, we notice certain artifacts with blocks of uniform intensities, as shown in the zoomed images in the upper row of Fig. 4.31(b). This is not seen 

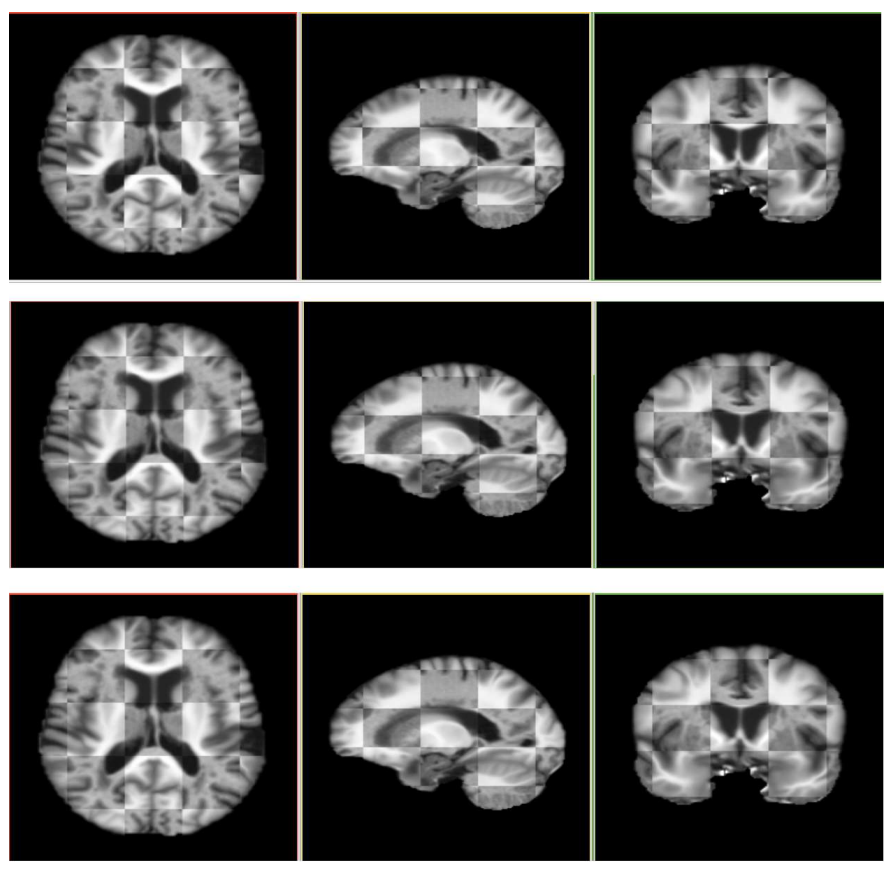

(a)
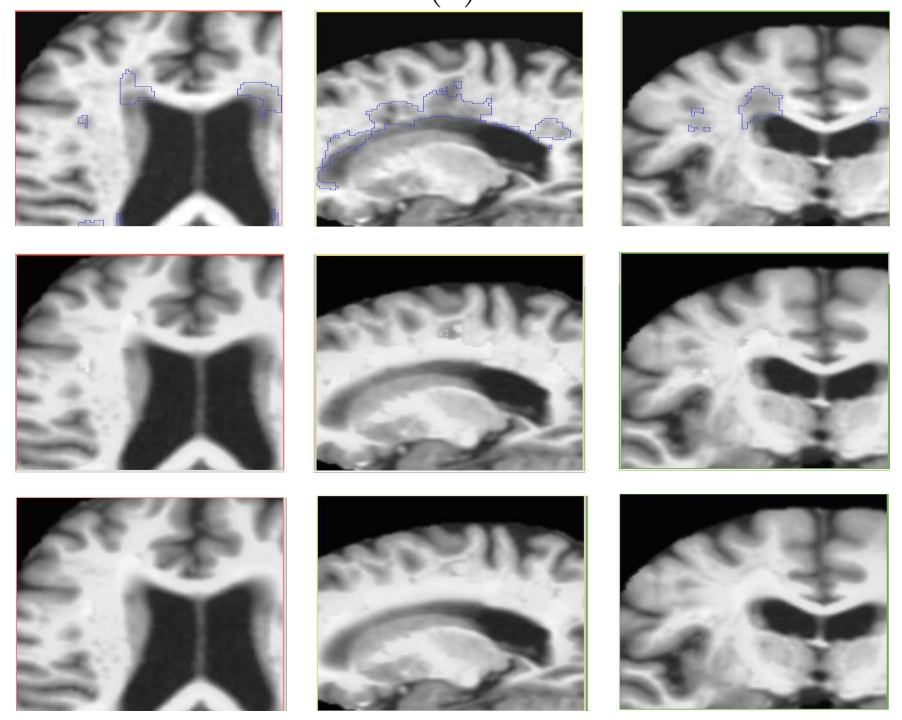

(b)

Figure 4.28: Example showing the impact of smoothing kernel $K_{\text {prob }}$. (a): The first row shows the checkerboard image of the template and target image before registration. The second and third row show the registered template after applying spatial deformation, using $\sigma_{\text {prob }}=0$ and $\sigma_{\text {prob }}=1$, respectively. (b): the first row shows a zoom-in view of the region with lesion in the target image, where the boundary of FreeSurfer lesion segmentation was marked by blue contours. The second and third rows show the lesion-repaired target images using $\sigma_{\text {prob }}=0$ and $\sigma_{\text {prob }}=1$, respectively. 
in the result of our algorithm, as in the lower row of Fig. 4.31(b). Clearly, after in-painting, the deformable registration algorithm is essentially registering image blocks (segments) of uniform intensities. The artifact is a consequence of this block-wise registration. We believe this provides some evidence that our registration algorithm maybe capable of capturing more detailed shape difference, compared with the matching between in-painted blocks. 

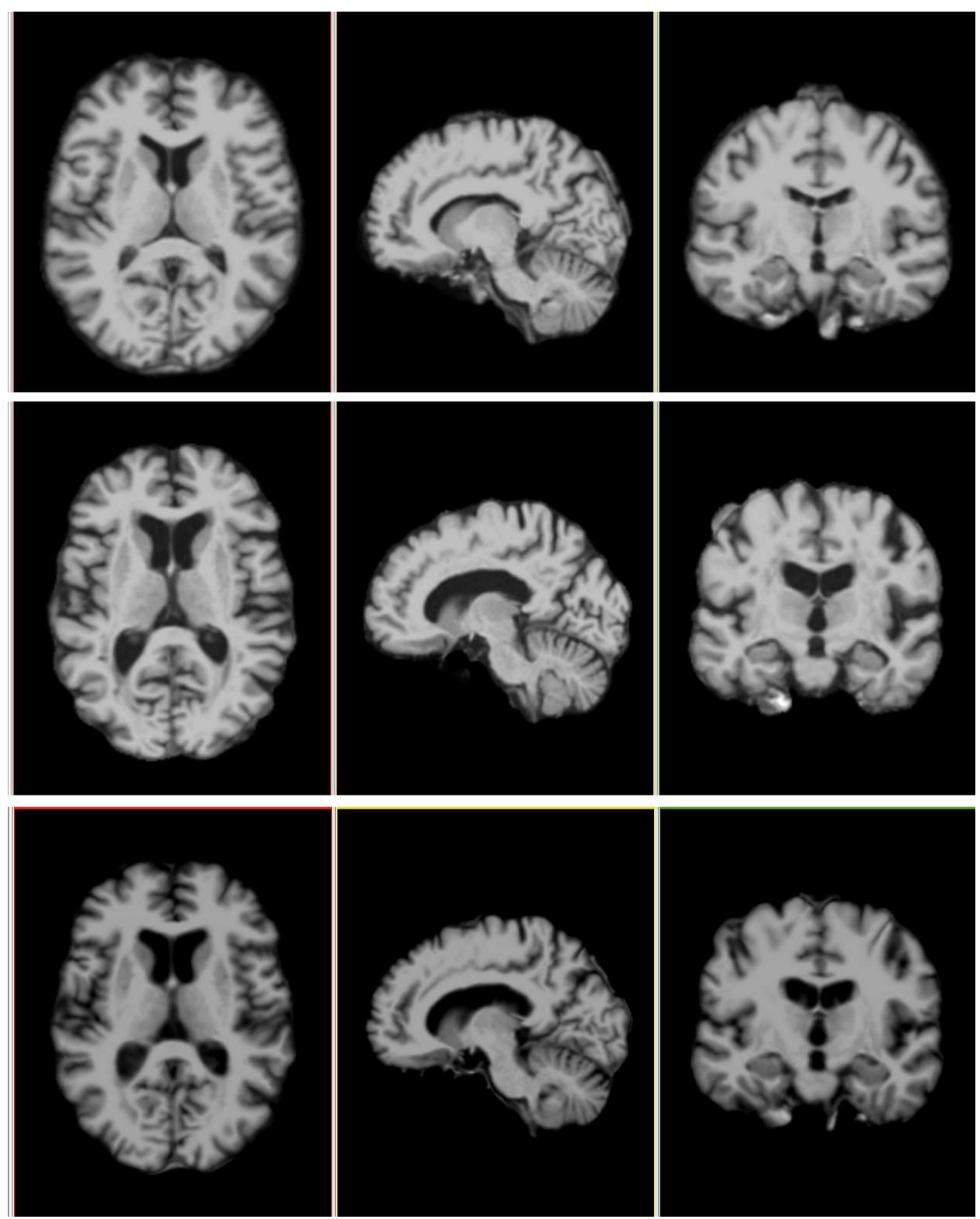

Figure 4.29: Sample registration result using our algorithm. First row: moving image. Second: target image. Third row: spatial deformed moving image. 

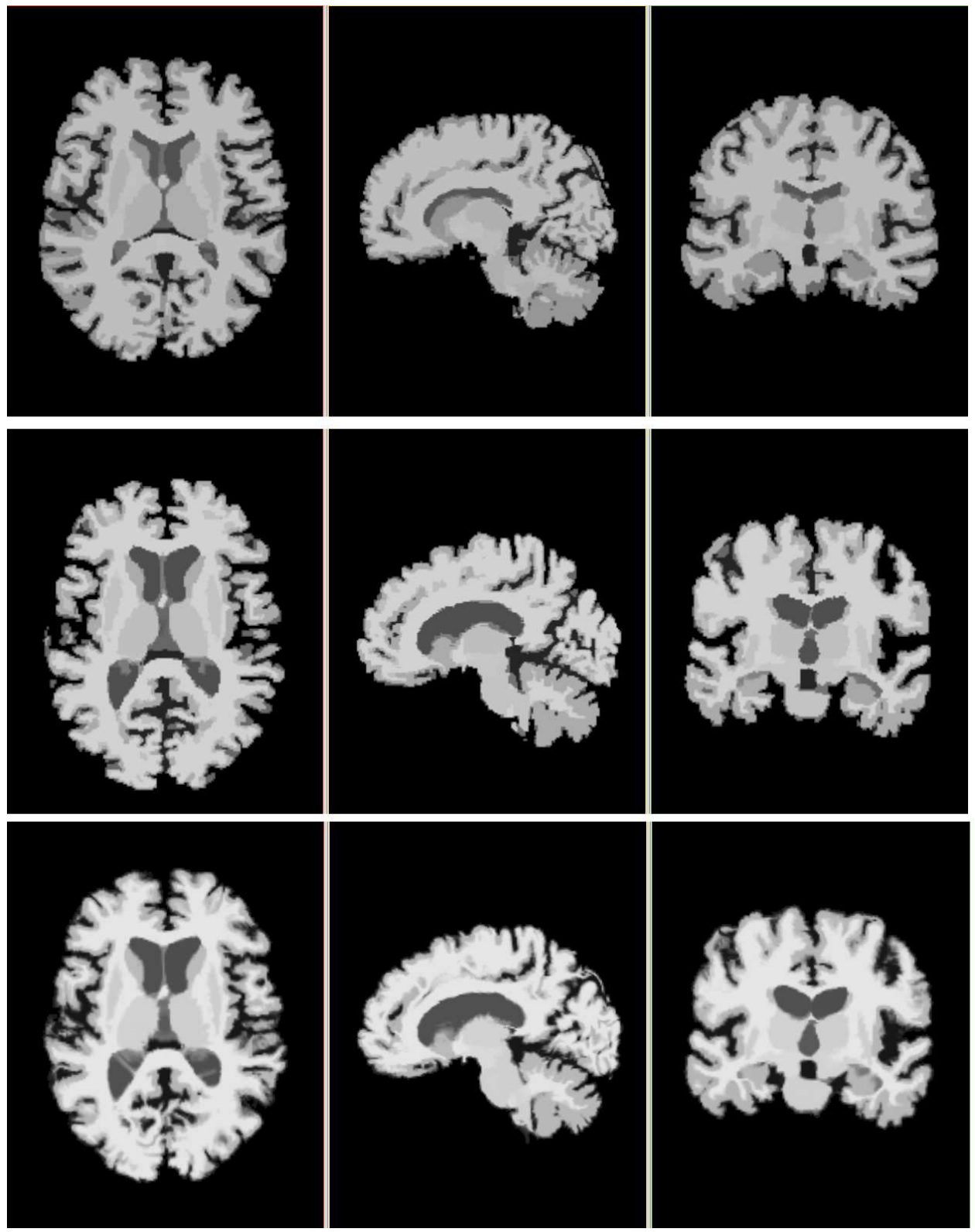

Figure 4.30: On the same registration experiment as in Fig. 4.29, registration result when in-painting is used. First row: moving image after in-painting. Second: target image after in-painting. Third row: spatial deformed moving image, i.e., after apply the deformation field obtained using diffeomorphic demons on the in-painted image. 

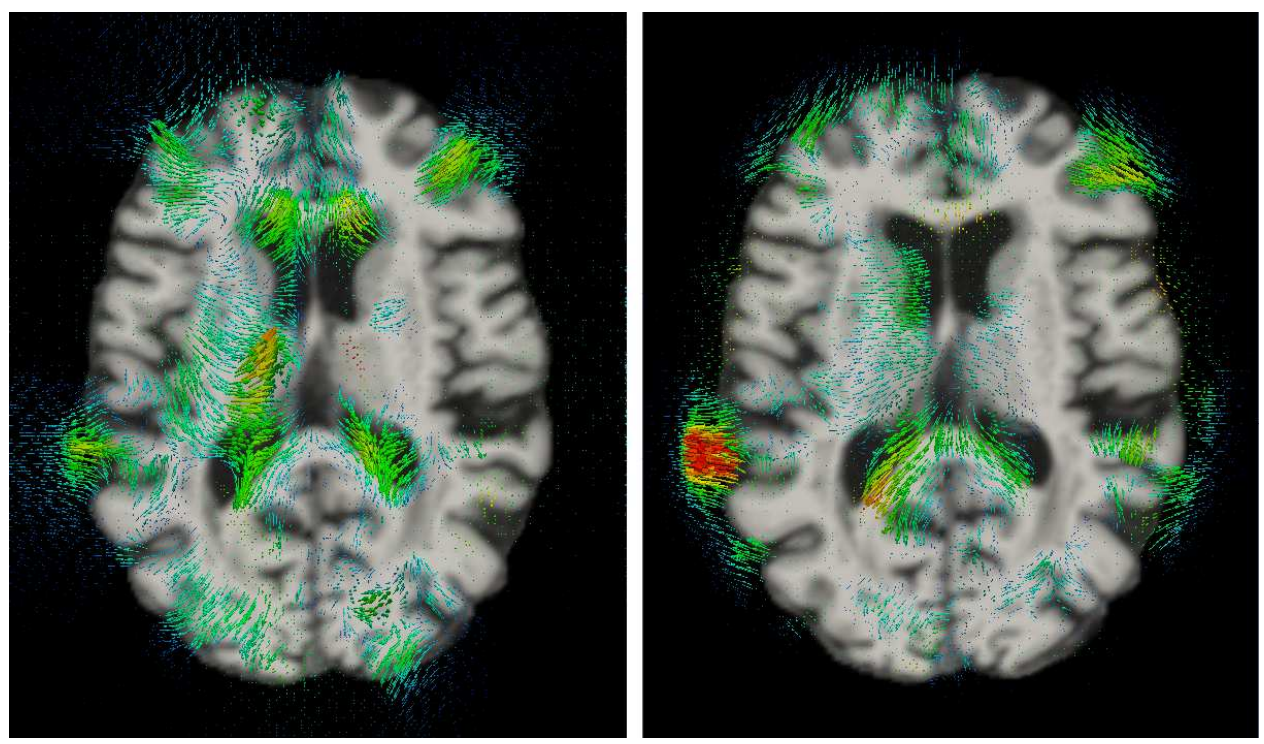

(a)
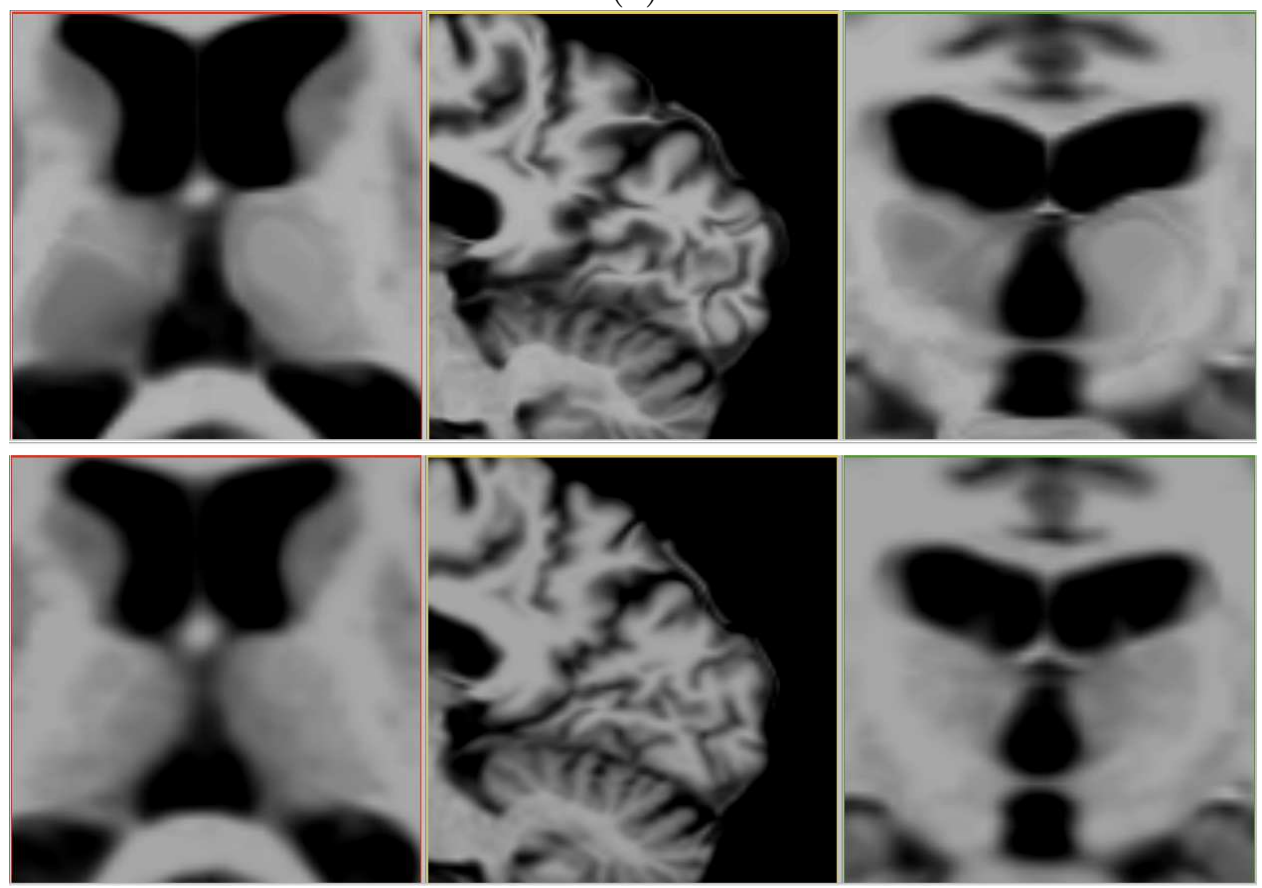

(b)

Figure 4.31: Impact of in-painting. (a) glyph view of the deformation field obtained using diffeomorphic demons after in-painting (left) and our algorithm (right). (b) upper row: result when the deformation field obtained using in-painting is applied on the original moving image; lower row: result when the deformation field obtained using our algorithm is applied on the original moving image. 


\section{Chapter 5}

\section{Conclusion and Future Work}

Deformable registration is an important research field in medical image processing, since the resulting dense deformation fields provide rich information for clinical and basic science studies. As demonstrated in Chapter 3, topological changes cause false deformation and degrade the reliability of existing deformable registration algorithms. This motivated the development of a deformable registration algorithm that is capable of registering images with topological changes, eliminating false deformation. We now summarize our contributions in tackling this research problem.

\section{$5.1 \quad$ Research summary}

In this work, we first study the impact of topological changes on existing deformable registration algorithms, which demonstrates the necessity of suppressing false deformation. To handle the issue, we develop a new deformable registration algorithm for images with topological changes. The proposed algorithm is capable of using a segmentation of the topological changes as an additional input to eliminate false deformation in the resulting deformation field. In particular, the registration is performed by embedding images in $\mathbb{R}^{3}$ Euclidian space into surfaces in an $\mathbb{R}^{4}$ Riemannian space. Then the image registration is modeled as a surface evolution process. Using the information provided by the a-priori segmentation, our algorithm controls the distribution of the deformation energy between the spatial deformation and intensity displacement. By doing so, false deformation is effectively suppressed, and the intensity displacement, as a by-product of our algorithm, captures the intensity difference 
caused by the topological change. The algorithm was extensively tested on different brain MR image datasets containing minor to severe pathology. The registration results are compared with those obtained from diffeomorphic demons, a representative modern algorithm used in neuroimaging studies. The comparison demonstrates the efficacy of our proposed algorithm in terms of converging to a resonable registration in the presence of topological changes. More importantly, the resulting deformation field is free of false deformation, which correctly reveals the structural differences between imaged brains.

The largest impact we foresee of the algorithm is an improved accuracy of DBM analysis in the researches that has to deal frequently with lesions, including studies of aging, lupus, MS, and Alzheimer's. As shown in Sec. 4.4.7, our proposed algorithm avoids the extra step of in-painting that is commonly used in DBM, and is believed to capture more detailed shape differences, compared with the matching between in-painted blocks. In addition, our registration algorithm also outputs a lesion correction result, which repairs the intensity of topological changes in the input image. This by-product can also be useful in many clinical studies, such as template building.

We also highlight the value of our proposed algorithm in terms of ease to implement, robustness, fast convergence, intuitive parameter settings and limited memory space consumption (around 1/40 of that required by the original LDDMM). As shown in Sec. 4.4.5, our proposed algorithm is also capable of registering images with normal topology, which proves that our method is a generally applicable registration tool. Considering all these merits, we believe our proposed algorithm will find its application in many large-scale group-wise or longitudinal brain studies.

\section{$5.2 \quad$ Future work}

The current version of our implementation only allows the target image to contain a topological change relative to the source image. A direct extension would be to symmetrize the energy function and allow lesions in both the moving and the target images to be modeled by separate probability maps. If mutual information is used instead of the $L_{2}$ image residual in the embedding function $\phi$, our proposed algorithm might also register images across different modalities. In addition, as shown in Sec. 4.4.3, our algorithm can also be used to register brain images that are not-skull stripped, where the brain mask can be modeled in $p$.

In the future, the proposed registration algorithms can be integrated into the pipeline of 
studies related to group-wise analysis, or longitudinal study of disease progression. In our research group, an automatic unsupervised classification algorithm is currently being developed to distinguish brain MR images of AD patients from those of elderly normal controls. The classification uses the metric computed from dense registration vector fields, an example result of which is illustrated by Fig. 5.1. However, the issue of false deformation with existing deformable registration algorithms degrades the classification performance. Ideally, the structural difference and intensity difference caused by lesions among brains are two difference sources in the analysis of brain variations. Our proposed registration algorithm is well suited for this application, where it can provide two separate metrics for such classification algorithms: one is the spatial deformation field for brain structural changes that is free of false deformation, and the other is the intensity displacement which contains only the intensity difference caused by lesions. This well-separated and enriched information is expected to enhance the performance of classification and provide new information for severe disease studies. 


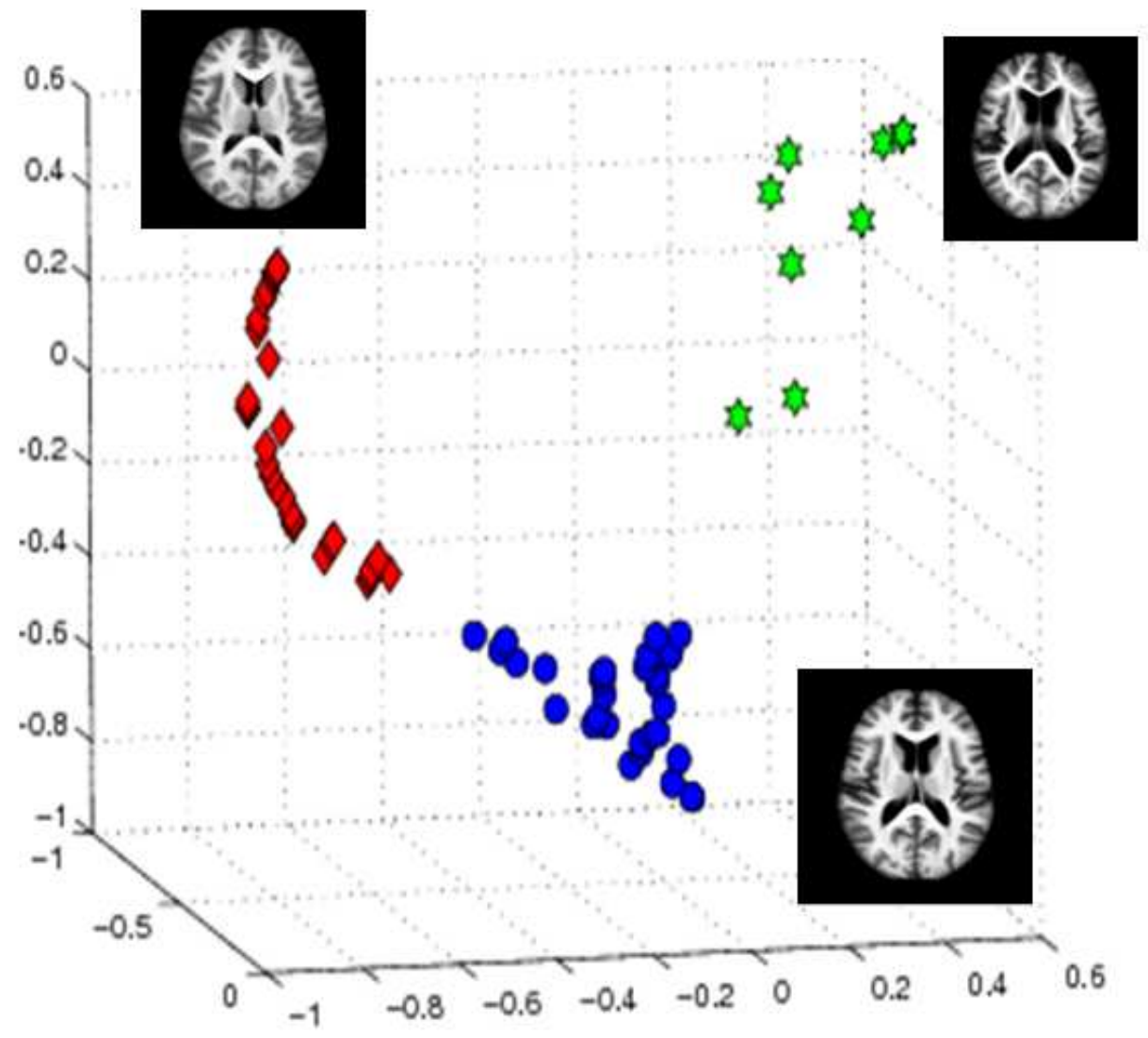

Figure 5.1: Classification on dataset using whole-brain distances. Quick shift classification result in the embedded space [2]. 


\section{Appendix A}

\section{Derivation of Euler-Lagrange equation}

Given an image manifold and its metric $(\Sigma, g)$ in $\mathbb{R}^{3}$, we embed it as feature-scale manifold $(M, h)$ in $\mathbb{R}^{4}$ through the map $X: \Sigma \rightarrow M$ :

$$
X=[x, y, z, I(x, y, z)],
$$

where $I$ is the image intensity feature, which is a spatial varying function with respect to the image local corrdinates $x, y, z$.

To get a conformal area of the embedded surface that is approprate for a registration objective, we choose the following metirc in $M$ :

$$
h=\left[\begin{array}{cccc}
\phi & 0 & 0 & 0 \\
0 & \phi & 0 & 0 \\
0 & 0 & \phi & 0 \\
0 & 0 & 0 & \beta \phi
\end{array}\right]
$$

where $\phi(x, y, z, I)$ is a chosen embedding function. $\beta(x, y, z)$ is a scaling function that controls the relative magnitude of the embedded scale-space. The elements in $h$ are indexed as $h_{i, j}, i, j=1, \ldots, 4$. The inverse of $h$ can be directly obtained: 


$$
h^{-1}=\left[\begin{array}{cccc}
\frac{1}{\phi} & 0 & 0 & 0 \\
0 & \frac{1}{\phi} & 0 & 0 \\
0 & 0 & \frac{1}{\phi} & 0 \\
0 & 0 & 0 & \frac{1}{\beta \phi}
\end{array}\right]
$$

whose elements are indexed as $h^{i j}, i, j=1, \ldots, 4$.

Now we compute the metric $g$ through the pullback procedure:

$$
g_{\mu \nu}=h_{i j} \partial_{\mu} X^{i} \partial_{\nu} X^{j}
$$

Note that Einstein summation convention is used, with $\mu, \nu=1,2,3$ and $i, j=1, \ldots, 4$.

$$
\begin{aligned}
& g_{11}=h_{11} \partial_{x} X^{1} \partial_{x} X^{1}+h_{44} \partial_{x} X^{4} \partial_{x} X^{4}=\phi\left(1+\beta I_{x}^{2}\right) ; \\
& g_{12}=h_{44} \partial_{x} X^{4} \partial_{y} X^{4}=\phi \beta I_{x} I_{y} ; \\
& g_{13}=h_{44} \partial_{x} X^{4} \partial_{z} X^{4}=\phi \beta I_{x} I_{z} ; \\
& g_{21}=h_{44} \partial_{y} X^{4} \partial_{x} X^{4}=\phi \beta I_{x} I_{y} ; \\
& g_{22}=h_{22} \partial_{y} X^{2} \partial_{y} X^{2}+h_{44} \partial_{y} X^{4} \partial_{y} X^{4}=\phi\left(1+\beta I_{y}^{2}\right) ; \\
& g_{23}=h_{44} \partial_{y} X^{4} \partial_{z} X^{4}=\phi \beta I_{y} I_{z} ; \\
& g_{31}=h_{44} \partial_{z} X^{4} \partial_{x} X^{4}=\phi \beta I_{x} I_{z} ; \\
& g_{32}=h_{44} \partial_{z} X^{4} \partial_{y} X^{4}=\phi \beta I_{y} I_{z} ; \\
& g_{33}=h_{33} \partial_{z} X^{2} \partial_{z} X^{2}+h_{44} \partial_{z} X^{4} \partial_{z} X^{4}=\phi\left(1+\beta I_{z}^{2}\right) ;
\end{aligned}
$$

thus, we have

$$
g=\phi\left[\begin{array}{ccc}
1+\beta I_{x}^{2} & \beta I_{x} I_{y} & \beta I_{x} I_{z} \\
\beta I_{x} I_{y} & 1+\beta I_{y}^{2} & \beta I_{y} I_{z} \\
\beta I_{x} I_{z} & \beta I_{x} I_{z} & 1+\beta I_{z}^{2}
\end{array}\right]
$$

The determinant of $g$ is computed as: 


$$
\begin{aligned}
|g| & =\phi^{3}\left[\left(1+\beta I_{x}^{2}\right)\left(1+\beta I_{y}^{2}\right)\left(1+\beta I_{z}^{2}\right)+2 \beta^{3} I_{x}^{2} I_{y}^{2} I_{z}^{2}-\beta^{3} I_{x}^{2} I_{z}^{2}\right. \\
& -\beta^{3} I_{x}^{2} I_{y}^{2} I_{z}^{2}-\beta^{3} I_{y}^{2} I_{z}^{2}-\beta^{3} I_{x}^{2} I_{y}^{2} I_{z}^{2}-\beta^{3} I_{x}^{2} I_{y}^{2}-\beta^{3} I_{x}^{2} I_{y}^{2} I_{z}^{2} \\
& =\phi^{3}\left(1+\beta I_{x}^{2}+\beta I_{y}^{2}+\beta I_{z}^{2}\right) .
\end{aligned}
$$

Then, we get the inverse of $g$ :

$$
\begin{aligned}
& g_{22} g_{33}-g_{32} g_{23}=\phi^{2}+\beta \phi^{2}\left(I_{y}^{2}+I_{z}^{2}\right)+\beta^{2} \phi^{2}\left(I_{y}^{2} I_{z}^{2}-I_{y}^{2} I_{z}^{2}\right)=\phi^{2}\left(1+\beta I_{y}^{2}+\beta I_{z}^{2}\right) ; \\
& g_{13} g_{32}-g_{12} g_{33}=\beta^{2} \phi^{2} I_{x} I_{y} I_{z}^{2}-\beta \phi^{2} I_{x} I_{y}-\beta^{2} \phi^{2} I_{x} I_{y} I_{z}^{2}=-\beta \phi^{2} I_{x} I_{y} ; \\
& g_{12} g_{23}-g_{13} g_{22}=\beta^{2} \phi^{2} I_{x} I_{y}^{2} I_{z}-\beta \phi^{2} I_{x} I_{z}-\beta^{2} \phi^{2} I_{x} I_{y}^{2} I_{z}=-\beta \phi^{2} I_{x} I_{z} ; \\
& g_{23} g_{31}-g_{21} g_{33}=\beta^{2} \phi^{2} I_{x} I_{y} I_{z}^{2}-\beta \phi^{2} I_{x} I_{y}-\beta^{2} \phi^{2} I_{x} I_{y} I_{z}^{2}=-\beta \phi^{2} I_{x} I_{y} ; \\
& g_{11} g_{33}-g_{31} g_{13}=\phi^{2}+\beta \phi^{2}\left(I_{x}^{2}+I_{z}^{2}\right)+\beta^{2} \phi^{2}\left(I_{x}^{2} I_{z}^{2}-I_{x}^{2} I_{z}^{2}\right)=\phi^{2}\left(1+\beta I_{x}^{2}+\beta I_{z}^{2}\right) ; \\
& g_{13} g_{21}-g_{11} g_{23}=\beta^{2} \phi^{2} I_{x}^{2} I_{y} I_{z}-\beta \phi^{2} I_{y} I_{z}-\beta^{2} \phi^{2} I_{x}^{2} I_{y} I_{z}=-\beta \phi^{2} I_{y} I_{z} ; \\
& g_{21} g_{32}-g_{22} g_{31}=\beta^{2} \phi^{2} I_{x} I_{y}^{2} I_{z}-\beta \phi^{2} I_{x} I_{z}-\beta^{2} \phi^{2} I_{x} I_{y}^{2} I_{z}=-\beta \phi^{2} I_{x} I_{z} ; \\
& g_{12} g_{31}-g_{11} g_{32}=\beta^{2} \phi^{2} I_{x}^{2} I_{y} I_{z}-\beta \phi^{2} I_{y} I_{z}-\beta^{2} \phi^{2} I_{x}^{2} I_{y} I_{z}=-\beta \phi^{2} I_{y} I_{z} ; \\
& g_{11} g_{22}-g_{12} g_{21}=\phi^{2}+\beta \phi^{2}\left(I_{x}^{2}+I_{y}^{2}\right)+\beta^{2} \phi^{2}\left(I_{x}^{2} I_{y}^{2}-I_{x}^{2} I_{y}^{2}\right)=\phi^{2}\left(1+\beta I_{x}^{2}+\beta I_{y}^{2}\right) ;
\end{aligned}
$$

thus:

$$
g^{-1}=\frac{1}{\phi\left(1+\beta I_{x}^{2}+\beta I_{y}^{2}+\beta I_{z}^{2}\right)}\left[\begin{array}{ccc}
1+\beta I_{y}^{2}+\beta I_{z}^{2} & -\beta I_{x} I_{y} & -\beta I_{x} I_{z} \\
-\beta I_{x} I_{y} & 1+\beta I_{x}^{2}+\beta I_{z}^{2} & -\beta I_{y} I_{z} \\
-\beta I_{x} I_{z} & -\beta I_{x} I_{z} & 1+\beta I_{x}^{2}+\beta I_{y}^{2}
\end{array}\right]
$$

whose elements are indexed by $g^{\mu \nu}, \mu, \nu=1,2,3$. The evolution of the embedded surface is defined through the Euler-Lagrange equation of the conformal surface area measured by the Polykov Functional:

$$
\frac{\partial X^{i}}{\partial t}=\frac{\phi^{2}}{\sqrt{g}} \partial_{\mu}\left(\sqrt{g} g^{\mu \nu} \partial_{\nu} X^{i}\right)+\phi^{2} \Gamma_{j k}^{i} \partial_{\mu} X^{j} \partial_{\nu} X^{k} g^{\mu \nu},
$$

whose $\Gamma_{j k}^{i}$ are the Levi-Civita connection coefficients, computed as: 


$$
\Gamma_{j k}^{i}=\frac{1}{2} h^{i l}\left(\partial_{j} h_{l k}+\partial_{k} h_{j l}-\partial_{l} h_{j k}\right)
$$

so,

$$
\begin{aligned}
& \Gamma_{11}^{1}=\frac{1}{2} h^{11}\left(\partial_{x} h_{11}+\partial_{x} h_{11}-\partial_{x} h_{11}\right)=\frac{\phi_{x}}{2 \phi} \\
& \Gamma_{12}^{1}=\frac{1}{2} h^{11}\left(\partial_{x} h_{12}+\partial_{y} h_{11}-\partial_{x} h_{12}\right)=\frac{\phi_{y}}{2 \phi} \\
& \Gamma_{13}^{1}=\frac{1}{2} h^{11}\left(\partial_{x} h_{13}+\partial_{z} h_{11}-\partial_{x} h_{13}\right)=\frac{\phi_{z}}{2 \phi} \\
& \Gamma_{14}^{1}=\frac{1}{2} h^{11}\left(\partial_{x} h_{14}+\partial_{I} h_{11}-\partial_{x} h_{14}\right)=\frac{\phi_{I}}{2 \phi} \\
& \Gamma_{21}^{1}=\frac{1}{2} h^{11}\left(\partial_{y} h_{11}+\partial_{x} h_{21}-\partial_{x} h_{21}\right)=\frac{\phi_{y}}{2 \phi} \\
& \Gamma_{22}^{1}=\frac{1}{2} h^{11}\left(\partial_{y} h_{12}+\partial_{y} h_{21}-\partial_{x} h_{22}\right)=-\frac{\phi_{x}}{2 \phi} \\
& \Gamma_{23}^{1}=\frac{1}{2} h^{11}\left(\partial_{y} h_{13}+\partial_{z} h_{21}-\partial_{x} h_{23}\right)=0 \\
& \Gamma_{24}^{1}=\frac{1}{2} h^{11}\left(\partial_{y} h_{14}+\partial_{I} h_{21}-\partial_{x} h_{24}\right)=0 \\
& \Gamma_{31}^{1}=\frac{1}{2} h^{11}\left(\partial_{z} h_{11}+\partial_{x} h_{31}-\partial_{x} h_{31}\right)=\frac{\phi_{z}}{2 \phi} \\
& \Gamma_{32}^{1}=\frac{1}{2} h^{11}\left(\partial_{z} h_{12}+\partial_{y} h_{31}-\partial_{x} h_{32}\right)=0 \\
& \Gamma_{33}^{1}=\frac{1}{2} h^{11}\left(\partial_{z} h_{13}+\partial_{z} h_{31}-\partial_{x} h_{33}\right)=-\frac{\phi_{x}}{2 \phi} \\
& \Gamma_{34}^{1}=\frac{1}{2} h^{11}\left(\partial_{z} h_{14}+\partial_{I} h_{31}-\partial_{x} h_{34}\right)=0 \\
& \Gamma_{41}^{1}=\frac{1}{2} h^{11}\left(\partial_{I} h_{11}+\partial_{x} h_{41}-\partial_{x} h_{41}\right)=\frac{\phi_{z}}{2 \phi} \\
& \Gamma_{42}^{1}=\frac{1}{2} h^{11}\left(\partial_{I} h_{12}+\partial_{y} h_{41}-\partial_{x} h_{42}\right)=0 \\
& \Gamma_{43}^{1}=\frac{1}{2} h^{11}\left(\partial_{I} h_{13}+\partial_{z} h_{41}-\partial_{x} h_{43}\right)=0 \\
& \Gamma_{44}^{1}=\frac{1}{2} h^{11}\left(\partial_{I} h_{14}+\partial_{I} h_{41}-\partial_{x} h_{44}\right)=-\frac{\beta \phi_{x}+\beta_{x} \phi}{2 \phi}
\end{aligned}
$$

thus, 


$$
\Gamma^{1}=\frac{1}{2 \phi}\left[\begin{array}{cccc}
\phi_{x} & \phi_{y} & \phi_{z} & \phi_{I} \\
\phi_{y} & -\phi_{x} & 0 & 0 \\
\phi_{z} & 0 & -\phi_{x} & 0 \\
\phi_{I} & 0 & 0 & -\left(\beta \phi_{x}+\beta_{x} \phi\right)
\end{array}\right]
$$

Similarly, we have

$$
\begin{gathered}
\Gamma^{2}=\frac{1}{2 \phi}\left[\begin{array}{cccc}
-\phi_{y} & \phi_{x} & 0 & 0 \\
\phi_{x} & \phi_{y} & \phi_{z} & \phi_{I} \\
0 & \phi_{z} & -\phi_{y} & 0 \\
0 & \phi_{I} & 0 & -\left(\beta \phi_{y}+\beta_{y} \phi\right)
\end{array}\right] \\
\Gamma^{3}=\frac{1}{2 \phi}\left[\begin{array}{cccc}
-\phi_{z} & 0 & \phi_{x} & 0 \\
0 & -\phi_{z} & \phi_{y} & 0 \\
\phi_{x} & \phi_{y} & \phi_{z} & \phi_{I} \\
0 & 0 & \phi_{I} & -\left(\beta \phi_{z}+\beta_{z} \phi\right)
\end{array}\right]
\end{gathered}
$$

$\Gamma^{4}$ has a slightly different form:

$$
\begin{aligned}
& \Gamma_{11}^{4}=\frac{1}{2} h^{44}\left(\partial_{x} h_{41}+\partial_{x} h_{14}-\partial_{I} h_{11}\right)=-\frac{\phi_{I}}{2 \beta \phi} \\
& \Gamma_{12}^{4}=\frac{1}{2} h^{44}\left(\partial_{x} h_{42}+\partial_{y} h_{14}-\partial_{I} h_{12}\right)=0 \\
& \Gamma_{13}^{4}=\frac{1}{2} h^{44}\left(\partial_{x} h_{43}+\partial_{z} h_{14}-\partial_{I} h_{13}\right)=0 \\
& \Gamma_{14}^{4}=\frac{1}{2} h^{44}\left(\partial_{x} h_{44}+\partial_{I} h_{14}-\partial_{I} h_{14}\right)=\frac{\beta_{x} \phi+\beta \phi_{x}}{2 \beta \phi} \\
& \Gamma_{21}^{4}=\frac{1}{2} h^{44}\left(\partial_{y} h_{41}+\partial_{x} h_{24}-\partial_{I} h_{21}\right)=0 \\
& \Gamma_{22}^{4}=\frac{1}{2} h^{44}\left(\partial_{y} h_{42}+\partial_{y} h_{24}-\partial_{I} h_{22}\right)=-\frac{\phi_{I}}{2 \beta \phi} \\
& \Gamma_{23}^{4}=\frac{1}{2} h^{44}\left(\partial_{y} h_{43}+\partial_{z} h_{24}-\partial_{I} h_{23}\right)=0 \\
& \Gamma_{24}^{4}=\frac{1}{2} h^{44}\left(\partial_{y} h_{44}+\partial_{I} h_{24}-\partial_{I} h_{24}\right)=\frac{\beta_{y} \phi+\beta \phi_{y}}{2 \beta \phi} \\
& \Gamma_{31}^{4}=\frac{1}{2} h^{44}\left(\partial_{z} h_{41}+\partial_{x} h_{34}-\partial_{I} h_{31}\right)=0
\end{aligned}
$$




$$
\begin{aligned}
& \Gamma_{32}^{4}=\frac{1}{2} h^{44}\left(\partial_{z} h_{42}+\partial_{y} h_{34}-\partial_{I} h_{32}\right)=0 \\
& \Gamma_{33}^{4}=\frac{1}{2} h^{44}\left(\partial_{z} h_{43}+\partial_{z} h_{34}-\partial_{I} h_{33}\right)=-\frac{\phi_{I}}{2 \beta \phi} \\
& \Gamma_{34}^{4}=\frac{1}{2} h^{44}\left(\partial_{z} h_{44}+\partial_{I} h_{34}-\partial_{I} h_{34}\right)=\frac{\beta_{z} \phi+\beta \phi_{z}}{2 \beta \phi} \\
& \Gamma_{41}^{4}=\frac{1}{2} h^{44}\left(\partial_{I} h_{41}+\partial_{x} h_{44}-\partial_{I} h_{41}\right)=\frac{\beta_{x} \phi+\beta \phi_{x}}{2 \beta \phi} \\
& \Gamma_{42}^{4}=\frac{1}{2} h^{44}\left(\partial_{I} h_{42}+\partial_{y} h_{44}-\partial_{I} h_{42}\right)=\frac{\beta_{y} \phi+\beta \phi_{y}}{2 \beta \phi} \\
& \Gamma_{43}^{4}=\frac{1}{2} h^{44}\left(\partial_{I} h_{43}+\partial_{z} h_{44}-\partial_{I} h_{43}\right)=\frac{\beta_{z} \phi+\beta \phi_{z}}{2 \beta \phi} \\
& \Gamma_{44}^{4}=\frac{1}{2} h^{44}\left(\partial_{I} h_{44}+\partial_{I} h_{44}-\partial_{I} h_{44}\right)=-\frac{\beta \phi_{I}}{2 \beta \phi}
\end{aligned}
$$

Thus, we have

$$
\Gamma^{4}=\frac{1}{2 \beta \phi}\left[\begin{array}{cccc}
-\phi_{I} & 0 & 0 & \beta \phi_{x}+\beta_{x} \phi \\
0 & -\phi_{I} & 0 & \beta \phi_{y}+\beta_{y} \phi \\
0 & 0 & -\phi_{I} & \beta \phi_{z}+\beta_{z} \phi \\
\beta \phi_{x}+\beta_{x} \phi & \beta \phi_{y}+\beta_{y} \phi & \beta \phi_{z}+\beta_{z} \phi & \beta \phi_{I}
\end{array}\right] .
$$

Now we get back to Equation A.8:

$$
\frac{\partial X^{1}}{\partial t}=\frac{\phi^{2}}{\sqrt{g}} \partial_{\mu}\left(\sqrt{g} g^{\mu \nu} \partial_{\nu} X^{1}\right)+\phi^{2} \Gamma_{j k}^{1} \partial_{\mu} X^{j} \partial_{\nu} X^{k} g^{\mu \nu},
$$

We derive the two righthand terms in Equation A.14 separately:

$$
\begin{aligned}
-S_{11} & =\frac{\phi^{2}}{\sqrt{g}} \partial_{\mu}\left(\sqrt{g} g^{\mu \nu} \partial_{\nu} X^{1}\right) \\
& =\frac{\phi^{2}}{\sqrt{g}}\left[\partial_{x}\left(\sqrt{g} g^{11}\right)+\partial_{y}\left(\sqrt{g} g^{21}\right)+\partial_{z}\left(\sqrt{g} g^{31}\right)\right] \\
& =\frac{\phi^{2}}{\sqrt{g}}\left\{\partial_{x} \frac{\sqrt{\phi}\left(1+\beta I_{y}^{2}+\beta I_{z}^{2}\right)}{\sqrt{1+\beta I_{x}^{2}+\beta I_{y}^{2}+\beta I_{z}^{2}}}-\partial_{y} \frac{\sqrt{\phi} \beta I_{x} I_{y}}{\sqrt{1+\beta I_{x}^{2}+\beta I_{y}^{2}+\beta I_{z}^{2}}}-\partial_{z} \frac{\sqrt{\phi} \beta I_{x} I_{z}}{\sqrt{1+\beta I_{x}^{2}+\beta I_{y}^{2}+\beta I_{z}^{2}}}\right\}
\end{aligned}
$$




$$
\begin{aligned}
& \frac{\partial}{\partial x}\left[\frac{\phi\left(1+\beta I_{y}^{2}+\beta I_{z}^{2}\right)}{\sqrt{\phi\left(1+\beta I_{x}^{2}+\beta I_{y}^{2}+\beta I_{z}^{2}\right)}}\right] \\
& =\frac{1}{2\left[\phi\left(1+\beta I_{x}^{2}+\beta I_{y}^{2}+\beta I_{z}^{2}\right)\right]^{3 / 2}}\left[\phi \phi_{x}\left(1+\beta I_{y}^{2}+\beta I_{z}^{2}\right)\left(1+\beta I_{x}^{2}+\beta I_{y}^{2}+\beta I_{z}^{2}\right)\right. \\
& +2 \phi^{2}\left(\beta_{x} I_{y}^{2}+2 \beta I_{y} I_{x y}+\beta_{x} I_{z}^{2}+2 \beta I_{z} I_{x z}\right)\left(1+\beta I_{x}^{2}+\beta I_{y}^{2}+\beta I_{z}^{2}\right) \\
& \left.-\phi^{2}\left(\beta_{x} I_{x}^{2}+2 \beta I_{x} I_{x x}+\beta_{x} I_{y}^{2}+2 \beta I_{y} I_{x y}+\beta_{x} I_{z}^{2}+2 \beta I_{z} I_{x z}\right)\left(1+\beta I_{y}^{2}+\beta I_{z}^{2}\right)\right] \\
& -\frac{\partial}{\partial y}\left[\frac{\phi \beta I_{x} I_{y}}{\sqrt{\phi\left(1+\beta I_{x}^{2}+\beta I_{y}^{2}+\beta I_{z}^{2}\right)}}\right] \\
& =\frac{-1}{2\left[\phi\left(1+\beta I_{x}^{2}+\beta I_{y}^{2}+\beta I_{z}^{2}\right)\right]^{3 / 2}}\left[\phi \phi_{y} \beta I_{x} I_{y}\left(1+\beta I_{x}^{2}+\beta I_{y}^{2}+\beta I_{z}^{2}\right)\right. \\
& +2 \phi^{2}\left(\beta_{y} I_{x} I_{y}+\beta I_{y} I_{x y}+\beta I_{x} I_{y y}\right)\left(1+\beta I_{x}^{2}+\beta I_{y}^{2}+\beta I_{z}^{2}\right) \\
& \left.-\phi^{2} \beta I_{x} I_{y}\left(\beta_{y} I_{x}^{2}+2 \beta I_{x} I_{x y}+\beta_{y} I_{y}^{2}+2 \beta I_{y} I_{y y}+\beta_{y} I_{z}^{2}+2 \beta I_{z} I_{y z}\right)\right] \\
& -\frac{\partial}{\partial z}\left[\frac{\phi \beta I_{x} I_{z}}{\sqrt{\phi\left(1+\beta I_{x}^{2}+\beta I_{y}^{2}+\beta I_{z}^{2}\right)}}\right] \\
& =\frac{-1}{2\left[\phi\left(1+\beta I_{x}^{2}+\beta I_{y}^{2}+\beta I_{z}^{2}\right)\right]^{3 / 2}}\left[\phi \phi_{z} \beta I_{x} I_{z}\left(1+\beta I_{x}^{2}+\beta I_{y}^{2}+\beta I_{z}^{2}\right)\right. \\
& +2 \phi^{2}\left(\beta_{z} I_{x} I_{z}+\beta I_{z} I_{x z}+\beta I_{x} I_{z z}\right)\left(1+\beta I_{x}^{2}+\beta I_{y}^{2}+\beta I_{z}^{2}\right) \\
& \left.-\phi^{2} \beta I_{x} I_{z}\left(\beta_{z} I_{x}^{2}+2 \beta I_{x} I_{x z}+\beta_{z} I_{y}^{2}+2 \beta I_{y} I_{y z}+\beta_{z} I_{z}^{2}+2 \beta I_{z} I_{z z}\right)\right]
\end{aligned}
$$

Based on A.15, A.16, A.17, we get

$$
\begin{aligned}
-S_{11}= & \frac{\phi^{2}}{\sqrt{g}} \partial_{\mu}\left(\sqrt{g} g^{\mu \nu} \partial_{\nu} X^{1}\right) \\
= & \frac{-\phi_{x}\left(1+\beta I_{x}^{2}+\beta I_{y}^{2}+\beta I_{z}^{2}\right)+I_{x}\left(\phi_{x} \beta I_{x}+\phi_{y} \beta I_{y}+\phi_{z} \beta I_{z}-\phi_{I}\right)+\phi_{I} I_{x}}{2\left(1+\beta I_{x}^{2}+\beta I_{y}^{2}+\beta I_{z}^{2}\right)} \\
& +\frac{1}{2 \phi\left(1+\beta I_{x}^{2}+\beta I_{y}^{2}+\beta I_{z}^{2}\right)^{2}}\left[-2 \beta I_{x} I_{x x}\left(1+\beta I_{y}^{2}+\beta I_{z}^{2}\right)-2 \beta I_{x} I_{y y}\left(1+\beta I_{x}^{2}+\beta I_{z}^{2}\right)\right. \\
& -2 \beta I_{x} I_{z z}\left(1+\beta I_{x}^{2}+\beta I_{y}^{2}\right)+4 \beta^{2} I_{x}^{2} I_{y} I_{x y}+4 \beta^{2} I_{x}^{2} I_{z} I_{x z} 4 \beta^{2} I_{x} I_{y} I_{z} I_{y z} \\
& \left.-\left(I_{x}^{2}+I_{y}^{2}+I_{z}^{2}\right)\left(\beta \beta_{y} I_{x} I_{y}+\beta \beta_{z} I_{x} I_{z}-\beta_{x}-\beta \beta_{x} I_{y}^{2}-\beta \beta_{x} I_{z}^{2}\right)\right]
\end{aligned}
$$


and,

$$
\begin{aligned}
-S_{12}= & \phi^{2} \Gamma_{j k}^{1} \partial_{\mu} X^{j} \partial_{\nu} X^{k} g^{\mu \nu} \\
= & \phi^{2}\left(\Gamma_{11}^{1} g^{11}+2 \Gamma_{12}^{1} g^{12}+2 \Gamma_{13}^{1} g^{13}+2 \Gamma_{14}^{1}\left(I_{x} g^{11}+I_{y} g^{12}+I_{z} g^{13}+\Gamma_{22}^{1} g^{22}+\Gamma_{33}^{1} g^{33}\right)\right. \\
& \left.+\Gamma_{44}^{1}\left(I_{x}^{2} g^{11}+I_{y}^{2} g^{22}+I_{z}^{2} g^{33}+2 I_{x} I_{y} g^{21}+2 I_{x} I_{z} g^{31}+2 I_{y} I_{z} g^{23}\right)\right) \\
= & \frac{1}{2\left(1+\beta I_{x}^{2}+\beta I_{y}^{2}+\beta I_{z}^{2}\right)}\left[2 I_{x}\left(\phi_{x} \beta I_{x}+\phi_{y} \beta I_{y}+\phi_{z} \beta I_{z}-\phi_{I}\right)+\phi_{x}\left(1+\beta I_{x}^{2}+\beta I_{y}^{2}+\beta I_{z}^{2}\right)\right. \\
& \left.+\phi \beta_{x}\left(I_{x}^{2}+I_{y}^{2}+I_{z}^{2}\right)\right]
\end{aligned}
$$

To summarize,

$$
\begin{aligned}
\frac{\partial X^{1}}{\partial t}= & -S_{11}-S_{12} \\
= & -\frac{\phi \beta I_{x}\left[\left(1+\beta I_{y}^{2}+\beta I_{z}^{2}\right) I_{x x}+\left(1+\beta I_{x}^{2}+\beta I_{z}^{2}\right) I_{y y}+\left(1+\beta I_{x}^{2}+\beta I_{y}^{2}\right) I_{z z}\right]}{\left(\beta I_{x}^{2}+\beta I_{y}^{2}+\beta I_{z}^{2}+1\right)^{2}} \\
& +\frac{2 \phi \beta^{2} I_{x}\left(I_{x} I_{y} I_{x y}+I_{x} I_{z} I_{x z}+I_{y} I_{z} I_{y z}\right)}{\left(\beta I_{x}^{2}+\beta I_{y}^{2}+\beta I_{z}^{2}+1\right)^{2}} \\
& -\frac{3 I_{x}\left(\phi_{x} \beta I_{x}+\phi_{y} \beta I_{y}+\phi_{z} \beta I_{z}-\phi_{I}\right)-2 \phi_{I} I_{x}}{2\left(1+\beta I_{x}^{2}+\beta I_{y}^{2}+\beta I_{z}^{2}\right)} \\
& -\frac{\phi I_{x}\left(2+\beta I_{x}^{2}+\beta I_{y}^{2}+\beta I_{z}^{2}\right)\left(\beta_{x} I_{x}+\beta_{y} I_{y}+\beta_{z} I_{z}\right)}{2\left(1+\beta I_{x}^{2}+\beta I_{y}^{2}+\beta I_{z}^{2}\right)^{2}}
\end{aligned}
$$

Similarly, we have

$$
\begin{aligned}
\frac{\partial X^{2}}{\partial t}= & -S_{21}-S_{22} \\
= & -\frac{\phi \beta I_{y}\left[\left(1+\beta I_{y}^{2}+\beta I_{z}^{2}\right) I_{x x}+\left(1+\beta I_{x}^{2}+\beta I_{z}^{2}\right) I_{y y}+\left(1+\beta I_{x}^{2}+\beta I_{y}^{2}\right) I_{z z}\right]}{\left(\beta I_{x}^{2}+\beta I_{y}^{2}+\beta I_{z}^{2}+1\right)^{2}} \\
& +\frac{2 \phi \beta^{2} I_{y}\left(I_{x} I_{y} I_{x y}+I_{x} I_{z} I_{x z}+I_{y} I_{z} I_{y z}\right)}{\left(\beta I_{x}^{2}+\beta I_{y}^{2}+\beta I_{z}^{2}+1\right)^{2}} \\
& -\frac{3 I_{y}\left(\phi_{x} \beta I_{x}+\phi_{y} \beta I_{y}+\phi_{z} \beta I_{z}-\phi_{I}\right)-2 \phi_{I} I_{y}}{2\left(1+\beta I_{x}^{2}+\beta I_{y}^{2}+\beta I_{z}^{2}\right)} \\
& -\frac{\phi I_{y}\left(2+\beta I_{x}^{2}+\beta I_{y}^{2}+\beta I_{z}^{2}\right)\left(\beta_{x} I_{x}+\beta_{y} I_{y}+\beta_{z} I_{z}\right)}{2\left(1+\beta I_{x}^{2}+\beta I_{y}^{2}+\beta I_{z}^{2}\right)^{2}}
\end{aligned}
$$




$$
\begin{aligned}
\frac{\partial X^{3}}{\partial t}= & -S_{31}-S_{32} \\
= & -\frac{\phi \beta I_{z}\left[\left(1+\beta I_{y}^{2}+\beta I_{z}^{2}\right) I_{x x}+\left(1+\beta I_{x}^{2}+\beta I_{z}^{2}\right) I_{y y}+\left(1+\beta I_{x}^{2}+\beta I_{y}^{2}\right) I_{z z}\right]}{\left(\beta I_{x}^{2}+\beta I_{y}^{2}+\beta I_{z}^{2}+1\right)^{2}} \\
& +\frac{2 \phi \beta^{2} I_{z}\left(I_{x} I_{y} I_{x y}+I_{x} I_{z} I_{x z}+I_{y} I_{z} I_{y z}\right)}{\left(\beta I_{x}^{2}+\beta I_{y}^{2}+\beta I_{z}^{2}+1\right)^{2}} \\
& -\frac{3 I_{z}\left(\phi_{x} \beta I_{x}+\phi_{y} \beta I_{y}+\phi_{z} \beta I_{z}-\phi_{I}\right)-2 \phi_{I} I_{z}}{2\left(1+\beta I_{x}^{2}+\beta I_{y}^{2}+\beta I_{z}^{2}\right)} \\
& -\frac{\phi I_{z}\left(2+\beta I_{x}^{2}+\beta I_{y}^{2}+\beta I_{z}^{2}\right)\left(\beta_{x} I_{x}+\beta_{y} I_{y}+\beta_{z} I_{z}\right)}{2\left(1+\beta I_{x}^{2}+\beta I_{y}^{2}+\beta I_{z}^{2}\right)^{2}}
\end{aligned}
$$

Now for the last term,

$$
\frac{\partial X^{4}}{\partial t}=\frac{\phi^{2}}{\sqrt{g}} \partial_{\mu}\left(\sqrt{g} g^{\mu \nu} \partial_{\nu} X^{4}\right)+\phi^{2} \Gamma_{j k}^{4} \partial_{\mu} X^{j} \partial_{\nu} X^{k} g^{\mu \nu}=S_{41}+S_{42}
$$

where

$$
\begin{aligned}
S_{41}= & \frac{\phi^{2}}{\sqrt{g}} \partial_{\mu}\left(\sqrt{g} g^{\mu \nu} \partial_{\nu} X^{4}\right) \\
= & \frac{\phi^{2}}{\sqrt{g}}\left[\partial_{x}\left(\sqrt{g}\left(g^{11} I_{x}+g^{12} I_{y}+g^{13} I_{z}\right)\right)+\partial_{y}\left(\sqrt{g}\left(g^{21} I_{x}+g^{22} I_{y}+g^{23} I_{z}\right)\right)\right. \\
& \left.+\partial_{z}\left(\sqrt{g}\left(g^{31} I_{x}+g^{32} I_{y}+g^{33} I_{z}\right)\right)\right]
\end{aligned}
$$

For convenience, we divide $S_{41}$ into 3 terms:

$$
\begin{aligned}
S_{41} & =\text { term } 1+\text { term } 2+\text { term } 3 \\
\text { term } 1 & =\frac{\phi^{2}}{\sqrt{g}}\left(\partial_{x} \sqrt{g} g^{11} I_{x}+\partial_{y} \sqrt{g} g^{21} I_{x}+\partial_{z} \sqrt{g} g^{31} I_{x}\right) \\
\text { term } 2 & =\frac{\phi^{2}}{\sqrt{g}}\left(\partial_{x} \sqrt{g} g^{12} I_{x}+\partial_{y} \sqrt{g} g^{22} I_{x}+\partial_{z} \sqrt{g} g^{32} I_{x}\right) \\
\operatorname{term} 3 & =\frac{\phi^{2}}{\sqrt{g}}\left(\partial_{x} \sqrt{g} g^{13} I_{x}+\partial_{y} \sqrt{g} g^{23} I_{x}+\partial_{z} \sqrt{g} g^{33} I_{x}\right)
\end{aligned}
$$


Now we look at each of the three terms:

$$
\begin{aligned}
\operatorname{term} 1 & =\frac{\phi^{2}}{\sqrt{g}}\left\{\partial_{x} \frac{\sqrt{\phi} I_{x}\left(1+\beta I_{y}^{2}+\beta I_{z}^{2}\right)}{\sqrt{1+\beta I_{x}^{2}+\beta I_{y}^{2}+\beta I_{z}^{2}}}-\partial_{y} \frac{\sqrt{\phi} \beta I_{x}^{2} I_{y}}{\sqrt{1+\beta I_{x}^{2}+\beta I_{y}^{2}+\beta I_{z}^{2}}}-\partial_{z} \frac{\sqrt{\phi} \beta I_{x}^{2} I_{z}}{\sqrt{1+\beta I_{x}^{2}+\beta I_{y}^{2}+\beta I_{z}^{2}}}\right\} \\
& =-I_{x} S_{11}+R 1
\end{aligned}
$$

where

$$
\begin{aligned}
R_{1}=\frac{1}{2 \phi\left(1+\beta I_{x}^{2}+\beta I_{y}^{2}+\beta I_{z}^{2}\right)^{2}} & {\left[2 \phi^{2}\left(1+\beta I_{x}^{2}+\beta I_{y}^{2}+\beta I_{z}^{2}\right)\left(1+\beta I_{y}^{2}+\beta I_{z}^{2}\right) I_{x x}\right.} \\
- & 2 \phi^{2} I_{x} I_{y} I_{x} y \beta\left(1+\beta I_{x}^{2}+\beta I_{y}^{2}+\beta I_{z}^{2}\right) \\
- & \left.2 \phi^{2} I_{x} I_{z} I_{x z} \beta\left(1+\beta I_{x}^{2}+\beta I_{y}^{2}+\beta I_{z}^{2}\right)\right]
\end{aligned}
$$

similarily:

$$
\begin{aligned}
\operatorname{term} 2= & -I_{y} S_{21}+R_{2} \\
R_{2}= & \frac{1}{2 \phi\left(1+\beta I_{x}^{2}+\beta I_{y}^{2}+\beta I_{z}^{2}\right)^{2}}\left[2 \phi^{2}\left(1+\beta I_{x}^{2}+\beta I_{y}^{2}+\beta I_{z}^{2}\right)\left(1+\beta I_{y}^{2}+\beta I_{z}^{2}\right) I_{y y}\right. \\
& -2 \phi^{2} I_{x} I_{y} I_{x} y \beta\left(1+\beta I_{x}^{2}+\beta I_{y}^{2}+\beta I_{z}^{2}\right) \\
& \left.-2 \phi^{2} I_{y} I_{z} I_{y z} \beta\left(1+\beta I_{x}^{2}+\beta I_{y}^{2}+\beta I_{z}^{2}\right)\right] \\
\operatorname{term} 3= & -I_{z} S_{31}+R_{3} \\
R_{3}= & \frac{1}{2 \phi\left(1+\beta I_{x}^{2}+\beta I_{y}^{2}+\beta I_{z}^{2}\right)^{2}}\left[2 \phi^{2}\left(1+\beta I_{x}^{2}+\beta I_{y}^{2}+\beta I_{z}^{2}\right)\left(1+\beta I_{y}^{2}+\beta I_{z}^{2}\right) I_{z z}\right. \\
& -2 \phi^{2} I_{x} I_{z} I_{x} z \beta\left(1+\beta I_{x}^{2}+\beta I_{y}^{2}+\beta I_{z}^{2}\right) \\
& \left.-2 \phi^{2} I_{y} I_{z} I_{y z} \beta\left(1+\beta I_{x}^{2}+\beta I_{y}^{2}+\beta I_{z}^{2}\right)\right]
\end{aligned}
$$

To summarize: 


$$
\begin{aligned}
S_{41} & =\operatorname{term} 1+\operatorname{term} 2+\operatorname{term} 3 \\
& =-I_{x} S_{11}-I_{y} S_{21}-I_{z} S_{31}+R_{1}+R_{2}+R_{3} \\
& =\frac{1}{2 \beta\left(1+\beta I_{x}^{2}+\beta I_{y}^{2}+\beta I_{z}^{2}\right)}\left(\phi_{x} \beta I_{x}+\phi_{y} \beta I_{y}+\phi_{z} \beta I_{z}\right) \\
& -\frac{\phi\left[\left(1+\beta I_{y}^{2}+\beta I_{z}^{2}\right) I_{x x}+\left(1+\beta I_{x}^{2}+\beta I_{z}^{2}\right) I_{y y}+\left(1+\beta I_{x}^{2}+\beta I_{y}^{2}\right) I_{z z}-2 \beta I_{x}\left(I_{x} I_{y} I_{x y}+I_{x} I_{z} I_{x z}+I_{y} I_{z} I_{y z}\right)\right]}{\left(1+\beta I_{x}^{2}+\beta I_{y}^{2}+\beta I_{z}^{2}\right)^{2}} \\
& -\frac{\phi}{2\left(1+\beta I_{x}^{2}+\beta I_{y}^{2}+\beta I_{z}^{2}\right)}\left[\left(I_{x}^{2}+I_{y}^{2}+I_{z}^{2}\right)\left(\beta_{x} I_{x}+\beta_{y} I_{y}+\beta_{z} I_{z}\right)\right]
\end{aligned}
$$

Then,

$$
\begin{aligned}
S_{42}= & \phi^{2} \Gamma_{j k}^{4} \partial_{\mu} X^{j} \partial_{\nu} X^{k} g^{\mu \nu} \\
= & \Gamma_{11}^{4} g^{11}+2 \Gamma_{14}^{4}\left(I_{x} g^{11}+I_{y} g^{12}+I_{z} g^{13}\right)+2 \Gamma_{24}^{4}\left(I_{x} g^{21}+I_{y} g^{22}+I_{z} g^{23}\right) \\
& +2 \Gamma_{34}^{4}\left(I_{x} g^{31}+I_{y} g^{32}+I_{z} g^{33}\right)+\Gamma_{22}^{4} g^{22}+\Gamma_{33}^{4} g^{33} \\
& +\Gamma_{44}^{4}\left(I_{x}^{2} g^{11}+I_{y}^{2} g^{22}+I_{z}^{2} g^{33}+I_{x} I_{y} g^{12}+I_{x} I_{z} g^{13}+I_{y} I_{z} g^{23}\right) \\
= & \frac{1}{2 \beta\left(1+\beta I_{x}^{2}+\beta I_{y}^{2}+\beta I_{z}^{2}\right)}\left[-\phi_{I}\left(3+2 \beta I_{x}^{2}+2 \beta I_{y}^{2}+2 \beta I_{z}^{2}\right)\right. \\
& +2 I_{x}\left(\beta \phi_{x}+\beta_{x} \phi\right)+2 I_{y}\left(\beta \phi_{y}+\beta_{y} \phi\right)+2 I_{z}\left(\beta \phi_{z}+\beta_{z} \phi\right) \\
& \left.+\beta \phi_{I}\left(I_{x}^{2}+I_{y}^{2}+I_{z}^{2}\right)\right]
\end{aligned}
$$

So, overall,

$$
\begin{aligned}
\frac{\partial X^{4}}{\partial t}= & S_{41}+S_{42} \\
= & \frac{\phi\left[\left(1+\beta I_{y}^{2}+\beta I_{z}^{2}\right) I_{x x}+\left(1+\beta I_{x}^{2}+\beta I_{z}^{2}\right) I_{y y}+\left(1+\beta I_{x}^{2}+\beta I_{y}^{2}\right) I_{z z}-2 \beta I_{x}\left(I_{x} I_{y} I_{x y}+I_{x} I_{z} I_{x z}+I_{y} I_{z} I_{y z}\right)\right]}{\left(1+\beta I_{x}^{2}+\beta I_{y}^{2}+\beta I_{z}^{2}\right)^{2}} \\
& +\frac{3\left(\phi_{x} \beta I_{x}+\phi_{y} \beta I_{y}+\phi_{z} \beta I_{z}-\phi_{I}\right)-\phi_{I}\left(\beta I_{x}^{2}+\beta I_{y}^{2}+\beta I_{z}^{2}\right)}{2 \beta\left(1+\beta I_{x}^{2}+\beta I_{y}^{2}+\beta I_{z}^{2}\right)} \\
& +\frac{\phi\left(\beta_{x} I_{x}+\beta_{y} I_{y}+\beta_{z} I_{z}\right)\left(2+\beta I_{x}^{2}+\beta I_{y}^{2}+\beta I_{z}^{2}\right)}{2 \beta\left(1+\beta I_{x}^{2}+\beta I_{y}^{2}+\beta I_{z}^{2}\right)^{2}}
\end{aligned}
$$

We immediately notice that Equation A.20, A.21, A.22, A.25 have the same shape, and thus the variation of the surface can be written in vector form: 


$$
\frac{\partial X}{\partial t}=S_{1}\left[\begin{array}{c}
-\beta I_{x} \\
-\beta I_{y} \\
-\beta I_{z} \\
1
\end{array}\right]+S_{2}\left[\begin{array}{c}
I_{x} \\
I_{y} \\
I_{z} \\
I_{x}^{2}+I_{y}^{2}+I_{z}^{2}
\end{array}\right]+S_{3}\left[\begin{array}{c}
-\beta I_{x} \\
-\beta I_{y} \\
-\beta I_{z} \\
1
\end{array}\right]
$$

where

$$
\begin{aligned}
S_{1}= & \frac{\left.\phi\left(1+\beta I_{y}^{2}+\beta I_{z}^{2}\right) I_{x x}+\left(1+\beta I_{x}^{2}+\beta I_{z}^{2}\right) I_{y y}+\left(1+\beta I_{x}^{2}+\beta I_{y}^{2}\right) I_{z z}-2 \beta\left(I_{x} I_{y} I_{x y}+I_{x} I_{z} I_{x z}+I_{y} I_{z} I_{y z}\right)\right]}{\left(\beta I_{x}^{2}+\beta I_{y}^{2}+\beta I_{z}^{2}+1\right)^{2}} \\
& +\frac{3\left(\phi_{x} \beta I_{x}+\phi_{y} \beta I_{y}+\phi_{z} \beta I_{z}-\phi_{I}\right)}{2\left(1+\beta I_{x}^{2}+\beta I_{y}^{2}+\beta I_{z}^{2}\right)} \\
S_{2}= & -\frac{\phi_{I}}{2\left(1+\beta I_{x}^{2}+\beta I_{y}^{2}+\beta I_{z}^{2}\right)} \\
S_{3}= & -\frac{\phi\left(\beta_{x} I_{x}+\beta_{y} I_{y}+\beta z I_{z}\right)\left(2+\beta I_{x}^{2}+\beta I_{y}^{2}+\beta I_{z}^{2}\right)}{2 \beta\left(1+\beta I_{x}^{2}+\beta I_{y}^{2}+\beta I_{z}^{2}\right)}
\end{aligned}
$$

Note that in Equation A.26 we intentionally put all the terms that relate to the partial derivatives of $\beta$ in the last term $S_{3}$. 


\section{Appendix B}

\section{Impact of $\beta$}

The value of $\beta$ decides the embedded feature-space.

$$
\begin{aligned}
\frac{\partial X^{1}}{\partial t}= & -\frac{\phi \beta I_{x}\left[\left(1+\beta I_{y}^{2}+\beta I_{z}^{2}\right) I_{x x}+\left(1+\beta I_{x}^{2}+\beta I_{z}^{2}\right) I_{y y}+\left(1+\beta I_{x}^{2}+\beta I_{y}^{2}\right) I_{z z}-2 \beta\left(I_{x} I_{y} I_{x y}+I_{x} I_{z} I_{x z}+I_{y} I_{z} I_{y z}\right)\right]}{\left(1+\beta I_{x}^{2}+\beta I_{y}^{2}+\beta I_{z}^{2}\right)^{2}} \\
& -\frac{3 I_{x}\left(\phi_{x} \beta I_{x}+\phi_{y} \beta I_{y}+\phi_{z} \beta I_{z}\right)-2 \phi_{I} I_{x}}{2\left(1+\beta I_{x}^{2}+\beta I_{y}^{2}+\beta I_{z}^{2}\right)} \\
& -\frac{\phi I_{x}\left(2+\beta I_{x}^{2}+\beta I_{y}^{2}+\beta I_{z}^{2}\right)\left(\beta_{x} I_{x}+\beta_{y} I_{y}+\beta_{z} I_{z}\right)}{2\left(1+\beta I_{x}^{2}+\beta I_{y}^{2}+\beta I_{z}^{2}\right)^{2}} \\
= & -\frac{\phi I_{x}\left[\left(\frac{1}{\beta}+I_{y}^{2}+I_{z}^{2}\right) I_{x x}+\left(\frac{1}{\beta}+I_{x}^{2}+I_{z}^{2}\right) I_{y y}+\left(\frac{1}{\beta}+I_{x}^{2}+I_{y}^{2}\right) I_{z z}-2\left(I_{x} I_{y} I_{x y}+I_{x} I_{z} I_{x z}+I_{y} I_{z} I_{y z}\right)\right]}{\left(\frac{1}{\beta}+I_{x}^{2}+I_{y}^{2}+I_{z}^{2}\right)^{2}} \\
& -\frac{3 I_{x}\left(\phi_{x} I_{x}+\phi_{y} I_{y}+\phi_{z} I_{z}\right)}{2\left(\frac{1}{\beta}+I_{x}^{2}+I_{y}^{2}+I_{z}^{2}\right)}+\frac{-2 \phi_{x} I_{x}}{2 \beta\left(\frac{1}{\beta}+I_{x}^{2}+I_{y}^{2}+I_{z}^{2}\right)} \\
& -\frac{\phi I_{x}\left(\frac{2}{\beta}+I_{x}^{2}+I_{y}^{2}+I_{z}^{2}\right)\left(\beta_{x} I_{x}+\beta_{y} I_{y}+\beta_{z} I_{z}\right)}{2 \beta\left(\frac{1}{\beta}+I_{x}^{2}+I_{y}^{2}+I_{z}^{2}\right)^{2}}
\end{aligned}
$$

(when $\beta \rightarrow \infty$ )

$$
\begin{aligned}
= & -\frac{\phi I_{x}\left[\left(I_{y}^{2}+I_{z}^{2}\right) I_{x x}+\left(I_{x}^{2}+I_{z}^{2}\right) I_{y y}+\left(I_{x}^{2}+I_{y}^{2}\right) I_{z z}-2\left(I_{x} I_{y} I_{x y}+I_{x} I_{z} I_{x z}+I_{y} I_{z} I_{y z}\right)\right]}{\left(I_{x}^{2}+I_{y}^{2}+I_{z}^{2}\right)^{2}} \\
& -\frac{3 I_{x}\left(\phi_{x} I_{x}+\phi_{y} I_{y}+\phi_{z} I_{z}\right)}{2\left(I_{x}^{2}+I_{y}^{2}+I_{z}^{2}\right)}
\end{aligned}
$$

Similarly, when $\beta \rightarrow \infty$, 


$$
\begin{aligned}
\frac{\partial X^{2}}{\partial t}= & -\frac{\phi I_{y}\left[\left(I_{y}^{2}+I_{z}^{2}\right) I_{x x}+\left(I_{x}^{2}+I_{z}^{2}\right) I_{y y}+\left(I_{x}^{2}+I_{y}^{2}\right) I_{z z}-2\left(I_{x} I_{y} I_{x y}+I_{x} I_{z} I_{x z}+I_{y} I_{z} I_{y z}\right)\right]}{\left(I_{x}^{2}+I_{y}^{2}+I_{z}^{2}\right)^{2}} \\
& -\frac{3 I_{y}\left(\phi_{x} I_{x}+\phi_{y} I_{y}+\phi_{z} I_{z}\right)}{2\left(I_{x}^{2}+I_{y}^{2}+I_{z}^{2}\right)} \\
\frac{\partial X^{3}}{\partial t}= & -\frac{\phi I_{z}\left[\left(I_{y}^{2}+I_{z}^{2}\right) I_{x x}+\left(I_{x}^{2}+I_{z}^{2}\right) I_{y y}+\left(I_{x}^{2}+I_{y}^{2}\right) I_{z z}-2\left(I_{x} I_{y} I_{x y}+I_{x} I_{z} I_{x z}+I_{y} I_{z} I_{y z}\right)\right]}{\left(I_{x}^{2}+I_{y}^{2}+I_{z}^{2}\right)^{2}} \\
& -\frac{3 I_{z}\left(\phi_{x} I_{x}+\phi_{y} I_{y}+\phi_{z} I_{z}\right)}{2\left(I_{x}^{2}+I_{y}^{2}+I_{z}^{2}\right)}
\end{aligned}
$$

For the intensity deformation component,

$$
\begin{aligned}
\frac{\partial X^{4}}{\partial t}= & \frac{\phi\left[\left(1+\beta I_{y}^{2}+\beta I_{z}^{2}\right) I_{x x}+\left(1+\beta I_{x}^{2}+\beta I_{z}^{2}\right) I_{y y}+\left(1+\beta I_{x}^{2}+\beta I_{y}^{2}\right) I_{z z}-2 \beta I_{x}\left(I_{x} I_{y} I_{x y}+I_{x} I_{z} I_{x z}+I_{y} I_{z} I_{y z}\right)\right]}{\left(1+\beta I_{x}^{2}+\beta I_{y}^{2}+\beta I_{z}^{2}\right)^{2}} \\
& +\frac{3\left(\phi_{x} \beta I_{x}+\phi_{y} \beta I_{y}+\phi_{z} \beta I_{z}-\phi_{I}\right)-\phi_{I}\left(\beta I_{x}^{2}+\beta I_{y}^{2}+\beta I_{z}^{2}\right)}{2 \beta\left(1+\beta I_{x}^{2}+\beta I_{y}^{2}+\beta I_{z}^{2}\right)} \\
& +\frac{\phi\left(\beta_{x} I_{x}+\beta_{y} I_{y}+\beta_{z} I_{z}\right)\left(2+\beta I_{x}^{2}+\beta I_{y}^{2}+\beta I_{z}^{2}\right)}{2 \beta\left(1+\beta I_{x}^{2}+\beta I_{y}^{2}+\beta I_{z}^{2}\right)^{2}} \\
& =\frac{\phi\left[\left(\frac{1}{\beta}+I_{y}^{2}+I_{z}^{2}\right) I_{x x}+\left(\frac{1}{\beta}+I_{x}^{2}+I_{z}^{2}\right) I_{y y}+\left(\frac{1}{\beta}+I_{x}^{2}+I_{y}^{2}\right) I_{z z}-2 I_{x}\left(I_{x} I_{y} I_{x y}+I_{x} I_{z} I_{x z}+I_{y} I_{z} I_{y z}\right)\right]}{\beta\left(\frac{1}{\beta}+I_{x}^{2}+I_{y}^{2}+I_{z}^{2}\right)^{2}} \\
& +\frac{3\left(\phi_{x} I_{x}+\phi_{y} I_{y}+\phi_{z} I_{z}-\frac{\phi_{I}}{\beta}\right)-\phi_{I}\left(I_{x}^{2}+I_{y}^{2}+I_{z}^{2}\right)}{2 \beta\left(\frac{1}{\beta}+\beta I_{x}^{2}+\beta I_{y}^{2}+\beta I_{z}^{2}\right)} \\
+ & \frac{\phi\left(\beta_{x} I_{x}+\beta_{y} I_{y}+\beta_{z} I_{z}\right)\left(\frac{2}{\beta}+\beta I_{x}^{2}+\beta I_{y}^{2}+\beta I_{z}^{2}\right)}{2 \beta\left(\frac{1}{\beta}+\beta I_{x}^{2}+\beta I_{y}^{2}+\beta I_{z}^{2}\right)^{2}}
\end{aligned}
$$

(when $\beta \rightarrow \infty$ )

$$
=0
$$

These results show that when $\beta$ is set to a very large value, the spatial grid deformation will dominate. Now, we test the case when $\beta \rightarrow 0$ : 


$$
\begin{aligned}
\frac{\partial X^{1}}{\partial t}= & -\frac{\phi \beta I_{x}\left[\left(1+\beta I_{y}^{2}+\beta I_{z}^{2}\right) I_{x x}+\left(1+\beta I_{x}^{2}+\beta I_{z}^{2}\right) I_{y y}+\left(1+\beta I_{x}^{2}+\beta I_{y}^{2}\right) I_{z z}-2 \beta\left(I_{x} I_{y} I_{x y}+I_{x} I_{z} I_{x z}+I_{y} I_{z} I_{y z}\right)\right]}{\left(1+\beta I_{x}^{2}+\beta I_{y}^{2}+\beta I_{z}^{2}\right)^{2}} \\
& -\frac{3 I_{x}\left(\phi_{x} \beta I_{x}+\phi_{y} \beta I_{y}+\phi_{z} \beta I_{z}\right)-2 \phi_{I} I_{x}}{2\left(1+\beta I_{x}^{2}+\beta I_{y}^{2}+\beta I_{z}^{2}\right)} \\
& -\frac{\phi I_{x}\left(2+\beta I_{x}^{2}+\beta I_{y}^{2}+\beta I_{z}^{2}\right)\left(\beta_{x} I_{x}+\beta_{y} I_{y}+\beta_{z} I_{z}\right)}{2\left(1+\beta I_{x}^{2}+\beta I_{y}^{2}+\beta I_{z}^{2}\right)^{2}}
\end{aligned}
$$

(when $\beta \rightarrow 0$ )

$$
=\phi_{I} I_{x}-2 \phi I_{x}\left(\beta_{x} I_{x}+\beta_{y} I_{y}+\beta_{z} I_{z}\right)
$$

Similarly, when $\beta \rightarrow 0$

$$
\begin{aligned}
& \frac{\partial X^{2}}{\partial t}=\phi_{I} I_{y}-2 \phi I_{y}\left(\beta_{x} I_{x}+\beta_{y} I_{y}+\beta_{z} I_{z}\right) \\
& \frac{\partial X^{3}}{\partial t}=\phi_{I} I_{z}-2 \phi I_{z}\left(\beta_{x} I_{x}+\beta_{y} I_{y}+\beta_{z} I_{z}\right)
\end{aligned}
$$

For the intensity deformation component,

$$
\begin{aligned}
\frac{\partial X^{4}}{\partial t}= & \frac{\phi\left[\left(1+\beta I_{y}^{2}+\beta I_{z}^{2}\right) I_{x x}+\left(1+\beta I_{x}^{2}+\beta I_{z}^{2}\right) I_{y y}+\left(1+\beta I_{x}^{2}+\beta I_{y}^{2}\right) I_{z z}-2 \beta I_{x}\left(I_{x} I_{y} I_{x y}+I_{x} I_{z} I_{x z}+I_{y} I_{z} I_{y z}\right)\right]}{\left(1+\beta I_{x}^{2}+\beta I_{y}^{2}+\beta I_{z}^{2}\right)^{2}} \\
& +\frac{3\left(\phi_{x} \beta I_{x}+\phi_{y} \beta I_{y}+\phi_{z} \beta I_{z}-\phi_{I}\right)-\phi_{I}\left(\beta I_{x}^{2}+\beta I_{y}^{2}+\beta I_{z}^{2}\right)}{2 \beta\left(1+\beta I_{x}^{2}+\beta I_{y}^{2}+\beta I_{z}^{2}\right)} \\
& +\frac{\phi\left(\beta_{x} I_{x}+\beta_{y} I_{y}+\beta_{z} I_{z}\right)\left(2+\beta I_{x}^{2}+\beta I_{y}^{2}+\beta I_{z}^{2}\right)}{2 \beta\left(1+\beta I_{x}^{2}+\beta I_{y}^{2}+\beta I_{z}^{2}\right)^{2}}
\end{aligned}
$$

(when $\beta \rightarrow 0$ )

$$
\begin{aligned}
& =\phi\left(I_{x x}+I_{y y}+I_{z z}\right)-\frac{3 \phi_{I}}{0}+\frac{2 \phi\left(\beta_{x} I_{x}+\beta_{y} I_{y}+\beta_{z} I_{z}\right)}{0} \\
& =\infty
\end{aligned}
$$

These results shows that when $\beta$ is set to a very small value, the intensity deformation will dominate. 


\section{Bibliography}

[1] N. Sochen, R. Kimmel, and R. Malladi, "From high energy physics to low level vision," Technical Report 39243, UC Berkeley, 1996.

[2] X. Long and C. Wyatt, "An automatic unsupervised classification of mr images in alzheimer's disease," in IEEE Conference on Computer Vision and Pattern Recognition (CVPR), pp. 2910 - 2917, 2010.

[3] P. M. Thompson and A. W. Toga, "A framework for computational anatomy," Computing and Visualization in Science, vol. 5, no. 1, pp. 13 - 34, 2002.

[4] C. Leondes, Biomechanical systems technology, ch. 1, pp. 1-29. Singapore: World Scientific, 2007. Deformable Image Registration for Radiation Therapy Planning: Algorithms and Applications, by M.M. Kaus and K.K. Brock.

[5] A. Klein, J. Andersson, B. A. Ardekani, J. Ashburner, B. Avants, M. Chiang, G. E. Christensen, D. L. Collins, J. Gee, P. Hellier, J. H. Song, M. Jenkinson, C. Lepage, D. Rueckert, P. Thompson, T. Vercauteren, R. P. Woods, J. J. Mann, and R. V. Parsey, "Evaluation of 14 nonlinear deformation algorithms applied to human brain mri registration," NeuroImage, vol. 46, no. 3, pp. 786-802, 2009.

[6] J. V. Hajnal, D. L. Hill, and D. J. Hawkes, Medical Image Registration. CRC Press, 2001.

[7] J. Modersitzki, Numerical Methods for Image Registration. Oxford Science Publications, 2004.

[8] T. S. Yoo, Insight into Images, Principles and Practise. A K Peters, Ellesley, Massachusetts, 2004. 
[9] X. Li and C. Wyatt, "Modeling topological changes in deformable registration," in ISBI'10: Proceedings of the 2010 IEEE international conference on Biomedical imaging, pp. 360-363, 2010.

[10] M. Sdika and D. Pelletier, "Nonrigid registration of multiple sclerosis brain images using lesion inpainting for morphometry or lesion mapping," Human Brain Mapping, vol. 30(4), pp. 1060-1067, 2009.

[11] L. Ibanez, W. Schroeder, L. Ng, J. Cates, and the Insight Software Consortium, 2005. The ITK Software Guide, Second Edition, Updated for ITK version 2.4.

[12] M. F. Beg, M. I. Miller, A. Trouv, and L. Younes, "Computing large deformation metric mappings via geodesic flows of diffeomorphisms," International Journal of Computer Vision, vol. 61, pp. 139-157, February 2005.

[13] T. Vercauteren, X. Pennec, A. Perchant, and N. Ayache, "Non-parametric diffeomorphic image registration with the demons algorithm," in MICCAI Lecture Notes in Computer Science, vol. 4792, pp. 319-326, October 2007.

[14] D. Shen and C. Davatzikos, "Hammer: hierarchical attribute matching mechanism for elastic registration," IEEE Tansact. Med Imaging, vol. 21, pp. 786-802, 2002.

[15] D. Essen, H. Drury, J. Dickson, J. Harwell, D. Hanlon, and C. Anderson, "An integrated software suite for surface-based analyses of cerebral cortex," J. Am. Med.Inform. Assoc.: JAMIA, vol. 8, pp. 443-459, 2001.

[16] B. Fischl, A. van der Kouwe, C. Destrieux, E. Halgren, F. Sgonne, D. Salat, E. Busa, L. Seidman, J. Goldstein, D. Kennedy, V. Caviness, N. Makris, B. Rosen, and A. Dale, "Automatically parcellating the human cerebral cortex," Cereb. Cortex, vol. 14, pp. 11$22,2004$.

[17] A. Dale, B. Fischl, and M. I. Sereno, "Cortical surface-based analysis. i. segmentation and surface reconstruction.," Neuroimage, vol. 9, no. 2, pp. 179-194, 1999.

[18] R. S. Frackowiak, J. T. Ashburner, W. D. Penny, S. Zeki, K. J. Friston, C. D. Frith, R. J. Dolan, and C. J. Price, Human Brain Function, ch. 4. Academic Press; 2 edition, 2004. High-dimensional image warping, by J. Ashburner and K. Friston. 
[19] O. Musse, F. Heitz, and J. Armspach, "Topology preserving deformable image matching using constrained hierarchical parametric models," IEEE Transactions on Image Processing, vol. 10, pp. 1081 - 1093, July 2001.

[20] V. Noblet, C. Heinrich, F. Heitz, and J.-P. Armspach, "3-d deformable image registration: a topology preservation scheme based on hierarchical deformation models and interval analysis optimization," IEEE Transactions on Image Processing, vol. 14, pp. 553-566, May 2005.

[21] H. Johnson, G. Harris, and K. Williams, 2009. BRAINSFit @ http://www.nitrc.org/docman/view.php/52/639/BRAINSFit.pdf.

[22] D. T. Chard, J. S. Jackson, D. H. Miller, and C. A. Wheeler-Kingshott, "Reducing the impact of white matter lesions on automated measures of brain gray and white matter volumes," Journal of Magnetic Resonance Imaging, vol. 32(1), pp. 223-228, 2001.

[23] A. Trouve and L. Younes, "Metamorphoses through lie group action," Foundations of Computational Mathematics, vol. 5(2), 2005.

[24] T. Hartkens, D. L. G. Hill, A. D. Castellano-Smith, D. J. Hawkes, C. R. Maurer, A. J. Martin, W. A. Hall, H. Liu, and C. L. Truwit, "Using points and surfaces to improve voxel-based non-rigid registration," Lecture Notes in Computer Science, vol. 2489, pp. 565-572, 2002.

[25] P. Fletcher, S. Venkatasubramanian, and S. Joshi, "The geometric median on Riemannian manifolds with application to robust atlas estimation," NeuroImage, vol. 45, pp. 143-152, 2009.

[26] E. Zacharaki, C. Hogeaand, D. Shen, G. Biros, and C. Davatzikos, "Non-diffeomorphic registration of brain tumor images by simulating tissue loss and tumor growth," Neuroimage, vol. 46, pp. 762-774, 2009.

[27] N. Hata, T. Dohi, S. Warfield, W. M. Wells, R. Kikinis, and F. A. Jolesz, "Multimodality deformable registration of pre- and interoperate images for mri-guided brain surgery," in Medical Image Computing and Computer-Assisted Intervention-MICCAI, 1998.

[28] O. Clatz, H. Delingette, I.-F. Talos, A. Golby, R. Kikinis, F. Jolesz, N. Ayache, and S. Warfield, "Robust nonrigid registration to capture brain shift from intraoperative mri," Medical Imaging, IEEE Transactions on, vol. 24, pp. 1417 -1427, nov. 2005. 
[29] P. Risholm, E. Samset, and I. W. Wells, "Validation of a non-rigid registration framework that accommodates tissue resection," in SPIE Medical Imaging, 2010.

[30] T. Hartkens, D. Hill, A. Castellano-Smith, D. Hawkes, C. Maurer, A. Martin, W. Hall, H. Liu, and C. Truwit, "Using points and surfaces to improve voxel-based non-rigid registration," in Medical Image Computing and Computer-Assisted Intervention (MICCAI), vol. 2489 of Lecture Notes in Computer Science, pp. 565-572, 2002.

[31] S. Periaswamy and H. Farid, "Medical image registration with partial data," Medical Image Analysis, vol. 10, p. 2006, 2005.

[32] N. Chitphakdithai and J. S. Duncan, "Pairwise registration of images with missing correspondences due to resection," in ISBI'10: Proceedings of the 2010 IEEE international conference on Biomedical imaging, pp. 1025-1028, 2010.

[33] M. Cuzol, P. Worsley, and E. Memin, "A novel parametric method for non- rigid image registration," in IPMI, pp. 456-467, 2005.

[34] G. Gilboa, N. Sochen, and Y. Y. Zeevi, "Forward-and-backward diffusion processes for adaptive image enhancement and denoising," IEEE Trans. Image Process., vol. 11, pp. 689-703, 2002.

[35] R. G. Boyes, J. L. Gunter, C. Frost, A. L. Janke, T. Yeatman, D. L. G. Hill, M. A. Bernstein, P. M. Thompson, M. W. Weiner, N. Schuff, G. E. Alexander, R. J. Killiany, C. DeCarli, C. R. Jack, N. C. Fox, and A. D. N. I. Study, "Intensity non-uniformity correction using n3 on 3-t scanners with multichannel phased array coils," Neuroimage, vol. 39(4), pp. 1752-1762, 2008.

[36] A. Banyaga, The structure of classical diffeomorphism groups, Mathematics and its Applications. Kluwer Academic, 1997. ISBN 0-7923-4475-8.

[37] H. Omori, Infinite-dimensional Lie groups, Translations of Mathematical Monographs. American Mathematical Society, 1997. ISBN 0-8218-4575-6.

[38] Y. Borisovich, N. Bliznyakov, T. Fomenko, and Y. Izrailevich, Introduction to differential and algebraic topology. Kluwer Academic Publishers, 1995. ISBN 0-7923-3499-X.

[39] J.-P. Thirion, "Image matching as a diffusion process: An analogy with maxwell's demons," Medical Image Analysis, vol. 2, no. 3, pp. 243-260, 1998. 
[40] J. Pluim, J. Maintz, and M. Viergever, "Mutual information based registration of medical images: A survey," IEEE Trans on Medical Imaging, pp. 986-1004, 2003.

[41] T. Vercauteren, X. Pennec, A. Perchant, and N. Ayache, "Symmetric log-domain diffeomorphic registration: A demons-based approach," pp. 754-761, 2008.

[42] R. Mahony and J. Manton, "The geometry of the newton method on non-compact lie-groups," J. Global Optim., vol. 23, no. 3, pp. 309-327, 2002.

[43] E. Malis, "Improving vision-based control using efficient second-order minimization techniques," in Proc. ICRA, April 2004.

[44] V. Arsigny, O. Commonwick, X. Pennec, and N. Ayache, "Statistics on diffeomorphisms in a log-euclidean framework," in MICCAI, vol. 4792, pp. 924-931, 2006.

[45] M. Bossa, M. Hernandez, and S. Olmos, "Contributions to 3d diffeomorphic atlas estimation: Application to brain images," pp. 667-674, 2007.

[46] P. Dupuis, U. Grenander, and M. I. Miller, "Variational problems on flows of diffeomorphisms for image matching," Quart. Appl. Math., pp. 587-600, 1998.

[47] A. Trouve, "Diffeomorphism groups and pattern matching in image analysis," J. Comput. Vis., vol. 28, pp. 213-221, 1998.

[48] M. I. Miller, "Computational anatomy: shape, growth, and atrophy comparison via diffeomorphisms," NeuroImage, vol. 23, no. Supplement 1, pp. S19 - S33, 2004. Mathematics in Brain Imaging.

[49] M. Hernandez, M. Bossa, and S. Olmos, "Registration of anatomical images using geodesic paths of diffeomorphisms parameterized with stationary vector fields," in $M M$ $B I A, 2007$.

[50] J. Ashburner, "A fast diffeomorphic image registration algorithm," Neuroimage, vol. 38, no. 1, pp. 95-113, 2007.

[51] M. Hernandez, S. Olmos, and X. Pennec, "Comparing algorithms for diffeomorphic registration: Stationary LDDMM and diffeomorphic demons," in Proc. of the International Workshop on the Mathematical Foundations of Computational Anatomy (MFCA-2008) (X. Pennec and S. Joshi, eds.), September 2008. 
[52] D. Collins, A. Zijdenbos, V. Kollokian, J. Sled, N. Kabani, C. Holmes, and A. Evans, "Design and construction of a realistic digital brain phantom," IEEE Transactions on Medical Imaging, vol. 17, pp. 463-468, June 1998.

[53] A. Yezzi, S. Kichenassamy, A. Kumar, P. Olver, and A. Tannenbaum, "A geometric snake model for segmentation of medical imagery," IEEE Trans. on Medical Imaging, vol. 16, pp. 199-209, 1997.

[54] V. Caselles, R. Kimmel, , and G. Sapiro, "Geodesic active contours," International Journal Of Computer Vision, vol. 22, pp. 61-79.

[55] X. Long and C. Wyatt, "Structural template formation with discovery of subclasses," in Proc. SPIE 76231B, 2010.

[56] D. S. Marcus., T. H. Wang, J. Parker, J. G. Csernansky, J. Morris, and R. Buckner, "Open access series of imaging studies (oasis): Cross-sectional mri data in young, middle aged, nondemented, and demented older adults," J. Cognitive Neuroscience, vol. 19, no. 9, pp. 1498-1507, 2007.

[57] "http://www.slicer.org/."

[58] S. Smith, P. R. Bannister, C. Beckmann, J. M. Brady, S. Clare, D. Flitney, P. Hansen, M. Jenkinson, D. Leibovici, B. Ripley, M. Woolrich, and Y. Zhang, "FSL: New tools for functional and structural brain image analysis," NeuroImage, vol. 13, 2001. 